\title{
THE STABILITY OF FULL DIMENSIONAL KAM TORI FOR NONLINEAR SCHRÖDINGER EQUATION
}

\author{
HONGZI CONG, JIANJUN LIU, YUNFENG SHI, AND XIAOPING YUAN
}

\begin{abstract}
In this paper, it is proved that the full dimensional invariant tori obtained by Bourgain [J. Funct. Anal., 229 (2005), no. 1, 62-94.] is stable in a very long time for $1 \mathrm{D}$ nonlinear Schrödinger equation with periodic boundary conditions.
\end{abstract}

\section{Introduction AND MAIN RESUlts}

Consider a Hamiltonian of $n$-freedom

$$
H=H_{0}(I)+\epsilon H_{1}(\theta, I),
$$

with the standard symplectic structure $\mathrm{d} \theta \wedge \mathrm{d} I$ on $\mathbb{T}^{n} \times \mathbb{R}^{n}$ and the angle-action variable $(\theta, I)$ belongs to some domain $\mathbb{T}^{n} \times D \subseteq \mathbb{T}^{n} \times \mathbb{R}^{n}$. Assume the unperturbed Hamiltonian $H_{0}(I)$ is independent of $\theta$ and satisfies Kolmogorov non-degenerate condition

$$
\operatorname{det}\left(\partial^{2} H_{0}(I)\right) \neq 0, I \in D \text {. }
$$

Also assume $H_{0}, H_{1}$ are smooth sufficiently. Then the well-known KolmogorovArnold-Moser (KAM) theorem (30, 1, 38]) claims that any invariant tori of the unperturbed $H_{0}$ with prescribed Diophantine frequency $\omega\left(I_{0}\right)=\frac{\partial H_{0}\left(I_{0}\right)}{\partial I}$ for some $I_{0} \in D$ persist under a small perturbation $\epsilon H_{1}(I, \theta)$. This theorem is now called the classical KAM theorem and the persisted tori called full dimensional KAM tori. The huge challenge is encountered when one tries to extend the classical KAM theorem to the Hamiltonian defined by some PDEs because of very complicated resonant relationships among the infinitely many number of frequencies. In order to evade this impasse, one considers the Hamiltonian of the form

$$
H=N+\epsilon P(\theta, I, z, \bar{z}),
$$

with the symplectic structure $\mathrm{d} \theta \wedge \mathrm{d} I+\sqrt{-1} \mathrm{~d} z \wedge \mathrm{d} \bar{z}$ on $\mathbb{T}^{n} \times \mathbb{R}^{n} \times \mathcal{H} \times \mathcal{H} \ni(\theta, I, z, \bar{z})$ and

$$
N=\sum_{i=1}^{n} \omega_{i} I_{i}+\sum_{j=1}^{\tau} \Omega_{j} z_{j} \bar{z}_{j}, 1 \leq n<\infty, 1 \leq \tau \leq \infty,
$$

where $\mathcal{H}$ is a Hilbert space of dimension $\tau, \omega=\left(\omega_{1}, \omega_{2}, \cdots, \omega_{n}\right)$ called tangent frequency vector, $\left(\Omega_{j}\right)_{1 \leq j \leq \tau}$ the normal frequency vector, and $P=P(\theta, I, z, \bar{z})$ is a perturbation. The unperturbed Hamiltonian $N$ has a special invariant torus

$$
\mathcal{T}_{0}=\{\theta=\omega t\} \times\{I=0\} \times\{z=0\} \times\{\bar{z}=0\} .
$$

2000 Mathematics Subject Classification. Primary 37K55, 37J40; Secondary 35B35, 35Q35.

Key words and phrases. Stability for Hamilton PDEs; Almost periodic solution; full dimensional tori; NLS equation. 
Under suitable assumptions on $N$ and $P$, it can be proved that for "most" frequency $\omega$, the tori $\mathcal{T}_{0}$ can be persisted for some small perturbation $\epsilon P$ (see [26] when $\tau<\infty$, see [31, 34] and 44] when $\tau=\infty)$. In 34, 44, the frequency vector $\omega \in \mathbb{R}^{n}$ is regarded as parameter. In [26], the frequency

$$
\omega=\omega_{0} t,
$$

where $\omega_{0} \in \mathbb{R}^{n}$ is a fixed Diophantine vector and $t \in \mathbb{R}$ is regarded as parameter. Anyway, it is proved that a 1-dimensional parameter is needed in [13, at least, when considering the lower dimensional KAM tori. Thus the work by [26] is optimal in this sense, and the frequencies of those lower dimensional tori can not prescribed prior. Besides, the KAM theorem of this type depends heavily on the fact that the spatial dimension of the PDEs equals to 1. Bourgain 14, 18, developed a new method initialed by Craig-Wayne [22] to deal with the KAM tori for the PDEs in high spatial dimension, based on the Newton iteration, Fröhlich-Spencer techniques, Harmonic analysis and semi-algebraic set theory (see [18). This method is now called C-W-B method. We also mention [25] where the KAM theorem is extended in the direction of $34,31,26,44$ to deal with higher spatial dimensional nonlinear Schrödinger equation. In addition, the KAM theory is also developed to deal some 1-dimensional PDEs of unbounded perturbation. See, for example, 34, 40, 36, 46, 2, 3], 28, for the details. In the all above works, the obtained KAM tori are lower (finite) dimension, considering that the Hamiltonian PDEs are infinite dimensional.

Naturally, the following problem is interesting:

Can the full dimensional KAM tori be expected with a suitable decay, for example, $I_{n} \sim|n|^{-S}$ with some $S>0$ as $|n| \rightarrow+\infty$ ?

The existence of the full dimensional KAM tori with decay rate $I_{n} \sim|n|^{-S}$ is still open up to now. See 35 for the details. One way to obtain the existence of full dimensional KAM tori is to use repeatedly (infinitely many times) the KAM theorem dealing with lower dimensional KAM tori. See 43 and 12 and some other references. However, the amplitude (or action) of those full dimensional KAM tori decays extremely fast. In fact, the decay rate is defined implicitly and much more fast than $I_{n} \sim e^{-|n|^{S}}, S>1$. See more comments in [17]. Another way is due to Bourgain in [19] where 1-dimensional nonlinear Schrödinger equation with periodic boundary condition is investigated (Also see [41] given by Pöschel where infinite dimensional Hamiltonian systems with short range is considered). It is shown in 19 that 1D NLS has a full dimensional KAM torus of prescribed frequencies with the actions of the tori obeying the estimates

$$
\frac{1}{2} e^{-r|n|^{1 / 2}} \leq I_{n} \leq 2 e^{-r|n|^{1 / 2}}, n \in \mathbb{Z}, r>0 .
$$

This is up to now only one existence result about the full dimensional KAM tori with a slower decay rate than $I_{n} \sim e^{-|n|^{S}}, S>1$.

On the other hand, it is well-known that a physical quantity is observable only if it is stable at least for a long time. Naturally, one has the following question:

Are the full dimensional KAM tori obtained by Bourgain in 19] stable for a long time? 
THE STABILITY OF FULL DIMENSIONAL KAM TORI FOR NONLINEAR SCHRÖDINGER EQUATIOß

There have been a relatively long history about the long time stability for the finite dimensional Hamiltonian (1.1) of freedom $n<\infty$. If the unperturbed $H_{0}$ is convex ( the steepest, in Nekhoroshev's terminology), any solutions including the KAM tori are stable in long time $|t|<\exp \left(\epsilon^{-\frac{1}{2 n}}\right)$, by using Nekhoroshev'e estimate 39, 42. In fact, those KAM tori are stable in a much longer time $\exp \left(\exp \left(\epsilon^{-\frac{1}{a}}\right)\right)$ with some $a>n$ (see [37] for the details). Clearly, the stability of this kind can not be generalized to the Hamiltonian PDEs including NLS, in view of $n=\infty$ at this case. Bambusi [4] and Bourgain [15] initiated the study of the stability in long time $|t|<\epsilon^{-M}$ with a large $M>0$ for the equilibrium $u=0$ for some Hamiltonian PDEs including NLS. See [5, 8, 4, 6, 7, 9, 10, 11, 15, 23, 24, 27, 29, 45, for example, for more results. Recently, 20, and 21 investigated the stability in long time for the lower (finite) dimensional KAM tori for PDEs.

According to our best knowledge, there has not yet been any result with respect to the stability in long time for the full dimensional KAM tori for the Hamiltonian PDEs. The main aim of the present paper is to prove that the full dimensional KAM tori obtained by Bourgain are stable in a long time. Incidentally, we will also prove that those tori are linearly stable.

In order to state our theorem, let us begin with the nonlinear Schrödinger equation with periodic boundary conditions

$$
\mathbf{i} u_{t}-u_{x x}+M u+\epsilon|u|^{4} u=0, \quad x \in \mathbb{T},
$$

where $M$ is a random Fourier multiplier defined by

$$
\widehat{M u}(n)=V_{n} \widehat{u}(n)
$$

and $\left(V_{n}\right)_{n \in \mathbb{Z}}$ are independently chosen in $[-1,1]$. Written in Fourier modes $\left(q_{n}\right)_{n \in \mathbb{Z}}$, then (1.3) can be rewritten as

$$
\dot{q}_{n}=\mathbf{i} \frac{\partial H}{\partial \bar{q}_{n}}
$$

with the Hamiltonian

$$
H(q, \bar{q})=\sum_{n \in \mathbb{Z}}\left(n^{2}+V_{n}\right)\left|q_{n}\right|^{2}+\epsilon \sum_{n_{1}-n_{2}+n_{3}-n_{4}+n_{5}-n_{6}=0} q_{n_{1}} \bar{q}_{n_{2}} q_{n_{3}} \bar{q}_{n_{4}} q_{n_{5}} \bar{q}_{n_{6}} .
$$

Fix $0<\theta<1$ and introduce for any $r>0$ the Banach space $\mathfrak{H}_{r, \infty}$ of all complexvalued sequences $q=\left(q_{n}\right)_{n \in \mathbb{Z}}$ with

$$
\|q\|_{r, \infty}=\sup _{n \in \mathbb{Z}}\left|q_{n}\right| e^{r|n|^{\theta}}<\infty
$$

For $x \in \mathbb{R}$, denote $\|x\|=\inf _{y \in \mathbb{Z}}|x-y|$. Then we say a vector $V \in[-1,1]^{\mathbb{Z}}$ is Diophantine, if there exists a real number $\gamma>0$ such that

$$
\left\|\sum_{n \in \mathbb{Z}} l_{n} V_{n}\right\| \geq \gamma \prod_{n \in \mathbb{Z}} \frac{1}{1+l_{n}^{2}|n|^{4}},
$$

for any $l \in \mathbb{Z}^{\mathbb{Z}}$ with $0<\# \operatorname{supp} l<\infty$, where

$$
\operatorname{supp} l=\left\{n: l_{n} \neq 0\right\}
$$

and

$$
|n|=\max \{1, n,-n\} .
$$


From Lemma 4.1 in [19], we know the following resonance issue:

$$
P\left\{V:\left\|\sum_{n \in \mathbb{Z}} l_{n} V_{n}\right\|<\gamma \prod_{n \in \mathbb{Z}} \frac{1}{1+l_{n}^{2}|n|^{4}}, \forall l \neq 0 \text { with \#supp } l<\infty\right\}<C \gamma,
$$

where $P$ is the standard probability measure on $[-1,1]^{\mathbb{Z}}$ and $C>0$ is an absolute constant.

Theorem 1.1. Given $0<\theta<1, r>0$ and a Diophantine vector $\omega=\left(\omega_{n}\right)_{n \in \mathbb{Z}}$ satisfying $\sup _{n}\left|\omega_{n}\right|<1$, then for sufficiently small $\epsilon>0$ and appropriate $M$, (1.3) has a full dimensional invariant torus $\mathfrak{T}$ satisfying:

(1) the amplitude on $\mathfrak{T}$ is restricted as

$$
\frac{1}{2} e^{-r|n|^{\theta}} \leq\left|q_{n}\right| \leq e^{-r|n|^{\theta}}
$$

(2) the frequency on $\mathfrak{T}$ is prescribed to be $\left(n^{2}+\omega_{n}\right)_{n \in \mathbb{Z}}$;

(3) the invariant tori $\mathfrak{T}$ are linearly stable;

(4) the invariant tori are stable in a long time in the sense that for any small enough $\tau$ (independent of $\epsilon$ ), if

$$
d(q(0), \mathfrak{T}) \leq \tau,
$$

then

$$
d(q(t), \mathfrak{T}) \leq 2 \tau, \quad \text { for all }|t| \leq \tau^{-\frac{1}{4}|\ln \tau|^{\frac{\theta}{10}}+1},
$$

where

$$
d(q(0), \mathfrak{T})=\inf _{w \in \mathfrak{T}}\|q(0)-w\|_{r, \infty}
$$

Remark 1.2. In order to fulfill the decay rate, Bourgain introduced a weight function $\sum_{n}\left(2 a_{n}+k_{n}+k_{n}^{\prime}\right)|n|^{1 / 2}$ for a polynomial Hamiltonian

$$
\sum_{a, k, k^{\prime}} B_{a k k^{\prime}} \prod_{n} I_{n}^{a_{n}} q_{n}^{k_{n}} \bar{q}_{n}^{k_{n}^{\prime}}
$$

In Remark 2 (p. 67, [19), Bourgain stated that the weight function $\sum_{n}\left(2 a_{n}+k_{n}+\right.$ $\left.k_{n}^{\prime}\right)|n|^{1 / 2}$ may have been replaced by expression $\sum_{n}\left(2 a_{n}+k_{n}+k_{n}\right)|n|^{\theta}$ for some $0<\theta<1$. In the present paper, we fulfill the Bourgain's statement incidentally.

\section{The Norm of the Hamiltonian}

Most of the notations come from 19 for the reader's easy understanding. The analysis will be performed in complex conjugate variables $\left(q_{n}, \bar{q}_{n}\right)$ without passing to action-angle variables. The Hamiltonian expressions may involve $I_{n}=\left|q_{n}\right|^{2}$ and $J_{n}=I_{n}-I_{n}(0)$ as notations but not as new variables, where $I_{n}(0)$ will be considered as the initial data. At every stage of the iteration, the Hamiltonian $H$ will be expanded in monomials $\mathcal{M}_{a k k^{\prime}}\left(a, k, k^{\prime} \in \mathbb{N}^{\mathbb{Z}}\right.$ are multi-indices) of the following form:

$$
\mathcal{M}_{a k k^{\prime}}=\prod_{n \in \mathbb{Z}} I_{n}(0)^{a_{n}} q_{n}^{k_{n}} \bar{q}_{n}^{k_{n}^{\prime}}
$$

where

$$
\sum_{n \in \mathbb{Z}} k_{n}=\sum_{n \in \mathbb{Z}} k_{n}^{\prime}
$$


THE STABILITY OF FULL DIMENSIONAL KAM TORI FOR NONLINEAR SCHRÖDINGER EQUATION

and

$$
\sum_{n \in \mathbb{Z}} n k_{n}=\sum_{n \in \mathbb{Z}} n k_{n}^{\prime}
$$

Define by

$$
\operatorname{supp} \mathcal{M}_{a k k^{\prime}}=\left\{n: 2 a_{n}+k_{n}+k_{n}^{\prime} \neq 0\right\}
$$

and

$$
\text { degree } \mathcal{M}_{a k k^{\prime}}=\sum_{n \in \mathbb{Z}}\left(2 a_{n}+k_{n}+k_{n}^{\prime}\right) \text {. }
$$

With these notations, the Hamiltonian (1.6) has the form of

$$
H(q, \bar{q})=H_{2}(q, \bar{q})+\sum_{a, k, k^{\prime}} B_{a k k^{\prime}} \mathcal{M}_{a k k^{\prime}}
$$

where

$$
H_{2}(q, \bar{q})=\sum_{n \in \mathbb{Z}}\left(n^{2}+V_{n}\right)\left|q_{n}\right|^{2},
$$

$B_{a k k^{\prime}}$ are the coefficients, $V_{n} \in[-1,1]$ for $\forall n \in \mathbb{Z}$ and assuming

$$
\sum_{n \in \mathbb{Z}}\left(2 a_{n}+k_{n}+k_{n}^{\prime}\right)=6
$$

Denote by

$$
n_{1}^{*}=\max \left\{|n|: a_{n}+k_{n}+k_{n}^{\prime} \neq 0\right\} .
$$

Before defining the norm of the Hamiltonian, we give the following lemmas:

Lemma 2.1. Denote $\left(n_{i}^{*}\right)_{i \geq 1}$ the decreasing rearrangement of

$$
\left\{|n|: \text { where } n \text { is repeated } 2 a_{n}+k_{n}+k_{n}^{\prime} \text { times }\right\} \text {, }
$$

and assume

$$
\sum_{n \in \mathbb{Z}}\left(k_{n}-k_{n}^{\prime}\right) n=0 .
$$

Then for any $0<\theta<1$, one has

$$
\sum_{n \in \mathbb{Z}}\left(2 a_{n}+k_{n}+k_{n}^{\prime}\right)|n|^{\theta} \geq 2\left(n_{1}^{*}\right)^{\theta}+\left(2-2^{\theta}\right) \sum_{i \geq 3}\left(n_{i}^{*}\right)^{\theta} .
$$

Proof. Without loss of generality, denote $\left(n_{i}\right),\left|n_{1}\right| \geq\left|n_{2}\right| \geq \cdots$, the system $\{n$ : where $n$ is repeated $2 a_{n}+k_{n}+k_{n}^{\prime}$ times $\}$ and we have $n_{i}^{*}=\left|n_{i}\right|$ for $\forall i \geq 1$. In view of (2.7), there exist $\left(\mu_{i}\right)_{i \geq 1}$ with $\mu_{i} \in\{ \pm 1\}$ such that

$$
\sum_{i \geq 1} \mu_{i} n_{i}=0
$$

and hence

$$
n_{1}^{*} \leq \sum_{i \geq 2}\left|n_{i}\right|
$$

Consequently

$$
\left(n_{1}^{*}\right)^{\theta} \leq\left(\sum_{i \geq 2}\left|n_{i}\right|\right)^{\theta} .
$$


Thus the inequality (2.8) will follow from the inequality

$$
\sum_{i \geq 2}\left|n_{i}\right|^{\theta} \geq\left(\sum_{i \geq 2}\left|n_{i}\right|\right)^{\theta}+\left(2-2^{\theta}\right) \sum_{i \geq 3}\left|n_{i}\right|^{\theta} .
$$

To prove the inequality (2.9), one just needs the following fact: consider the function

$$
f(x)=(1+x)^{\theta}-x^{\theta}, \quad x \in[1, \infty),
$$

and one has

$$
\max _{x \in[1, \infty)} f(x)=f(1)=2^{\theta}-1
$$

which is based on

$$
f^{\prime}(x)=\theta\left((1+x)^{\theta-1}-x^{\theta-1}\right)<0, \text { for } x \in[1, \infty) \text { and } \forall \theta \in(0,1) .
$$

Hence, for any $a \geq b>0$, we have

$$
\begin{aligned}
(a+b)^{\theta}+\left(2-2^{\theta}\right) b^{\theta}-a^{\theta}-b^{\theta} & =(a+b)^{\theta}-a^{\theta}+\left(1-2^{\theta}\right) b^{\theta} \\
& =b^{\theta}\left(\left(1+\frac{a}{b}\right)^{\theta}-\left(\frac{a}{b}\right)^{\theta}-\left(2^{\theta}-1\right)\right) \\
& \leq 0 \text { (in view of (2.10) }),
\end{aligned}
$$

that is

$$
a^{\theta}+b^{\theta} \geq(a+b)^{\theta}+\left(2-2^{\theta}\right) b^{\theta} \quad(\text { for } \forall a \geq b>0) .
$$

By iteration, one obtains

$$
\begin{aligned}
\sum_{i \geq 2}\left|n_{i}\right|^{\theta}= & \left|n_{2}\right|^{\theta}+\left|n_{3}\right|^{\theta}+\sum_{i \geq 4}\left|n_{i}\right|^{\theta} \\
\geq & \left.\left(\left|n_{2}\right|+\left|n_{3}\right|\right)^{\theta}+\left(2-2^{\theta}\right)\left|n_{3}\right|^{\theta}+\sum_{i \geq 4}\left|n_{i}\right|^{\theta} \quad \text { (in view of (2.11) }\right) \\
= & \left(\left|n_{2}\right|+\left|n_{3}\right|\right)^{\theta}+\left|n_{4}\right|^{\theta}+\sum_{i \geq 5}\left|n_{i}\right|^{\theta}+\left(2-2^{\theta}\right)\left|n_{3}\right|^{\theta} \\
\geq & \left(\left|n_{2}\right|+\left|n_{3}\right|+\left|n_{4}\right|\right)^{\theta}+\left(2-2^{\theta}\right)\left|n_{4}\right|^{\theta}+\sum_{i \geq 5}\left|n_{i}\right|^{\theta}+\left(2-2^{\theta}\right)\left|n_{3}\right|^{\theta} \\
= & \left(\left|n_{2}\right|+\left|n_{3}\right|+\left|n_{4}\right|\right)^{\theta}+\sum_{i \geq 5}\left|n_{i}\right|^{\theta}+\left(2-2^{\theta}\right)\left(\left|n_{3}\right|^{\theta}+\left|n_{4}\right|^{\theta}\right) \\
& \cdots \\
\geq & \left(\sum_{i \geq 2}\left|n_{i}\right|\right)^{\theta}+\left(2-2^{\theta}\right)\left(\sum_{i \geq 3}\left|n_{i}\right|^{\theta}\right) .
\end{aligned}
$$

Now we will define the norm of the Hamiltonian $\sum_{a, k, k^{\prime}} B_{a k k^{\prime}} \mathcal{M}_{a k k^{\prime}}$ with the weight $\rho>0$ by

Definition 2.2.

$$
\|H\|_{\rho}=\max _{a, k, k^{\prime}} \frac{\left|B_{a k k^{\prime}}\right|}{e^{\rho \sum_{n}\left(\left(2 a_{n}+k_{n}+k_{n}^{\prime}\right)|n|^{\theta}-2\left(n_{1}^{*}\right)^{\theta}\right)} .}
$$




\section{The Homological Equations}

3.1. Derivation of homological equations. The proof of Main Theorem employs the rapidly converging iteration scheme of Newton type to deal with small divisor problems introduced by Kolmogorov, involving the infinite sequence of coordinate transformations. At the $s$-th step of the scheme, a Hamiltonian $H_{s}=N_{s}+R_{s}$ is considered, as a small perturbation of some normal form $N_{s}$. A transformation $\Phi_{s}$ is set up so that

$$
H_{s} \circ \Phi_{s}=N_{s+1}+R_{s+1}
$$

with another normal form $N_{s+1}$ and a much smaller perturbation $R_{s+1}$. We drop the index $s$ of $H_{s}, N_{s}, R_{s}, \Phi_{s}$ and shorten the index $s+1$ as + .

Now consider the Hamiltonian $H$ of the form

$$
H=N+R,
$$

where

$$
N=\sum_{n \in \mathbb{Z}}\left(n^{2}+\widetilde{V}_{n}\right)\left|q_{n}\right|^{2}
$$

and

$$
R=R_{0}+R_{1}+R_{2}
$$

with $\left|\widetilde{V}_{n}\right| \leq 2$ for all $n \in \mathbb{Z}$,

$$
\begin{aligned}
& R_{0}=\sum_{\substack{a, k, k^{\prime} \\
k \cap \operatorname{supp} \\
k k^{\prime}=\emptyset}} B_{a k k^{\prime}} \mathcal{M}_{a k k^{\prime}}, \\
& R_{1}=\sum_{n \in \mathbb{Z}} J_{n}\left(\sum_{\substack{a, k, k^{\prime} \\
k \cap \operatorname{supp} k^{\prime}=\emptyset}} B_{a k k^{\prime}}^{(n)} \mathcal{M}_{a k k^{\prime}}\right), \\
& R_{2}=\sum_{n_{1}, n_{2} \in \mathbb{Z}} J_{n_{1}} J_{n_{2}}\left(\sum_{\substack{a, k, k^{\prime} \\
\operatorname{supp} \text { assumption }}} B_{a k k^{\prime}}^{\left(n_{1}, n_{2}\right)} \mathcal{M}_{a k k^{\prime}}\right),
\end{aligned}
$$

and

$$
J_{n}=I_{n}-I_{n}(0), \quad I_{n}=\left|q_{n}\right|^{2} .
$$

We desire to eliminate the terms $R_{0}, R_{1}$ in (3.1) by the coordinate transformation $\Phi$, which is obtained as the time-1 map $\left.X_{F}^{t}\right|_{t=1}$ of a Hamiltonian vector field $X_{F}$ with $F=F_{0}+F_{1}$. Let $F_{0}\left(\operatorname{resp} . F_{1}\right)$ has the form of $R_{0}\left(\operatorname{resp} \cdot R_{1}\right)$, that is

$$
\begin{aligned}
& F_{0}=\sum_{\substack{a, k, k^{\prime} \\
\operatorname{supp} \operatorname{Supp}_{k^{\prime}=\emptyset}}} F_{a k k^{\prime}} \mathcal{M}_{a k k^{\prime}}, \\
& F_{1}=\sum_{n \in \mathbb{Z}} J_{n}\left(\sum_{\substack{a, k, k^{\prime} \\
k \cap \operatorname{Supp}_{k^{\prime}=\emptyset}}} F_{a k k^{\prime}}^{(n)} \mathcal{M}_{a k k^{\prime}}\right),
\end{aligned}
$$

and the homological equations become

$$
\{N, F\}+R_{0}+R_{1}=\left[R_{0}\right]+\left[R_{1}\right],
$$


where

$$
\left[R_{0}\right]=\sum_{a} B_{a 00} \mathcal{M}_{a 00}
$$

and

$$
\left[R_{1}\right]=\sum_{n \in \mathbb{Z}} J_{n} \sum_{a} B_{a 00}^{(n)} \mathcal{M}_{a 00} .
$$

The solutions of the homological equations (3.4) are given by

$$
F_{a k k^{\prime}}=\frac{B_{a k k^{\prime}}}{\sum_{n \in \mathbb{Z}}\left(k_{n}-k_{n}^{\prime}\right)\left(n^{2}+\widetilde{V}_{n}\right)},
$$

where

$$
F_{a k k^{\prime}}^{(m)}=\frac{B_{a k k^{\prime}}^{(m)}}{\sum_{n \in \mathbb{Z}}\left(k_{n}-k_{n}^{\prime}\right)\left(n^{2}+\widetilde{V}_{n}\right)},
$$

and the new Hamiltonian $H_{+}$has the form

$$
\begin{aligned}
H_{+}= & H \circ \Phi \\
= & N+\{N, F\}+R_{0}+R_{1} \\
& +\int_{0}^{1}\left\{(1-t)\{N, F\}+R_{0}+R_{1}, F\right\} \circ X_{F}^{t} \mathrm{~d} t+R_{2} \circ X_{F}^{1} \\
= & N_{+}+R_{+},
\end{aligned}
$$

where

$$
N_{+}=N+\left[R_{0}\right]+\left[R_{1}\right]
$$

and

$$
R_{+}=\int_{0}^{1}\left\{(1-t)\{N, F\}+R_{0}+R_{1}, F\right\} \circ X_{F}^{t} \mathrm{~d} t+R_{2} \circ X_{F}^{1} .
$$

3.2. The solutions of the homological equations. In this subsection, we will estimate the solutions of the homological equations. To this end, we define a new norm for the Hamiltonian $R$ of the form as in (3.1) as follows:

$$
\|R\|_{\rho}^{+}=\max \left\{\left\|R_{0}\right\|_{\rho}^{+},\left\|R_{1}\right\|_{\rho}^{+} \mid,\left\|R_{2}\right\|_{\rho}^{+}\right\},
$$

where

$$
\begin{aligned}
& \left\|R_{0}\right\|_{\rho}^{+}=\sup _{a, k, k^{\prime}} \frac{\left|B_{a k k^{\prime}}\right|}{e^{\rho\left(\sum_{n}\left(2 a_{n}+k_{n}+k_{n}^{\prime}\right)|n|^{\theta}-2\left(n_{1}^{*}\right)^{\theta}\right)}}, \\
& \left\|R_{1}\right\|_{\rho}^{+}=\sup _{\substack{a, k, k^{\prime} \\
m \in \mathbb{Z}}} \frac{\left|B_{a k k^{\prime}}^{(m)}\right|}{e^{\rho\left(\sum_{n}\left(2 a_{n}+k_{n}+k_{n}^{\prime}\right)|n|^{\theta}+2|m|^{\theta}-2\left(n_{1}^{*}\right)^{\theta}\right)}}, \\
& \left\|R_{2}\right\|_{\rho}^{+}=\sup _{\substack{a, k, k^{\prime} \\
m_{1}, m_{2} \in \mathbb{Z}}} \frac{\left|B_{a k k^{\prime}}^{\left(m_{1}, m_{2}\right)}\right|}{e^{\rho\left(\sum_{n}\left(2 a_{n}+k_{n}+k_{n}^{\prime}\right)|n|^{\theta}+2\left|m_{1}\right|^{\theta}+2\left|m_{2}\right|^{\theta}-2\left(n_{1}^{*}\right)^{\theta}\right)}} .
\end{aligned}
$$

Moreover, one has the following estimates: 
THE STABILITY OF FULL DIMENSIONAL KAM TORI FOR NONLINEAR SCHRÖDINGER EQUATIOIg

Lemma 3.1. Given any $\rho, \delta>0$ and a Hamiltonian $R$, one has

$$
\|R\|_{\rho+\delta}^{+} \leq\left(\frac{1}{\delta}\right)^{C(\theta) \delta^{-\frac{1}{\theta}}}\|R\|_{\rho}
$$

and

$$
\|R\|_{\rho+\delta} \leq \frac{C(\theta)}{\delta^{2}}\|R\|_{\rho}^{+}
$$

where $C(\theta)$ is a positive constant depending on $\theta$ only.

Proof. See the details of the proof in the Appendix.

Lemma 3.2. Given $\theta \in(0,1)$, let $\left(\widetilde{V}_{n}\right)$ be Diophantine with $\gamma>0$ (see (1.8)). Then for any $\rho>0,0<\delta \ll 1$ (depending only on $\theta$ ), the solutions of the homological equations (3.4), which are given by (3.7) and (3.8), satisfy

$$
\left\|F_{i}\right\|_{\rho+\delta}^{+} \leq \frac{1}{\gamma} \cdot e^{C(\theta) \delta^{-\frac{5}{\theta}}}\left\|R_{i}\right\|_{\rho}^{+},
$$

where $i=0,1$ and $C(\theta)$ is a positive constant depending on $\theta$ only.

Proof. We distinguish two cases:

Case. 1.

$$
\left|\sum_{n \in \mathbb{Z}}\left(k_{n}-k_{n}^{\prime}\right) n^{2}\right|>10 \sum_{n \in \mathbb{Z}}\left|k_{n}-k_{n}^{\prime}\right| .
$$

Since $\left|\widetilde{V}_{n}\right| \leq 2$, we have

$$
\left|\sum_{n \in \mathbb{Z}}\left(k_{n}-k_{n}^{\prime}\right)\left(n^{2}+\widetilde{V}_{n}\right)\right|>10 \sum_{n \in \mathbb{Z}}\left|k_{n}-k_{n}^{\prime}\right|-2 \sum_{n \in \mathbb{Z}}\left|k_{n}-k_{n}^{\prime}\right| \geq 1,
$$

where the last inequality is based on $\operatorname{supp} k \bigcap \operatorname{supp} k^{\prime}=\emptyset$. There is no small divisor and (3.18) holds trivially.

Case. 2.

$$
\left|\sum_{n \in \mathbb{Z}}\left(k_{n}-k_{n}^{\prime}\right) n^{2}\right| \leq 10 \sum_{n \in \mathbb{Z}}\left|k_{n}-k_{n}^{\prime}\right| .
$$

In this case, we always assume

$$
\left|\sum_{n \in \mathbb{Z}}\left(k_{n}-k_{n}^{\prime}\right)\left(n^{2}+\widetilde{V}_{n}\right)\right| \leq 1,
$$

otherwise there is no small divisor.

Firstly, one has

$$
\begin{aligned}
\sum_{n \in \mathbb{Z}}\left|k_{n}-k_{n}^{\prime}\right||n|^{\theta / 2} & \leq 3 \cdot 6^{\theta / 2}\left(\sum_{i \geq 3}\left(n_{i}^{*}\right)^{\theta}\right) \quad \text { (in view of Lemma 7.1) } \\
& \leq \frac{3 \cdot 6^{\theta / 2}}{2-2^{\theta}}\left(\sum_{n \in \mathbb{Z}}\left(2 a_{n}+k_{n}+k_{n}^{\prime}\right)|n|^{\theta}-2\left(n_{1}^{*}\right)^{\theta}\right),
\end{aligned}
$$

where the last inequality is based on Lemma 2.1.

Since

$$
\sum_{n \in \mathbb{Z}}\left(k_{n}-k_{n}^{\prime}\right) n^{2} \in \mathbb{Z},
$$


the Diophantine property of $\left(\widetilde{V}_{n}\right)$ implies

$$
\left|\sum_{n \in \mathbb{Z}}\left(k_{n}-k_{n}^{\prime}\right)\left(n^{2}+\widetilde{V}_{n}\right)\right| \geq \frac{\gamma}{2} \prod_{n \in \mathbb{Z}} \frac{1}{1+\left|k_{n}-k_{n}^{\prime}\right|^{2}|n|^{4}} .
$$

Hence,

$$
\begin{aligned}
& \left|F_{a k k^{\prime}}\right| e^{-(\rho+\delta)\left(\sum_{n}\left(2 a_{n}+k_{n}+k_{n}^{\prime}\right)|n|^{\theta}-2\left(n_{1}^{*}\right)^{\theta}\right)} \\
= & \frac{\left|B_{a k k^{\prime}}\right|}{\left|\sum_{n}\left(k_{n}-k_{n}^{\prime}\right)\left(n^{2}+\widetilde{V}_{n}\right)\right|} e^{-(\rho+\delta)\left(\sum_{n}\left(2 a_{n}+k_{n}+k_{n}^{\prime}\right)|n|^{\theta}-2\left(n_{1}^{*}\right)^{\theta}\right)} \\
= & \quad \text { in view of (B.7)) } \\
& \left|B_{a k k^{\prime}}\right| e^{-\rho\left(\sum_{n}\left(2 a_{n}+k_{n}+k_{n}^{\prime}\right)|n|^{\theta}-2\left(n_{1}^{*}\right)^{\theta}\right)} \\
& \times \frac{e^{-\delta\left(\sum_{n}\left(2 a_{n}+k_{n}+k_{n}^{\prime}\right)|n|^{\theta}-2\left(n_{1}^{*}\right)^{\theta}\right)}}{\left|\sum_{n}\left(k_{n}-k_{n}^{\prime}\right)\left(n^{2}+\widetilde{V}_{n}\right)\right|} \\
\leq & \left.2 \gamma^{-1}|| R_{0}\right|_{\rho} ^{+} \prod_{n}\left(1+\left|k_{n}-k_{n}^{\prime}\right|^{2}|n|^{4}\right) e^{-\delta\left(\sum_{n}\left(2 a_{n}+k_{n}+k_{n}^{\prime}\right)|n|^{\theta}-2\left(n_{1}^{*}\right)^{\theta}\right)}
\end{aligned}
$$

(in view of (3.13) and (3.20)

$$
\leq 2 \gamma^{-1}|| R_{0} \|_{\rho}^{+} e^{\sum_{n} \ln \left(1+\left|k_{n}-k_{n}^{\prime}\right|^{2}|n|^{4}\right)} e^{-\frac{3 \cdot 6^{\theta / 2} \delta}{2-2^{\theta}} \sum_{n}\left(\left|k_{n}-k_{n}^{\prime}\right||n|^{\theta / 2}\right)}
$$

(in view of (3.19) )

$$
\leq 2 \gamma^{-1}|| R_{0} \|_{\rho}^{+} e^{\sum_{n} \ln \left(1+\left|k_{n}-k_{n}^{\prime}\right|^{2}|n|^{4}\right)} e^{-\delta \sum_{n}\left(\left|k_{n}-k_{n}^{\prime}\right||n|^{\theta / 2}\right)}
$$

(in view of $\frac{3 \cdot 6^{\theta / 2}}{2-2^{\theta}}>1$ )

$$
\begin{aligned}
& =2 \gamma^{-1}\left\|R_{0}\right\|_{\rho}^{+} e^{\sum_{n: k_{n} \neq k_{n}^{\prime}} \ln \left(1+\left|k_{n}-k_{n}^{\prime}\right|^{2}|n|^{4}\right)-\delta \sum_{n: k_{n} \neq k_{n}^{\prime}}\left(\left|k_{n}-k_{n}^{\prime}\right||n|^{\theta / 2}\right)} \\
& \leq 2 \gamma^{-1}\left\|R_{0}\right\|_{\rho}^{+} e^{8\left(\sum_{n: k_{n} \neq k_{n}^{\prime}} \ln \left(\left|k_{n}-k_{n}^{\prime}\right||n|\right)\right)+3-\delta \sum_{n: k_{n} \neq k_{n}^{\prime}}\left(\left|k_{n}-k_{n}^{\prime}\right|^{\theta / 2}|n|^{\theta / 2}\right)}
\end{aligned}
$$

(in view of $0<\theta<1$ )

$$
\begin{aligned}
& =\frac{2 e^{3}}{\gamma}\left\|R_{0}\right\|_{\rho}^{+} e^{\sum_{n: k_{n} \neq k_{n}^{\prime}}\left(8 \ln \left(\left|k_{n}-k_{n}^{\prime}\right||n|\right)-\delta\left|k_{n}-k_{n}^{\prime}\right|^{\theta / 2}|n|^{\theta / 2}\right)} \\
& =\frac{2 e^{3}}{\gamma}\left\|R_{0}\right\|_{\rho}^{+} e^{\sum_{|n| \leq N: k_{n} \neq k_{n}^{\prime}}\left(8 \ln \left(\left|k_{n}-k_{n}^{\prime}\right||n|\right)-\delta\left|k_{n}-k_{n}^{\prime}\right|^{\theta / 2}|n|^{\theta / 2}\right)}
\end{aligned}
$$$$
+\frac{2 e^{3}}{\gamma}\left\|R_{0}\right\|_{\rho}^{+} e^{\sum_{n>N: k_{n} \neq k_{n}^{\prime}}\left(8 \ln \left(\left|k_{n}-k_{n}^{\prime}\right||n|\right)-\delta\left|k_{n}-k_{n}^{\prime}\right|^{\theta / 2}|n|^{\theta / 2}\right)}
$$

(where $N=\left(\frac{16}{\theta \delta}\right)^{4 / \theta}$ )

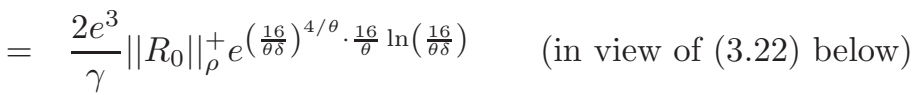

$$
\begin{aligned}
& +\frac{2 e^{3}}{\gamma}\left\|R_{0}\right\|_{\rho}^{+} \quad \text { (in view of (13.23) below) }
\end{aligned}
$$

$$
\leq \frac{1}{\gamma} \cdot e^{C(\theta) \delta^{-\frac{5}{\theta}}}\left\|R_{0}\right\|_{\rho}^{+} \quad(\text { for } 0<\delta \ll 1)
$$

where $C(\theta)$ is a positive constant depending on $\theta$ only.

Therefore, in view of (3.13) and (3.21), we finish the proof of (3.18) for $i=0$. 
THE STABILITY OF FULL DIMENSIONAL KAM TORI FOR NONLINEAR SCHRÖDINGER EQUATIOIN

It is easy to verify the following two facts that

$$
\max _{x \geq 1} f(x)=f\left(\left(\frac{16}{\theta \delta}\right)^{2 / \theta}\right)=-\frac{16}{\theta}+8 \ln \left(\left(\frac{16}{\theta \delta}\right)^{2 / \theta}\right) \leq \frac{16}{\theta} \ln \left(\frac{16}{\theta \delta}\right)
$$

with $f(x)=\left(-\delta x^{\theta / 2}+8 \ln x\right)$, and when $|n|>N=\left(\frac{16}{\theta \delta}\right)^{4 / \theta}, k_{n} \neq k_{n}^{\prime}$, one has

$$
-\delta\left(\left|k_{n}-k_{n}^{\prime}\right|^{\theta / 2}|n|^{\theta / 2}\right)+8 \ln \left(\left|k_{n}-k_{n}^{\prime}\right||n|\right)<0 \quad(\text { for } 0<\delta \ll 1) .
$$

Similarly, one can prove (3.18) for $i=1$.

\section{The NeW Hamiltonian}

In view of (3.9), we obtain the new Hamiltonian

$$
H_{+}=H \circ \Phi=N_{+}+R_{+},
$$

where $N_{+}$and $R_{+}$are given in (3.10) and 3.11) respectively.

4.1. Estimating Poisson Bracket and Symplectic Transformation. To estimate the Hamiltonian $H_{+}$, we need the following two lemmas.

Lemma 4.1. Let $\theta \in(0,1), \rho>0$ and $0<\delta_{1}, \delta_{2} \ll 1$ (depending on $\theta, \rho$ ). Then one has

$$
\left\|\left\{H_{1}, H_{2}\right\}\right\|_{\rho} \leq \frac{1}{\delta_{2}}\left(\frac{1}{\delta_{1}}\right)^{C(\theta) \delta_{1}^{-\frac{1}{\theta}}}\left\|H_{1}\right\|_{\rho-\delta_{1}}\left\|H_{2}\right\|_{\rho-\delta_{2}},
$$

where $C(\theta)$ is a positive constant depending on $\theta$ only.

Proof. Let

$$
H_{1}=\sum_{a, k, k^{\prime}} b_{a k k^{\prime}} \mathcal{M}_{a k k^{\prime}}
$$

and

$$
H_{2}=\sum_{A, K, K^{\prime}} B_{A K K^{\prime}} \mathcal{M}_{A K K^{\prime}}
$$

Hence

$$
\left\{H_{1}, H_{2}\right\}=\sum_{a, k, k^{\prime}, A, K, K^{\prime}} b_{a k k^{\prime}} B_{A K K^{\prime}}\left\{\mathcal{M}_{a k k^{\prime}}, \mathcal{M}_{A K K^{\prime}}\right\} .
$$

Write

$$
\begin{aligned}
\left\{\mathcal{M}_{a k k^{\prime}}, \mathcal{M}_{A K K^{\prime}}\right\}= & \frac{1}{2 \mathbf{i}} \sum_{j}\left(\prod_{n \neq j} I_{n}(0)^{a_{n}+A_{n}} q_{n}^{k_{n}+K_{n}} \bar{q}_{n}^{k_{n}^{\prime}+K_{n}^{\prime}}\right) \\
& \times\left(\left(k_{j} K_{j}^{\prime}-k_{j}^{\prime} K_{j}\right) I_{j}(0)^{a_{j}+A_{j}} q_{j}^{k_{j}+K_{j}-1} \bar{q}_{j}^{k_{j}^{\prime}+K_{j}^{\prime}-1}\right) .
\end{aligned}
$$

Then the coefficient of $\prod_{n} I_{n}(0)^{\alpha_{n}} q_{n}^{\kappa_{n}} \bar{q}_{n}^{\kappa_{n}^{\prime}}=\mathcal{M}_{\alpha \kappa \kappa^{\prime}}$ is given by

$$
2 \mathbf{i} B_{\alpha \kappa \kappa^{\prime}}=\sum_{j} \sum_{*} \sum_{* *}\left(k_{j} K_{j}^{\prime}-k_{j}^{\prime} K_{j}\right) b_{a k k^{\prime}} B_{A K K^{\prime}},
$$

where

$$
\sum_{*}=\sum_{\substack{a, A \\ a+A=\alpha}}
$$


and

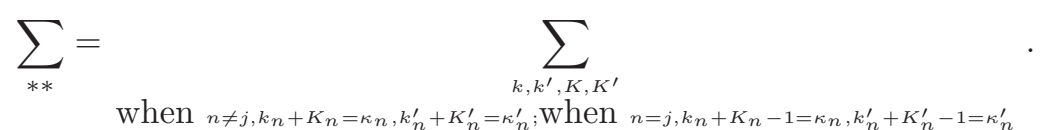

Remark 4.2. Note that

$$
\sum_{n}\left(2 \alpha_{n}+\kappa_{n}+\kappa_{n}^{\prime}\right)=\sum_{n}\left(2 a_{n}+k_{n}+k_{n}^{\prime}\right)+\sum_{n}\left(2 A_{n}+K_{n}+K_{n}^{\prime}\right)-2
$$

and

$$
\sum_{n}\left(2 \alpha_{n}+\kappa_{n}+\kappa_{n}^{\prime}\right)|n|=\sum_{n}\left(2 a_{n}+k_{n}+k_{n}^{\prime}\right)|n|+\sum_{n}\left(2 A_{n}+K_{n}+K_{n}^{\prime}\right)|n|-2|j| .
$$

In view of (2.12), one has

$$
\begin{aligned}
\left|b_{a k k^{\prime}}\right| & \leq\left\|H_{1}\right\|_{\rho-\delta_{1}} e^{\left(\rho-\delta_{1}\right) \sum_{n}\left(2 a_{n}+k_{n}+k_{n}^{\prime}\right)|n|^{\theta}-2\left(\rho-\delta_{1}\right)\left(n_{1}^{*}\right)^{\theta}} \\
& =\left\|H_{1}\right\|_{\rho-\delta_{1}} e^{\rho \sum_{n}\left(2 a_{n}+k_{n}+k_{n}^{\prime}\right)|n|^{\theta}-2 \rho\left(n_{1}^{*}\right)^{\theta}} e^{-\delta_{1} \sum_{n}\left(2 a_{n}+k_{n}+k_{n}^{\prime}\right)|n|^{\theta}+2 \delta_{1}\left(n_{1}^{*}\right)^{\theta}} \\
(4.6) & \leq\left\|H_{1}\right\|_{\rho-\delta_{1}} e^{\rho \sum_{n}\left(2 a_{n}+k_{n}+k_{n}^{\prime}\right)|n|^{\theta}-2 \rho\left(n_{1}^{*}\right)^{\theta}} e^{-\left(2-2^{\theta}\right) \delta_{1} \sum_{i \geq 3}\left(n_{i}^{*}\right)^{\theta}}
\end{aligned}
$$

where the last inequality is based on Lemma 2.1 .

Similarly,

$$
\left|B_{A K K^{\prime}}\right| \leq \|\left. H_{2}\right|_{\rho-\delta_{2}} e^{\rho \sum_{n}\left(2 A_{n}+K_{n}+K_{n}^{\prime}\right)|n|^{\theta}-2 \rho\left(N_{1}^{*}\right)^{\theta}} e^{-\left(2-2^{\theta}\right) \delta_{2} \sum_{i \geq 3}\left(N_{i}^{*}\right)^{\theta}} .
$$

Substitution of (4.6) and (4.7) in (4.3) gives

$$
\begin{aligned}
\left|2 \mathbf{i} B_{\alpha \kappa \kappa^{\prime}}\right| \leq & || H_{1}\left\|_{\rho-\delta_{1}}|| H_{2}\right\|_{\rho-\delta_{2}} \sum_{j} \sum_{*} \sum_{* *}\left|k_{j} K_{j}^{\prime}-k_{j}^{\prime} K_{j}\right| \\
& \times e^{\rho\left(\sum_{n}\left(2 a_{n}+k_{n}+k_{n}^{\prime}\right)|n|^{\theta}-2\left(n_{1}^{*}\right)^{\theta}+\sum_{n}\left(2 A_{n}+K_{n}+K_{n}^{\prime}\right)|n|^{\theta}-2\left(N_{1}^{*}\right)^{\theta}\right)} \\
& \times e^{-\left(2-2^{\theta}\right) \delta_{1} \sum_{i \geq 3}\left(n_{i}^{*}\right)^{\theta}} e^{-\left(2-2^{\theta}\right) \delta_{2} \sum_{i \geq 3}\left(N_{i}^{*}\right)^{\theta}} \\
= & \left\|H_{1}\right\|_{\rho-\delta_{1}}|| H_{2} \|_{\rho-\delta_{2}} \sum_{j} \sum_{*} \sum_{* *}\left|k_{j} K_{j}^{\prime}-k_{j}^{\prime} K_{j}\right| \\
& \times e^{\rho\left(\sum_{n}\left(2 \alpha_{n}+\kappa_{n}+\kappa_{n}^{\prime}\right)|n|^{\theta}+2|j|^{\theta}\right)} e^{-2 \rho\left(n_{1}^{*}\right)^{\theta}-2 \rho\left(N_{1}^{*}\right)^{\theta}} \\
& \times e^{-\left(2-2^{\theta}\right) \delta_{1} \sum_{i \geq 3}\left(n_{i}^{*}\right)^{\theta}} e^{-\left(2-2^{\theta}\right) \delta_{2} \sum_{i \geq 3}\left(N_{i}^{*}\right)^{\theta}}
\end{aligned}
$$

(in view of 4.51)

$$
\begin{aligned}
= & \left\|H_{1}\right\|_{\rho-\delta_{1}}|| H_{2}||_{\rho-\delta_{2}} e^{\rho\left(\sum_{n}\left(2 \alpha_{n}+\kappa_{n}+\kappa_{n}^{\prime}\right)|n|^{\theta}-2\left(\nu_{1}^{*}\right)^{\theta}\right)} \\
& \times \sum_{j} \sum_{*} \sum_{* *}\left|k_{j} K_{j}^{\prime}-k_{j}^{\prime} K_{j}\right| e^{2 \rho\left(|j|^{\theta}+\left(\nu_{1}^{*}\right)^{\theta}-\left(n_{1}^{*}\right)^{\theta}-\left(N_{1}^{*}\right)^{\theta}\right)} \\
& \times e^{-\left(2-2^{\theta}\right) \delta_{1} \sum_{i \geq 3}\left(n_{i}^{*}\right)^{\theta}} e^{-\left(2-2^{\theta}\right) \delta_{2} \sum_{i \geq 3}\left(N_{i}^{*}\right)^{\theta}},
\end{aligned}
$$

where

$$
\nu_{1}^{*}=\max \left\{|n|: \alpha_{n}+\kappa_{n}+\kappa_{n}^{\prime} \neq 0\right\} .
$$

To show (4.2) holds, it suffices to prove

$$
I \leq \frac{1}{\delta_{2}}\left(\frac{1}{\delta_{1}}\right)^{C(\theta) \delta_{1}^{-\frac{1}{\theta}}}
$$


THE STABILITY OF FULL DIMENSIONAL KAM TORI FOR NONLINEAR SCHRÖDINGER EQUATIOI

where

$$
\begin{aligned}
I= & \sum_{j} \sum_{*} \sum_{* *}\left|k_{j} K_{j}^{\prime}-k_{j}^{\prime} K_{j}\right| e^{2 \rho\left(|j|^{\theta}+\left(\nu_{1}^{*}\right)^{\theta}-\left(n_{1}^{*}\right)^{\theta}-\left(N_{1}^{*}\right)^{\theta}\right)} \\
& \times e^{-\left(2-2^{\theta}\right) \delta_{1} \sum_{i \geq 3}\left|n_{i}\right|^{\theta}} e^{-\left(2-2^{\theta}\right) \delta_{2} \sum_{i \geq 3}\left|N_{i}\right|^{\theta}} .
\end{aligned}
$$

To this end, we first note some simple facts:

1. If $j \notin \operatorname{supp}\left(k+k^{\prime}\right) \bigcup \operatorname{supp}\left(K+K^{\prime}\right)$, then

$$
\frac{\partial \mathcal{M}_{a k k^{\prime}}}{\partial q_{j}} \frac{\partial \mathcal{M}_{A K K^{\prime}}}{\partial \bar{q}_{j}}-\frac{\partial \mathcal{M}_{a k k^{\prime}}}{\partial \bar{q}_{j}} \frac{\partial \mathcal{M}_{A K K^{\prime}}}{\partial q_{j}}=0 .
$$

Hence we always assume $j \in \operatorname{supp}\left(k+k^{\prime}\right) \bigcup \operatorname{supp}\left(K+K^{\prime}\right)$. Therefore one has

$$
|j| \leq \min \left\{n_{1}^{*}, N_{1}^{*}\right\}
$$

2. The following inequality always holds

$$
\nu_{1}^{*} \leq \max \left\{n_{1}^{*}, N_{1}^{*}\right\},
$$

and then one has

$$
|j|^{\theta}+\left(\nu_{1}^{*}\right)^{\theta}-\left(n_{1}^{*}\right)^{\theta}-\left(N_{1}^{*}\right)^{\theta} \leq 0
$$

3. It is easy to see

$$
\begin{aligned}
\sum_{i \geq 1}\left(n_{i}^{*}\right)^{\theta} & =\sum_{n}\left(2 a_{n}+k_{n}+k_{n}^{\prime}\right)|n|^{\theta} \\
& \geq \sum_{n}\left(2 a_{n}+k_{n}+k_{n}^{\prime}\right) \\
& \geq \sum_{n}\left(k_{n}+k_{n}^{\prime}\right)
\end{aligned}
$$

and

$$
\begin{aligned}
\sum_{i \geq 3}\left(N_{i}^{*}\right)^{\theta} & \geq \sum_{n}\left(2 A_{n}+K_{n}+K_{n}^{\prime}\right)-2 \\
& \geq \frac{1}{2} \sum_{n}\left(2 A_{n}+K_{n}+K_{n}^{\prime}\right) \\
& \geq \frac{1}{2} \sum_{n}\left(K_{n}+K_{n}^{\prime}\right) .
\end{aligned}
$$

Based on (4.10) and (4.11), we obtain

$$
\begin{aligned}
\sum_{n}\left(k_{n}+k_{n}^{\prime}\right)\left(K_{n}+K_{n}^{\prime}\right) & \leq\left(\sup _{n}\left(K_{n}+K_{n}^{\prime}\right)\right)\left(\sum_{n}\left(k_{n}+k_{n}^{\prime}\right)\right) \\
& \leq 2\left(\sum_{i \geq 1}\left(n_{i}^{*}\right)^{\theta}\right)\left(\sum_{i \geq 3}\left(N_{i}^{*}\right)^{\theta}\right) .
\end{aligned}
$$

Now we will prove the inequality (4.8) holds:

Case. 1. $\nu_{1}^{*} \leq N_{1}^{*}$.

Case. 1.1. $|\bar{j}| \leq n_{3}^{*}$.

Then one has

$$
\begin{aligned}
e^{2 \rho\left(|j|^{\theta}-\left(n_{1}^{*}\right)^{\theta}\right)} & \leq e^{2 \rho\left(\left(n_{3}^{*}\right)^{\theta}-\left(n_{1}^{*}\right)^{\theta}\right)} \\
& \leq e^{\left(2-2^{\theta}\right) \delta_{1}\left(\left(n_{3}^{*}\right)^{\theta}-\left(n_{1}^{*}\right)^{\theta}\right)}
\end{aligned}
$$


where using $0<\delta_{1} \ll 1$ depending on $\theta$ and $\rho$. Hence

$$
\begin{aligned}
& e^{2 \rho\left(|j|^{\theta}+\left(\nu_{1}^{*}\right)^{\theta}-\left(n_{1}^{*}\right)^{\theta}-\left(N_{1}^{*}\right)^{\theta}\right)} e^{-\left(2-2^{\theta}\right) \delta_{1} \sum_{i \geq 3}\left(n_{i}^{*}\right)^{\theta}} \\
\leq & e^{\left(2-2^{\theta}\right) \delta_{1}\left(\left(n_{3}^{*}\right)^{\theta}-\left(n_{1}^{*}\right)^{\theta}\right)} e^{-\left(2-2^{\theta}\right) \delta_{1} \sum_{i \geq 3}\left(n_{i}^{*}\right)^{\theta}} \\
& \left(\text { in view of } \nu_{1}^{*} \leq N_{1}^{*} \text { and (4.13) }\right) \\
= & e^{-\left(2-2^{\theta}\right) \delta_{1}\left(\left(n_{1}^{*}\right)^{\theta}+\sum_{i \geq 4}\left(n_{i}^{*}\right)^{\theta}\right)} \\
\leq & e^{-\frac{\left(2-2^{\theta}\right) \delta_{1}}{3} \sum_{i \geq 1}\left(n_{i}^{*}\right)^{\theta}} .
\end{aligned}
$$

Remark 4.3. Note that if $j, a, k, k^{\prime}$ are specified, and then $A, K, K^{\prime}$ are uniquely determined.

In view of (4.14), we have

$$
\begin{aligned}
& I \leq \sum_{j} \sum_{*} \sum_{* *}\left(k_{j}+k_{j}^{\prime}\right)\left(K_{j}+K_{j}^{\prime}\right) e^{-\frac{\left(2-2^{\theta}\right) \delta_{1}}{3} \sum_{i \geq 1}\left(n_{i}^{*}\right)^{\theta}} e^{-\left(2-2^{\theta}\right) \delta_{2} \sum_{i \geq 3}\left(N_{i}^{*}\right)^{\theta}} \\
& =\sum_{a, k, k^{\prime}} \sum_{j}\left(k_{j}+k_{j}^{\prime}\right)\left(K_{j}+K_{j}^{\prime}\right) e^{-\frac{\left(2-2^{\theta}\right) \delta_{1}}{3} \sum_{i \geq 1}\left(n_{i}^{*}\right)^{\theta}} e^{-\left(2-2^{\theta}\right) \delta_{2} \sum_{i \geq 3}\left(N_{i}^{*}\right)^{\theta}} \\
& \text { (in view of Remark 4.3, one has } \sum_{a, k, k^{\prime}} \sum_{j}=\sum_{*} \sum_{* *} \sum_{j} \text { ) } \\
& \leq \sum_{a, k, k^{\prime}} 2\left(\sum_{i \geq 1}\left(n_{i}^{*}\right)^{\theta}\right)\left(\sum_{i \geq 3}\left(N_{i}^{*}\right)^{\theta}\right) e^{-\frac{\left(2-2^{\theta}\right) \delta_{1}}{3} \sum_{i \geq 1}\left(n_{i}^{*}\right)^{\theta}} e^{-\left(2-2^{\theta}\right) \delta_{2} \sum_{i \geq 3}\left(N_{i}^{*}\right)^{\theta}} \\
& \text { (in view of the inequality (4.15)) } \\
& =2 \sum_{a, k, k^{\prime}}\left(\sum_{i \geq 1}\left(n_{i}^{*}\right)^{\theta} e^{-\frac{\left(2-2^{\theta}\right) \delta_{1}}{3} \sum_{i \geq 1}\left(n_{i}^{*}\right)^{\theta}}\right)\left(\sum_{i \geq 3}\left(N_{i}^{*}\right)^{\theta} e^{-\left(2-2^{\theta}\right) \delta_{2} \sum_{i \geq 3}\left|N_{i}\right|^{\theta}}\right) \\
& \leq \frac{2}{\left(2-2^{\theta}\right) \delta_{2}} \sum_{a, k, k^{\prime}} \sum_{i \geq 1}\left(n_{i}^{*}\right)^{\theta} e^{-\frac{\left(2-2^{\theta}\right) \delta_{1}}{3} \sum_{i \geq 1}\left(n_{i}^{*}\right)^{\theta}} \quad \text { (in view of }(\mathbf{7 . 2 4}) \text { ) } \\
& \leq \frac{24}{\left(2-2^{\theta}\right)^{2} \delta_{1} \delta_{2}} \sum_{a, k, k^{\prime}} e^{-\frac{\left(2-2^{\theta}\right) \delta_{1}}{4} \sum_{i \geq 1}\left(n_{i}^{*}\right)^{\theta}} \quad \text { (in view of (7.24) again) } \\
& =\frac{24}{\left(2-2^{\theta}\right)^{2} \delta_{1} \delta_{2}} \sum_{a, k, k^{\prime}} e^{-\frac{\left(2-2^{\theta}\right) \delta_{1}}{4} \sum_{n}\left(2 a_{n}+k_{n}+k_{n}^{\prime}\right)|n|^{\theta}} \\
& \leq \frac{24}{\left(2-2^{\theta}\right)^{2} \delta_{1} \delta_{2}}\left(\sum_{a} e^{-\frac{\left(2-2^{\theta}\right) \delta_{1}}{4} \sum_{n} 2 a_{n}|n|^{\theta}}\right)\left(\sum_{k} e^{-\frac{\left(2-2^{\theta}\right) \delta_{1}}{4} \sum_{n} k_{n}|n|^{\theta}}\right)^{2} \\
& \leq \frac{24}{\left(2-2^{\theta}\right)^{2} \delta_{1} \delta_{2}} \prod_{n \in \mathbb{Z}}\left(1-e^{-\frac{\left(2-2^{\theta}\right) \delta_{1}}{2}|n|^{\theta}}\right)^{-1}\left(1-e^{-\frac{\left(2-2^{\theta}\right) \delta_{1}}{4}|n|^{\theta}}\right)^{-2}
\end{aligned}
$$

(which is based on Lemma 7.2)

$$
\begin{aligned}
& \left.\leq \frac{C_{1}(\theta)}{\delta_{1} \delta_{2}}\left(\frac{1}{\delta_{1}}\right)^{C_{2}(\theta) \delta_{1}^{-\frac{1}{\theta}}} \quad \text { (in view of (17.16) }\right) \\
& \leq \frac{1}{\delta_{2}}\left(\frac{1}{\delta_{1}}\right)^{C(\theta) \delta_{1}^{-\frac{1}{\theta}}},
\end{aligned}
$$


THE STABILITY OF FULL DIMENSIONAL KAM TORI FOR NONLINEAR SCHRÖDINGER EQUATIOIJ

where the last inequality is based on $0<\delta_{1} \ll 1$ and $C(\theta), C_{1}(\theta), C_{2}(\theta)$ are positive constants depending on $\theta$ only.

Case. 1.2. $j \in\left\{n_{1}, n_{2}\right\},\left|n_{1}\right|=n_{1}^{*},\left|n_{2}\right|=n_{2}^{*}$.

If $2 a_{j}+k_{j}+k_{j}^{\prime}>2$, then $|j| \leq n_{3}^{*}$, we are in Case. 1.1. Hence in what follows, we always assume

$$
2 a_{j}+k_{j}+k_{j}^{\prime} \leq 2
$$

which implies

$$
k_{j}+k_{j}^{\prime} \leq 2 .
$$

From (4.15) and in view of $j \in\left\{n_{1}, n_{2}\right\}$, it follows that

$$
\begin{aligned}
I \leq & 2 \sum_{a, k, k^{\prime}}\left(K_{n_{1}}+K_{n_{1}}^{\prime}+K_{n_{2}}+K_{n_{2}}^{\prime}\right) \\
& \times e^{-\left(2-2^{\theta}\right) \delta_{1} \sum_{i \geq 3}\left(n_{i}^{*}\right)^{\theta}-\left(2-2^{\theta}\right) \delta_{2} \sum_{i \geq 3}\left(N_{i}^{*}\right)^{\theta} .}
\end{aligned}
$$

In view of (4.4) and (4.11), we have

$$
\sum_{n}\left(2 \alpha_{n}+\kappa_{n}+\kappa_{n}^{\prime}\right) \leq 2 \sum_{i \geq 3}\left(n_{i}^{*}\right)^{\theta}+2 \sum_{i \geq 3}\left(N_{j}^{*}\right)^{\theta} .
$$

Moreover, note that $\forall j$,

$$
K_{j}+K_{j}^{\prime} \leq \kappa_{j}+\kappa_{j}^{\prime}-k_{j}-k_{j}^{\prime}+2 \leq \kappa_{j}+\kappa_{j}^{\prime}+2 .
$$

Hence,

$$
\begin{aligned}
I \leq & 2 \sum_{a, k, k^{\prime}}\left(\kappa_{n_{1}}+\kappa_{n_{1}}^{\prime}+\kappa_{n_{2}}+\kappa_{n_{2}}^{\prime}+4\right) \\
& \times e^{-\left(2-2^{\theta}\right) \delta_{1} \sum_{i \geq 3}\left(n_{i}^{*}\right)^{\theta}-\left(2-2^{\theta}\right) \delta_{2} \sum_{i \geq 3}\left(N_{i}^{*}\right)^{\theta}} \\
\leq & 2 \sum_{a, k, k^{\prime}}\left(\kappa_{n_{1}}+\kappa_{n_{1}}^{\prime}+\kappa_{n_{2}}+\kappa_{n_{2}}^{\prime}+4\right) \\
& \times e^{-\frac{1}{2}\left(2-2^{\theta}\right) \delta_{1} \sum_{i \geq 3}\left(n_{i}^{*}\right)^{\theta}} e^{-\frac{1}{2}\left(2-2^{\theta}\right)\left(\delta_{2} \sum_{i \geq 3}\left(N_{i}^{*}\right)^{\theta}+\delta_{1} \sum_{i \geq 3}\left(n_{i}^{*}\right)^{\theta}\right)} \\
\leq & 2 \sum_{a, k, k^{\prime}}\left(\kappa_{n_{1}}+\kappa_{n_{1}}^{\prime}+\kappa_{n_{2}}+\kappa_{n_{2}}^{\prime}+4\right) \\
& \times e^{-\frac{1}{2}\left(2-2^{\theta}\right) \delta_{1} \sum_{i \geq 3}\left(n_{i}^{*}\right)^{\theta}} e^{-\frac{1}{4}\left(\delta_{1} \wedge \delta_{2}\right)\left(2-2^{\theta}\right) \sum_{n}\left(2 \alpha_{n}+\kappa_{n}+\kappa_{n}^{\prime}\right)},
\end{aligned}
$$

where the last inequality is based on (4.16) and $\delta_{1} \wedge \delta_{2}=\min \left\{\delta_{1}, \delta_{2}\right\}$.

Remark 4.4. Obviously, $\left\{n_{1}, n_{2}\right\} \cap \operatorname{supp} \mathcal{M}_{\alpha \kappa \kappa^{\prime}} \neq \emptyset$, and if $\left\{n_{i}\right\}_{i \geq 3}$ and $n_{1}$ (resp. $n_{2}$ ) is specified, then $n_{2}$ (resp. $n_{1}$ ) is determined uniquely. Thus $n_{1}, n_{2}$ range in a set of cardinality no more than

$$
\# \operatorname{supp} \mathcal{M}_{\alpha \kappa \kappa^{\prime}} \leq \sum_{n}\left(2 \alpha_{n}+\kappa_{n}+\kappa_{n}^{\prime}\right) \text {. }
$$

Also, if $\left\{n_{i}\right\}_{i \geq 1}$ is given, then $\left\{2 a_{n}+k_{n}+k_{n}^{\prime}\right\}_{n \in \mathbb{Z}}$ is specified, and hence $\left(a, k, k^{\prime}\right)$ is specified up to a factor of

$$
\prod_{n}\left(1+l_{n}^{2}\right)
$$

where

$$
l_{n}=\#\left\{j: n_{j}=n\right\} .
$$


Following the inequality (4.17), we thus obtain

$$
\begin{aligned}
I \leq & 2 \sum_{\left\{n_{i}\right\}_{i \geq 1}} \prod_{m}\left(1+l_{m}^{2}\right)\left(\kappa_{n_{1}}+\kappa_{n_{1}}^{\prime}+\kappa_{n_{2}}+\kappa_{n_{2}}^{\prime}+4\right) \\
& \times e^{-\frac{1}{2}\left(2-2^{\theta}\right) \delta_{1} \sum_{i \geq 3}\left(n_{i}^{*}\right)^{\theta}} e^{-\frac{1}{4}\left(\delta_{1} \wedge \delta_{2}\right)\left(2-2^{\theta}\right) \sum_{n}\left(2 \alpha_{n}+\kappa_{n}+\kappa_{n}^{\prime}\right)} \\
= & 2 \sum_{\left\{n_{i}\right\}_{i \geq 3}} \prod_{m}\left(1+l_{m}^{2}\right)\left(\sum_{n_{1}, n_{2}}\left(\kappa_{n_{1}}+\kappa_{n_{1}}^{\prime}+\kappa_{n_{2}}+\kappa_{n_{2}}^{\prime}+4\right)\right) \\
& \times e^{-\frac{1}{2}\left(2-2^{\theta}\right) \delta_{1} \sum_{i \geq 3}\left(n_{i}^{*}\right)^{\theta}} e^{-\frac{1}{4}\left(\delta_{1} \wedge \delta_{2}\right)\left(2-2^{\theta}\right) \sum_{n}\left(2 \alpha_{n}+\kappa_{n}+\kappa_{n}^{\prime}\right)} \\
\leq & 2 \sum_{\left\{n_{i}\right\}_{i \geq 3}} \prod_{m}\left(1+l_{m}^{2}\right)\left(\sum_{n}\left(\kappa_{n}+\kappa_{n}^{\prime}\right)+4 \# \operatorname{supp} \mathcal{M}_{\alpha \kappa \kappa^{\prime}}\right) \\
& \times e^{-\frac{1}{2}\left(2-2^{\theta}\right) \delta_{1} \sum_{i \geq 3}\left(n_{i}^{*}\right)^{\theta}} e^{-\frac{1}{4}\left(\delta_{1} \wedge \delta_{2}\right)\left(2-2^{\theta}\right) \sum_{n}\left(2 \alpha_{n}+\kappa_{n}+\kappa_{n}^{\prime}\right)}
\end{aligned}
$$

(the inequality is based on Remark 4.4)

$$
\begin{aligned}
\leq & 10 \sum_{\left\{n_{i}\right\}_{i \geq 3}} \prod_{m}\left(1+l_{m}^{2}\right) e^{-\frac{1}{2}\left(2-2^{\theta}\right) \delta_{1} \sum_{i \geq 3}\left(n_{i}^{*}\right)^{\theta}} \\
& \times\left(\sum_{n}\left(2 \alpha_{n}+\kappa_{n}+\kappa_{n}^{\prime}\right)\right) e^{-\frac{1}{4}\left(\delta_{1} \wedge \delta_{2}\right)\left(2-2^{\theta}\right) \sum_{n}\left(2 \alpha_{n}+\kappa_{n}+\kappa_{n}^{\prime}\right)}
\end{aligned}
$$

(based on 4.18) )

$\leq \frac{C_{3}(\theta)}{\delta_{1} \wedge \delta_{2}} \sum_{\left\{n_{i}\right\}_{i \geq 3}} \prod_{|m| \leq\left|n_{1}\right|}\left(1+l_{m}^{2}\right) e^{-\frac{1}{2}\left(2-2^{\theta}\right) \delta_{1} \sum_{i \geq 3}\left(n_{i}^{*}\right)^{\theta}}$

(in view of (7.24) )

$$
\begin{aligned}
= & \frac{C_{3}(\theta)}{\delta_{1} \wedge \delta_{2}} \sum_{\left\{l_{m}\right\}_{|m| \leq\left|n_{3}\right|}}\left(\prod_{|m| \leq\left|n_{1}\right|}\left(1+l_{m}^{2}\right) e^{-\frac{1}{6}\left(2-2^{\theta}\right) \delta_{1} \sum_{|m| \leq\left|n_{3}\right|} l_{m}|m|^{\theta}}\right) \\
& \times e^{-\frac{1}{3}\left(2-2^{\theta}\right) \delta_{1} \sum_{|m| \leq\left|n_{3}\right|} l_{m}|m|^{\theta}} \\
\leq & \frac{C_{3}(\theta)}{\delta_{1} \wedge \delta_{2}} \sup _{\left\{l_{m}\right\}_{|m| \leq\left|n_{3}\right|}}\left(\prod_{|m| \leq\left|n_{1}\right|}\left(1+l_{m}^{2}\right) e^{-\frac{1}{6}\left(2-2^{\theta}\right) \delta_{1} \sum_{|m| \leq\left|n_{3}\right|} l_{m}|m|^{\theta}}\right) \\
& \times \sum_{\left\{l_{m}\right\}_{|m| \leq\left|n_{3}\right|}} e^{-\frac{1}{3}\left(2-2^{\theta}\right) \delta_{1} \sum_{|m| \leq\left|n_{3}\right|} l_{m}|m|^{\theta}} \\
\leq & \frac{C_{3}(\theta)}{\delta_{1} \wedge \delta_{2}}\left(\frac{1}{\delta_{1}}\right)^{C_{4}(\theta) \delta_{1}^{-\frac{1}{\theta}}} \sum_{\left\{l_{m}\right\}_{|m| \leq\left|n_{3}\right|}} e^{-\frac{1}{3}\left(2-2^{\theta}\right) \delta_{1} \sum_{|m| \leq\left|n_{3}\right|} l_{m}|m|^{\theta}}
\end{aligned}
$$

(in view of (7.29) )

$\leq \frac{C_{3}(\theta)}{\delta_{1} \wedge \delta_{2}}\left(\frac{1}{\delta_{1}}\right)^{C_{4}(\theta) \delta_{1}^{-\frac{1}{\theta}}} \prod_{m \in \mathbb{Z}} \frac{1}{1-e^{-\frac{1}{3}\left(2-2^{\theta}\right) \delta_{1}|m|^{\theta}}}$

(in view of (7.12)

$\leq \frac{1}{\delta_{2}}\left(\frac{1}{\delta_{1}}\right)^{C(\theta) \delta_{1}^{-\frac{1}{\theta}}} \quad$ in view of $\left(\underline{7.16)}\right.$ and $0<\delta_{1} \ll 1$ ), 
THE STABILITY OF FULL DIMENSIONAL KAM TORI FOR NONLINEAR SCHRÖDINGER EQUATIOIY

where $C(\theta)$ is a positive constant depending on $\theta$ only.

Case. 2. $\nu_{1}^{*}>N_{1}^{*}$.

In view of (4.9), one has $n_{1}^{*}=\nu_{1}^{*}$. Hence, $n_{2}$ is determined by $n_{1}$ and $\left\{n_{i}\right\}_{i \geq 3}$. Similar as Case 1.2, we have

$$
\begin{aligned}
I & \leq \frac{C^{\prime}(\theta)}{\delta_{2}} \sum_{\left\{n_{i}\right\}_{i \geq 3}} e^{-\frac{1}{2}\left(2-2^{\theta}\right) \delta_{1} \sum_{i \geq 3}\left(n_{i}^{*}\right)^{\theta}} \prod_{m}\left(1+l_{m}^{2}\right) \\
& \leq \frac{1}{\delta_{2}}\left(\frac{1}{\delta_{1}}\right)^{C(\theta) \delta_{1}^{-\frac{1}{\theta}}}
\end{aligned}
$$

where $C^{\prime}(\theta)$ is some positive constant depending on $\theta$ only.

Next, we will estimate the symplectic transformation $\Phi_{F}$ induced by the Hamiltonian function $F$. Actually, we have

Lemma 4.5. Let $\theta \in(0,1), \rho>0$ and $0<\delta_{1}, \delta_{2} \ll 1$ (depending on $\theta, \rho$ ). Assume further

$$
\frac{1}{\delta_{2}}\left(\frac{1}{\delta_{1}}\right)^{C(\theta) \delta_{1}^{-\frac{1}{\theta}}}\|F\|_{\rho-\delta_{1}} \ll 1,
$$

where $C(\theta)$ is the constant given in 4.2) in Lemma 4.1. Then for any Hamiltonian function $H$, we get

$$
\left\|H \circ \Phi_{F}\right\|_{\rho} \leq\left(1+\frac{1}{\delta_{2}}\left(\frac{1}{\delta_{1}}\right)^{C_{1}(\theta) \delta_{1}^{-\frac{1}{\theta}}}\|F\|_{\rho-\delta_{1}}\right)\|H\|_{\rho-\delta_{2}},
$$

where $C_{1}(\theta)$ is a positive constant depending only on $\theta$.

Proof. Firstly, we expand $H \circ \Phi_{F}$ into the Taylor series

$$
H \circ \Phi_{F}=\sum_{n \geq 0} \frac{1}{n !} H^{(n)}
$$

where $H^{(n)}=\left\{H^{(n-1)}, F\right\}$ and $H^{(0)}=H$.

We will estimate $\left\|H^{(n)}\right\|_{\rho}$ by using Lemma 4.1 again and again:

$$
\begin{aligned}
\left\|H^{(n)}\right\|_{\rho}= & \left\|\left\{H^{(n-1)}, F\right\}\right\|_{\rho} \\
\leq & \left(\left(\frac{1}{\delta_{1}}\right)^{C(\theta) \delta_{1}^{-\frac{1}{\theta}}}\|F\|_{\rho-\delta_{1}}\right)\left(\frac{n}{\delta_{2}}\right)\left\|H^{(n-1)}\right\|_{\rho-\frac{\delta_{2}}{n}} \\
\leq & \left(\left(\frac{1}{\delta_{1}}\right)^{C(\theta) \delta_{1}^{-\frac{1}{\theta}}}\|F\|_{\rho-\delta_{1}}\right)^{2}\left(\frac{n}{\delta_{2}}\right)^{2}\left\|H^{(n-2)}\right\|_{\rho-\frac{2 \delta_{2}}{n}} \\
& \cdots\left(\left(\frac{1}{\delta_{1}}\right)^{C(\theta) \delta_{1}^{-\frac{1}{\theta}}}\|F\|_{\rho-\delta_{1}}\right)^{n}\left(\frac{n}{\delta_{2}}\right)^{n}\|H\|_{\rho-\delta_{2} .}
\end{aligned}
$$


Hence in view of 4.21, one has

$$
\begin{aligned}
& \left\|H \circ \Phi_{F}\right\|_{\rho} \leq \sum_{n \geq 0} \frac{1}{n !}\left(\left(\frac{1}{\delta_{1}}\right)^{C(\theta) \delta_{1}^{-\frac{1}{\theta}}}\|F\|_{\rho-\delta_{1}}\right)^{n}\left(\frac{n}{\delta_{2}}\right)^{n}\|H\|_{\rho-\delta_{2}} \\
& =\sum_{n \geq 0} \frac{n^{n}}{n !}\left(\frac{1}{\delta_{2}}\left(\frac{1}{\delta_{1}}\right)^{C(\theta) \delta_{1}^{-\frac{1}{\theta}}}\|F\|_{\rho-\delta_{1}}\right)^{n}\|H\|_{\rho-\delta_{2}} \\
& \leq \sum_{n \geq 0}\left(\frac{e}{\delta_{2}}\left(\frac{1}{\delta_{1}}\right)^{C(\theta) \delta_{1}^{-\frac{1}{\theta}}}\|F\|_{\rho-\delta_{1}}\right)^{n}\|H\|_{\rho-\delta_{2}} \\
& \text { (in view of } n^{n}<n ! e^{n} \text { ) } \\
& \leq\left(1+\frac{1}{\delta_{2}}\left(\frac{1}{\delta_{1}}\right)^{C(\theta) \delta_{1}^{-\frac{1}{\theta}}}\|F\|_{\rho-\delta_{1}}\right)\|H\|_{\rho-\delta_{2}} \\
& \text { (in view of (4.19) and } 0<\delta_{1} \ll 1 \text { ), }
\end{aligned}
$$

where $C_{1}(\theta)$ is a positive constant depending on $\theta$ only.

4.2. The new perturbation $R_{+}$and the new normal form $N_{+}$. Firstly, for $i=0,1$, one has

$$
\begin{aligned}
\left\|F_{i}\right\|_{\rho+\delta} & \leq \frac{C_{1}(\theta)}{\delta^{2}}\left\|F_{i}\right\|_{\rho+\frac{\delta}{2}}^{+} & & (\text {in view of }[\text { (3.17) }) \\
& \leq \frac{C_{1}(\theta)}{\delta^{2}} \cdot \frac{e^{3}}{\gamma} \cdot e^{C_{2}(\theta) \delta^{-\frac{5}{\theta}}}\left\|R_{i}\right\|_{\rho}^{+} & & (\text {in view of }(\underline{3.18})) \\
& \leq \frac{1}{\gamma \delta^{3}} e^{\delta^{-\frac{6}{\theta}}}\left\|R_{i}\right\|_{\rho}^{+} & & \text {(by assuming } \delta \ll 1) .
\end{aligned}
$$

Recall the new term $R_{+}$is given by (3.11), i.e. $R_{+}=R_{2}+\mathcal{R}$, where

$$
\mathcal{R}=\int_{0}^{1}\left\{(1-t)\{N, F\}+R_{0}+R_{1}, F\right\} \circ X_{F}^{t} d t+\int_{0}^{1}\left\{R_{2}, F\right\} \circ X_{F}^{t} d t .
$$

Write

$$
R_{+}=R_{0+}+R_{1+}+R_{2+}
$$

and write $\mathcal{R}$ as

$$
\begin{aligned}
\mathcal{R}= & \int_{0}^{1}\left\{R_{0}, F\right\} \circ X_{F}^{t} d t \\
& +\int_{0}^{1}\left\{R_{1}, F\right\} \circ X_{F}^{t} d t \\
& +\int_{0}^{1}\left\{R_{2}, F\right\} \circ X_{F}^{t} d t \\
& +\int_{0}^{1}(1-t)\{\{N, F\}, F\} \circ X_{F}^{t} d t .
\end{aligned}
$$


THE STABILITY OF FULL DIMENSIONAL KAM TORI FOR NONLINEAR SCHRÖDINGER EQUATIOII

Firstly note that the term (4.25) contributes to $R_{0+}, R_{1+}, R_{2+}$ and we get

$$
\begin{aligned}
\left\|\int_{0}^{1}\left\{R_{0}, F\right\} \circ X_{F}^{t} d t\right\|_{\rho+2 \delta} & =\|\sum_{n \geq 1} \frac{1}{n !} \underbrace{\left\{\cdots\left\{R_{0}, F\right\}, \cdots, F\right\}}_{n-\text { fold }}\|_{\rho+2 \delta} \\
& \leq \frac{1}{\delta} \cdot\left(\frac{1}{\delta}\right)^{C_{1}(\theta) \delta^{-\frac{1}{\theta}}}\|F\|_{\rho+\delta}\left\|R_{0}\right\|_{\rho+\delta}
\end{aligned}
$$

(following the proof of Lemma 4.5)

$$
\begin{aligned}
\leq & \frac{1}{\delta}\left(\frac{1}{\delta}\right)^{C_{1}(\theta) \delta^{-\frac{1}{\theta}}} \cdot \frac{1}{\gamma \delta^{3}} e^{\delta^{-\frac{6}{\theta}}} \cdot \frac{C_{2}(\theta)}{\delta^{2}}\left(\left\|R_{0}\right\|_{\rho}^{+}+\left\|R_{1}\right\|_{\rho}^{+}\right)\left\|R_{0}\right\|_{\rho}^{+} \\
& (\text {by }(\underline{3.17)} \text { and }(4.23)) \\
\leq & \frac{1}{\gamma} \cdot e^{\delta^{-\frac{8}{\theta}}}\left\|R_{0}\right\|_{\rho}^{+}\left(\left\|R_{0}\right\|_{\rho}^{+}+\left\|R_{1}\right\|_{\rho}^{+}\right),
\end{aligned}
$$

and consequently

$$
\begin{aligned}
\left\|\int_{0}^{1}\left\{R_{0}, F\right\} \circ X_{F}^{t} d t\right\|_{\rho+3 \delta}^{+} & \leq\left(\frac{1}{\delta}\right)^{C(\theta) \delta^{-\frac{1}{\theta}}}\left\|\int_{0}^{1}\left\{R_{0}, F\right\} \circ X_{F}^{t} d t\right\|_{\rho+2 \delta} \quad(\text { from (3.16) }) \\
& \left.\leq\left(\frac{1}{\delta}\right)^{C(\theta) \delta^{-\frac{1}{\theta}}} \cdot \frac{1}{\gamma} \cdot e^{\delta^{-\frac{8}{\theta}}}\left\|R_{0}\right\|_{\rho}^{+}\left(\left\|R_{0}\right\|_{\rho}^{+}+\left\|R_{1}\right\|_{\rho}^{+}\right) \quad \text { (from (4.28) }\right) \\
(4.29) & \leq \frac{1}{\gamma} \cdot e^{\delta^{-\frac{10}{\theta}}}\left\|R_{0}\right\|_{\rho}^{+}\left(\left\|R_{0}\right\|_{\rho}^{+}+\left\|R_{1}\right\|_{\rho}^{+}\right) .
\end{aligned}
$$

Secondly, we consider the term (4.25) and write

$$
\begin{aligned}
\text { (4.25) }= & \sum_{n \geq 1} \frac{1}{n !} \underbrace{\left\{\cdots\left\{R_{1}, F\right\}, F, \cdots, F\right\}}_{n-\text { fold }} \\
= & \sum_{n \geq 1} \frac{1}{n !} \underbrace{\left\{\cdots\left\{R_{1}, F_{0}\right\}, F, \cdots, F\right\}}_{(n-1)-\text { fold }} \\
& +\left\{R_{1}, F_{1}\right\} \\
& +\sum_{n \geq 2} \frac{1}{n !} \underbrace{\left\{\cdots\left\{R_{1}, F_{1}\right\}, F, \cdots, F\right\}}_{(n-1)-\text { fold }} .
\end{aligned}
$$

Note that (4.30) contributes to $R_{0+}, R_{1+}, R_{2+}$, (4.31) contributes to $R_{1+}, R_{2+}$ and (4.32) contributes to $R_{0+}, R_{1+}, R_{2+}$.

Moreover, following the proof of (4.29), one has

$$
\begin{aligned}
& \|\left(\sqrt[4.300]{ }\left\|_{\rho+3 \delta}^{+} \leq \frac{1}{\gamma} \cdot e^{\delta^{-\frac{10}{\theta}}}\right\| R_{1}\left\|_{\rho}^{+}\right\| R_{0} \|_{\rho}^{+}\left(\left\|R_{0}\right\|_{\rho}^{+}+\left\|R_{1}\right\|_{\rho}^{+}\right),\right. \\
& \|(4.31)\|_{\rho+3 \delta}^{+} \leq \frac{1}{\gamma} \cdot e^{\delta^{-\frac{10}{\theta}}}\left\|R_{1}\right\|_{\rho}^{+}\left\|R_{1}\right\|_{\rho}^{+}, \\
& \|(4.32)\|_{\rho+3 \delta}^{+} \leq \frac{1}{\gamma} \cdot e^{\delta^{-\frac{10}{\theta}}}\left\|R_{1}\right\|_{\rho}^{+}\left\|R_{1}\right\|_{\rho}^{+}\left(\left\|R_{0}\right\|_{\rho}^{+}+\left\|R_{1}\right\|_{\rho}^{+}\right) .
\end{aligned}
$$


Thirdly, we consider the term (4.26) and write

$$
\begin{aligned}
\text { (4.26) }= & \sum_{n \geq 1} \frac{1}{n !} \underbrace{\left\{\cdots\left\{R_{2}, F\right\}, F, \cdots, F\right\}}_{n-\text { fold }} \\
= & \left\{R_{2}, F_{0}\right\} \\
& +\left\{R_{2}, F_{1}\right\} \\
& +\sum_{n \geq 2} \frac{1}{n !} \underbrace{\left\{\cdots\left\{R_{2}, F_{0}\right\}, F, \cdots, F\right\}}_{(n-1)-\text { fold }} \\
& +\left\{\left\{R_{1}, F_{1}\right\}, F\right\} \\
& +\sum_{n \geq 3} \frac{1}{n !} \underbrace{\left\{\cdots\left\{R_{2}, F_{1}\right\}, F, \cdots, F\right\}}_{(n-1)-\text { fold }} .
\end{aligned}
$$

Note that (4.36) contributes to $R_{1+}, R_{2+}$, (4.37) contributes to $R_{2+}$, (4.38) contributes to $R_{0+}, R_{1+}, R_{2+}$, (4.39) contributes to $R_{1+}, R_{2+}$ and (4.40) contributes to $R_{0+}, R_{1+}, R_{2+}$.

Similarly, one has

$$
\begin{aligned}
& \|(4.36)\|_{\rho+3 \delta}^{+} \leq \frac{1}{\gamma} \cdot e^{\delta^{-\frac{10}{\theta}}}\left\|R_{0}\right\|_{\rho}^{+}\left\|R_{2}\right\|_{\rho}^{+}, \\
& \| \text {(4.37) }\left\|_{\rho+3 \delta}^{+} \leq \frac{1}{\gamma} \cdot e^{\delta^{-\frac{10}{\theta}}}\right\| R_{2}\left\|_{\rho}^{+}\right\| R_{1} \|_{\rho}^{+}, \\
& \|(4.38)\|_{\rho+3 \delta}^{+} \leq \frac{1}{\gamma} \cdot e^{\delta^{-\frac{10}{\theta}}}\left\|R_{0}\right\|_{\rho}^{+}\left\|R_{2}\right\|_{\rho}^{+}\left(\left\|R_{0}\right\|_{\rho}^{+}+\left\|R_{1}\right\|_{\rho}^{+}\right), \\
& \|(4.39)\|_{\rho+3 \delta}^{+} \leq \frac{1}{\gamma} \cdot e^{\delta^{-\frac{10}{\theta}}}\left\|R_{2}\right\|_{\rho}^{+}\left\|R_{1}\right\|_{\rho}^{+}\left(\left\|R_{0}\right\|_{\rho}^{+}+\left\|R_{1}\right\|_{\rho}^{+}\right), \\
& \| \text {(4.400) }\left\|_{\rho+3 \delta}^{+} \leq \frac{1}{\gamma} \cdot e^{\delta^{-\frac{10}{\theta}}}\right\| R_{2}\left\|_{\rho}^{+}\right\| R_{1} \|_{\rho}^{+}\left(\left\|R_{0}\right\|_{\rho}^{+}+\left\|R_{1}\right\|_{\rho}^{+}\right)^{2} \text {. }
\end{aligned}
$$

Finally, we consider the term (4.27) and write

$$
\begin{aligned}
\text { (4.27) }= & \sum_{n \geq 2} \frac{1}{n !} \underbrace{\{\cdots\{N, F\}, F, \cdots, F\}}_{n-\text { fold }} \\
= & \sum_{n \geq 2} \frac{1}{n !} \underbrace{\left\{\cdots\left\{-R_{0}-R_{1}+\left[R_{0}\right]+\left[R_{1}\right]\right\}, F, \cdots, F\right\}}_{(n-1)-\text { fold }} \\
& (\text { in view of (33.4)) } \\
= & \sum_{n \geq 2} \frac{1}{n !} \underbrace{\left\{\cdots\left\{-R_{0}+\left[R_{0}\right], F\right\}, F, \cdots, F\right\}}_{(n-1)-\text { fold }} \\
& +\sum_{n \geq 2} \frac{1}{n !} \underbrace{\left\{\cdots\left\{-R_{1}+\left[R_{1}\right], F_{0}\right\}, F, \cdots, F\right\}}_{(n-2)-\text { fold }} \\
& +\left\{-R_{1}+\left[R_{1}\right], F_{1}\right\} \\
& +\sum_{n \geq 3} \frac{1}{n !} \underbrace{\left\{\cdots\left\{-R_{1}+\left[R_{1}\right], F_{1}\right\}, F, \cdots, F\right\}}_{(n-1)-\text { fold }} .
\end{aligned}
$$


THE STABILITY OF FULL DIMENSIONAL KAM TORI FOR NONLINEAR SCHRÖDINGER EQUATIOR

Note that (4.46) contributes to $R_{0+}, R_{1+}, R_{2+}$, (4.47) contributes to $R_{0+}, R_{1+}, R_{2+}$, (4.48) contributes to $R_{1+}, R_{2+}$ and (4.49) contributes to $R_{0+}, R_{1+}, R_{2+}$.

Moreover, one has

$$
\begin{aligned}
& \|(4.46)\|_{\rho+3 \delta}^{+} \leq \frac{1}{\gamma} \cdot e^{\delta^{-\frac{10}{\theta}}}\left\|R_{0}\right\|_{\rho}^{+}\left(\left\|R_{0}\right\|_{\rho}^{+}+\left\|R_{1}\right\|_{\rho}^{+}\right), \\
& \|(4.47)\|_{\rho+3 \delta}^{+} \leq \frac{1}{\gamma} \cdot e^{\delta^{-\frac{10}{\theta}}}\left\|R_{1}\right\|_{\rho}^{+}\left\|R_{0}\right\|_{\rho}^{+}\left(\left\|R_{0}\right\|_{\rho}^{+}+\left\|R_{1}\right\|_{\rho}^{+}\right), \\
& \|(4.48)\|_{\rho+3 \delta}^{+} \leq \frac{1}{\gamma} \cdot e^{\delta^{-\frac{10}{\theta}}}\left\|R_{1}\right\|_{\rho}^{+}\left\|R_{1}\right\|_{\rho}^{+}, \\
& \|(4.49)\|_{\rho+3 \delta}^{+} \leq \frac{1}{\gamma} \cdot e^{\delta^{-\frac{10}{\theta}}}\left\|R_{1}\right\|_{\rho}^{+}\left\|R_{1}\right\|_{\rho}^{+}\left(\left\|R_{0}\right\|_{\rho}^{+}+\left\|R_{1}\right\|_{\rho}^{+}\right) .
\end{aligned}
$$

Consequently

$$
\begin{aligned}
\left\|R_{0+}\right\|_{\rho+3 \delta}^{+} & \leq \frac{1}{\gamma} \cdot e^{\delta^{-\frac{10}{\theta}}}\left(\left\|R_{0}\right\|_{\rho}^{+}+\left\|R_{1}\right\|_{\rho}^{+}\right)\left(\left\|R_{0}\right\|_{\rho}^{+}+\left\|R_{1}\right\|_{\rho}^{+2}\right), \\
\left\|R_{1+}\right\|_{\rho+3 \delta}^{+} & \leq \frac{1}{\gamma} \cdot e^{\delta^{-\frac{10}{\theta}}}\left(\left\|R_{0}\right\|_{\rho}^{+}+\left\|R_{1}\right\|_{\rho}^{+2}\right), \\
\left\|R_{2+}\right\|_{\rho+3 \delta}^{+} & \leq\left\|R_{2}\right\|_{\rho}^{+}+\frac{1}{\gamma} \cdot e^{\delta^{-\frac{10}{\theta}}}\left(\left\|R_{0}\right\|_{\rho}^{+}+\left\|R_{1}\right\|_{\rho}^{+}\right) .
\end{aligned}
$$

The new normal form $N_{+}$is given in (3.10). Note that $\left[R_{0}\right]$ (in view of (3.5)) is a constant which does not affect the Hamiltonian vector field. Moreover, in view of (3.6), we denote by

$$
\omega_{n+}=n^{2}+\widetilde{V}_{n}+\sum_{a} B_{a 00}^{(n)} \mathcal{M}_{a 00}
$$

where the terms $\sum_{a} B_{a 00}^{(n)} \mathcal{M}_{a 00}$ is the so-called frequency shift. The estimate of $\left|\sum_{a} B_{a 00}^{(n)} \mathcal{M}_{a 00}\right|$ will be given in the next section (see (5.33) for the details).

\section{Iteration And CONVERGence}

Now we give the precise set-up of iteration parameters. Let $s \geq 1$ be the $s$-th KAM step.

$$
\begin{aligned}
& \delta_{s}=\frac{\rho}{s^{2}} \\
& \rho_{s+1}=\rho_{s}+3 \delta_{s}, \\
& \epsilon_{s}=\epsilon_{0}^{\left(\frac{3}{2}\right)^{s}}, \text { which dominates the size of the perturbation, } \\
& \lambda_{s}=e^{-C(\theta)\left(\ln \frac{1}{\epsilon_{s+1}}\right)^{\frac{4}{\theta+4}}} \\
& \eta_{s+1}=\frac{1}{20} \lambda_{s} \eta_{s} \\
& d_{0}=0, d_{s+1}=d_{s}+\frac{1}{\pi^{2}(s+1)^{2}}, \\
& D_{s}=\left\{\left(q_{n}\right)_{n \in \mathbb{Z}}: \frac{1}{2}+d_{s} \leq\left|q_{n}\right| e^{r|n|^{\theta}} \leq 1-d_{s}\right\}
\end{aligned}
$$

Denote the complex cube of size $\lambda>0$ :

$$
\mathcal{C}_{\lambda}(\widetilde{V})=\left\{\left(V_{n}\right)_{n} \in \prod_{n \in \mathbb{Z}} \mathbb{C}:\left|V_{n}-\widetilde{V}_{n}\right| \leq \lambda\right\}
$$

Lemma 5.1. Suppose $H_{s}=N_{s}+R_{s}$ is real analytic on $D_{s} \times \mathcal{C}_{\eta_{s}}\left(V_{s}\right)$, where

$$
N_{s}=\sum_{n \in \mathbb{Z}}\left(n^{2}+\widetilde{V}_{n, s}\right)\left|q_{n}\right|^{2}
$$


is a normal form with coefficients satisfying

$$
\begin{aligned}
& \widetilde{V}_{s}\left(V_{s}\right)=\omega, \\
& \left\|\frac{\partial \widetilde{V}_{s}}{\partial V}-I\right\|_{l^{\infty} \rightarrow l^{\infty}}<d_{s} \epsilon_{0}^{\frac{1}{10}},
\end{aligned}
$$

and $R_{s}=R_{0, s}+R_{1, s}+R_{2, s}$ satisfying

$$
\begin{aligned}
& \left\|R_{0, s}\right\|_{\rho_{s}}^{+} \leq \epsilon_{s}, \\
& \left\|R_{1, s}\right\|_{\rho_{s}}^{+} \leq \epsilon_{s}^{0.6}, \\
& \left\|R_{2, s}\right\|_{\rho_{s}}^{+} \leq\left(1+d_{s}\right) \epsilon_{0} .
\end{aligned}
$$

Then for all $V \in \mathcal{C}_{\eta_{s}}\left(V_{s}\right)$ satisfying $\widetilde{V}_{s}(V) \in \mathcal{C}_{\lambda_{s}}(\omega)$, there exist real analytic symplectic coordinate transformations $\Phi_{s+1}: D_{s+1} \rightarrow D_{s}$ satisfying

$$
\begin{aligned}
& \left\|\Phi_{s+1}-i d\right\|_{r, \infty} \leq \epsilon_{s}^{0.5}, \\
& \left\|D \Phi_{s+1}-I\right\|_{(r, \infty) \rightarrow(r, \infty)} \leq \epsilon_{s}^{0.5},
\end{aligned}
$$

such that for $H_{s+1}=H_{s} \circ \Phi_{s+1}=N_{s+1}+R_{s+1}$, the same assumptions as above are satisfied with ' $s+1$ ' in place of ' $s$ ', where $\mathcal{C}_{\eta_{s+1}}\left(V_{s+1}\right) \subset \widetilde{V}_{s}^{-1}\left(\mathcal{C}_{\lambda_{s}}(\omega)\right)$ and

$$
\begin{gathered}
\left\|\widetilde{V}_{s+1}-\widetilde{V}_{s}\right\|_{\infty} \leq \epsilon_{s}^{0.5}, \\
\left\|V_{s+1}-V_{s}\right\|_{\infty} \leq 2 \epsilon_{s}^{0.5} .
\end{gathered}
$$

Proof. In the step $s \rightarrow s+1$, there is saving of a factor

$$
e^{-\delta_{s}\left(\sum_{n}\left(2 a_{n}+k_{n}+k_{n}^{\prime}\right)|n|^{\theta}-2\left|n_{1}^{*}\right|^{\theta}\right)} \leq e^{-\left(2-2^{\theta}\right) \delta_{s} \sum_{i \geq 3}\left|n_{i}\right|^{\theta}} .
$$

Recalling after this step, we need

$$
\begin{aligned}
\left\|R_{0, s+1}\right\|_{\rho_{s+1}}^{+} & \leq \epsilon_{s+1}, \\
\left\|R_{1, s+1}\right\|_{\rho_{s+1}}^{+} & \leq \epsilon_{s+1}^{0.6} .
\end{aligned}
$$

Consequently, in $R_{i, s}(i=0,1)$, it suffices to eliminate the nonresonant monomials $\mathcal{M}_{a k k^{\prime}}$ for which

that is

$$
e^{-\left(2-2^{\theta}\right) \delta_{s} \sum_{i \geq 3}\left|n_{i}\right|^{\theta}} \geq \epsilon_{s+1}
$$

$$
\sum_{i \geq 3}\left|n_{i}\right|^{\theta} \leq \frac{s^{2}}{\left(2-2^{\theta}\right) \rho} \ln \frac{1}{\epsilon_{s+1}} .
$$

On the other hand, in the small divisors analysis, we need only impose Diophantine conditions when (7.1) holds, which implies

$$
\begin{aligned}
\sum_{n \in \mathbb{Z}}\left|k_{n}-k_{n}^{\prime} \| n\right|^{\theta / 2} & \leq 3 \cdot 6^{\theta / 2} \sum_{i \geq 3}\left|n_{i}\right|^{\theta} \quad \text { (by Lemma 7.1) } \\
& \left.\leq \frac{3 \cdot 6^{\theta / 2} \cdot s^{2}}{\left(2-2^{\theta}\right) \rho} \ln \frac{1}{\epsilon_{s+1}} \text { (in view of (5.11) }\right) \\
& :=B .
\end{aligned}
$$

From (5.12), we need only impose condition on $\left(\widetilde{V}_{n}\right)_{|n| \leq N_{*}}$, where

$$
N_{*} \sim B^{2 / \theta} \text {. }
$$


THE STABILITY OF FULL DIMENSIONAL KAM TORI FOR NONLINEAR SCHRÖDINGER EQUATIO

Correspondingly, the Diophantine condition becomes

$$
\left\|\sum_{|n| \leq N_{*}}\left(k_{n}-k_{n}^{\prime}\right) \widetilde{V}_{n, s}\right\| \geq \gamma \prod_{|n| \leq N_{*}} \frac{1}{1+\left(k_{n}-k_{n}^{\prime}\right)^{2}|n|^{4}} .
$$

We finished the truncation step. Next we will show (5.14) preserves under small perturbation of $\left(\widetilde{V}_{n}\right)_{|n| \leq N_{*}}$ and this is equivalent to get lower bound on the right hand side of (5.14). More concretely, for large $N$ (will be specified latter), we have

$$
\begin{aligned}
\prod_{|n| \leq N_{*}} \frac{1}{1+\left(k_{n}-k_{n}^{\prime}\right)^{2}|n|^{4}} & =e^{\sum_{|n| \leq N} \ln \left(\frac{1}{1+\left(k_{n}-k_{n}^{\prime}\right)^{2}|n|^{4}}\right)+\sum_{|n|>N} \ln \left(\frac{1}{1+\left(k_{n}-k_{n}^{\prime}\right)^{2}|n|^{4}}\right)} \\
& \geq e^{-C(\theta) \sum_{|n| \leq N, k_{n} \neq k_{n}^{\prime}} \ln \left(\left|k_{n}-k_{n}^{\prime}\right||n|^{\frac{\theta}{2}}\right)-\sum_{|n|>N, k_{n} \neq k_{n}^{\prime}} 4\left(\left|k_{n}-k_{n}^{\prime}\right| \cdot \ln |n|\right)} \\
& \geq e^{-C(\theta) N \ln B-4 \sum_{|n|>N, k_{n} \neq k_{n}^{\prime}}\left(\left|k_{n}-k_{n}^{\prime}\right||n|^{\frac{\theta}{2}}\left(|n|^{-\frac{\theta}{2}} \ln |n|\right)\right)} \\
& \geq e^{\left.-C(\theta) N \ln B-C(\theta)\left(N^{-\frac{\theta}{2}} \ln N\right) B \quad \text { (again from (15.12) }\right)} \\
& \geq e^{-C(\theta) N \ln B-C(\theta) N^{-\frac{\theta}{2.5}} B} .
\end{aligned}
$$

Let

$$
N \sim\left(\frac{B}{\ln B}\right)^{\frac{2.5}{\theta+2.5}}
$$

then by (5.15), one has

$$
\begin{aligned}
\prod_{|n| \leq N_{*}} \frac{1}{1+\left(k_{n}-k_{n}^{\prime}\right)^{2}|n|^{4}} & \geq e^{-C(\theta) B^{\frac{2.5}{\theta+2.5}}(\ln B)^{\frac{-\theta}{\theta+2.5}}} \\
& \geq e^{-C(\theta) B^{\frac{3}{\theta+3}}} \\
& \geq e^{-C(\theta) s^{\frac{6}{3+\theta}}\left(\ln \frac{1}{\epsilon_{s+1}}\right)^{\frac{3}{\theta+3}}} \\
& >e^{-C(\theta)\left(\ln \frac{1}{\epsilon_{s+1}}\right)^{\frac{4}{\theta+4}}} \text { (for } \epsilon_{0} \text { sufficiently small). }
\end{aligned}
$$

Assuming $V \in \mathcal{C}_{\lambda_{s}}\left(\widetilde{V}_{s}\right)$, from the lower bound (5.16), the relation (5.14) remains true if we substitute $V$ for $\widetilde{V}_{s}$. Moreover, there is analyticity on $\mathcal{C}_{\lambda_{s}}\left(\widetilde{V}_{s}\right)$. The transformations $\Phi_{s+1}$ is obtained as the time- 1 map $\left.X_{F_{s}}^{t}\right|_{t=1}$ of the Hamiltonian vector field $X_{F_{s}}$ with $F_{s}=F_{0, s}+F_{1, s}$. Taking $\rho=\rho_{s}, \delta=\delta_{s}$ in Lemma 3.2, we get

$$
\left\|F_{i, s}\right\|_{\rho_{s}+\delta_{s}}^{+} \leq \frac{1}{\gamma} \cdot e^{C(\theta) \delta_{s}^{-\frac{5}{\theta}}}\left\|R_{i, s}\right\|_{\rho_{s}}^{+}
$$

where $i=0,1$. By Lemma 3.1, we get

$$
\left\|F_{i, s}\right\|_{\rho_{s}+2 \delta_{s}} \leq \frac{C(\theta)}{\delta_{s}^{2}}\left\|F_{i, s}\right\|_{\rho_{s}+\delta_{s}}^{+} .
$$

Combining (5.4), (5.5), (5.17) and (5.18), we get

$$
\left\|F_{s}\right\|_{\rho_{s}+2 \delta_{s}} \leq \frac{C(\theta)}{\gamma \delta_{s}^{2}} e^{C(\theta) \delta_{s}^{-\frac{5}{\theta}}}\left(\epsilon_{s}+\epsilon_{s}^{0.6}\right) .
$$


To estimate the norm of $X_{F_{s}}^{1}=\Phi_{s+1}$, we will prove a technical lemma which establishes a connection between the norm $\|\cdot\|_{\rho}$ and the sup-norm $\|\cdot\|_{\rho, \infty}$ firstly.

Lemma 5.2. Given a Hamiltonian

$$
H=\sum_{a, k, k^{\prime}} B_{a k k^{\prime}} \mathcal{M}_{a k k^{\prime}}
$$

then for any $r>7 \rho$, one has

$$
\sup _{\|q\|_{r, \infty}<1}\left\|X_{H}\right\|_{r, \infty} \leq C(\rho, \theta)\|H\|_{\rho},
$$

where $C(\rho, \theta)$ is a positive constant depending on $\rho$ and $\theta$ only.

Proof. In view of the definition (1.7), it suffices to estimate the upper bound for

$$
\left|\frac{\partial H}{\partial q_{j}} e^{r|j|^{\theta}}\right|
$$

for each $j \in \mathbb{Z}$. In view of (5.20), one has

$$
\frac{\partial H}{\partial q_{j}}=\sum_{a, k, k^{\prime}} B_{a k k^{\prime}}\left(\prod_{n \neq j} I_{n}(0)^{a_{n}} q_{n}^{k_{n}} \bar{q}_{n}^{k_{n}^{\prime}}\right)\left(k_{j} I_{j}(0)^{a_{j}} q_{j}^{k_{j}-1} \bar{q}_{j}^{k_{j}^{\prime}}\right)
$$

and moreover

$$
\left|\frac{\partial H}{\partial q_{j}} \cdot e^{r|j|^{\theta}}\right|=\left|e^{r|j|^{\theta}} \sum_{a, k, k^{\prime}} B_{a k k^{\prime}}\left(\prod_{n \neq j} I_{n}(0)^{a_{n}} q_{n}^{k_{n}} \bar{q}_{n}^{k_{n}^{\prime}}\right)\left(k_{j} I_{j}(0)^{a_{j}} q_{j}^{k_{j}-1} \bar{q}_{j}^{k_{j}^{\prime}}\right)\right| .
$$

Based on (2.12), one has

$$
\left|B_{a k k^{\prime}}\right| \leq\|H\|_{\rho} e^{\rho\left(\sum_{n}\left(2 a_{n}+k_{n}+k_{n}^{\prime}\right)|n|^{\theta}-2\left(n_{1}^{*}\right)^{\theta}\right)} .
$$

Therefore, in view of $\|q\|_{r, \infty}<1$ and (5.23), one has

$$
\begin{aligned}
|\underline{(5.22)}| & \leq\|H\|_{\rho}\left|e^{r|j|^{\theta}} \sum_{a, k, k^{\prime}} k_{j} e^{\rho\left(\sum_{n}\left(2 a_{n}+k_{n}+k_{n}^{\prime}\right)|n|^{\theta}-2\left(n_{1}^{*}\right)^{\theta}\right)} e^{-r\left(\sum_{n}\left(2 a_{n}+k_{n}+k_{n}^{\prime}\right)|n|^{\theta}-|j|^{\theta}\right)}\right| \\
& =\|H\|_{\rho}\left|\sum_{a, k, k^{\prime}} k_{j} e^{\rho\left(\sum_{n}\left(2 a_{n}+k_{n}+k_{n}^{\prime}\right)|n|^{\theta}-2\left(n_{1}^{*}\right)^{\theta}\right)} e^{-r\left(\sum_{n}\left(2 a_{n}+k_{n}+k_{n}^{\prime}\right)|n|^{\theta}-2|j|^{\theta}\right)}\right| .
\end{aligned}
$$

Now we will estimate the last inequality in the following two cases:

Case 1. $|j| \leq n_{3}^{*}$.

Then one has

$$
\begin{aligned}
& \left|\sum_{a, k, k^{\prime}} k_{j} e^{\rho\left(\sum_{n}\left(2 a_{n}+k_{n}+k_{n}^{\prime}\right)|n|^{\theta}-2\left(n_{1}^{*}\right)^{\theta}\right)} e^{-r\left(\sum_{n}\left(2 a_{n}+k_{n}+k_{n}^{\prime}\right)|n|^{\theta}-2|j|^{\theta}\right)}\right| \\
\leq & \left.\left|\sum_{a, k, k^{\prime}} k_{j} e^{\rho \sum_{i \geq 1}\left(n_{i}^{*}\right)^{\theta}} e^{-r\left(n_{1}^{*}\right)^{\theta}-r \sum_{i \geq 4}\left(n_{i}^{*}\right)^{\theta}}\right| \quad \text { in view of }|j| \leq n_{3}^{*}\right) \\
\leq & \sum_{a, k, k^{\prime}} k_{j} e^{\frac{1}{3}(-r+3 \rho) \sum_{i \geq 1}\left(n_{i}^{*}\right)^{\theta}} .
\end{aligned}
$$


THE STABILITY OF FULL DIMENSIONAL KAM TORI FOR NONLINEAR SCHRÖDINGER EQUATIO

Note that

$$
\sum_{i \geq 1}\left(n_{i}^{*}\right)^{\theta}=\sum_{n}\left(2 a_{n}+k_{n}+k_{n}^{\prime}\right)|n|^{\theta} \geq k_{j}
$$

Hence,

$$
\begin{aligned}
& \sum_{a, k, k^{\prime}} k_{j} e^{\frac{1}{3}(-r+3 \rho) \sum_{i \geq 1}\left(n_{i}^{*}\right)^{\theta}} \leq \sum_{a, k, k^{\prime}}\left(\sum_{i \geq 1}\left(n_{i}^{*}\right)^{\theta}\right) e^{\frac{1}{3}(-r+3 \rho) \sum_{i \geq 1}\left(n_{i}^{*}\right)^{\theta}}
\end{aligned}
$$

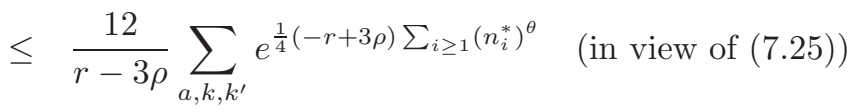

$$
\begin{aligned}
& =\frac{12}{r-3 \rho} \sum_{a, k, k^{\prime}} e^{\frac{1}{4}(-r+3 \rho) \sum_{n}\left(2 a_{n}+k_{n}+k_{n}^{\prime}\right)|n|^{\theta}} \\
& \leq \frac{12}{r-3 \rho}\left(\sum_{a} e^{\frac{1}{4}(-r+3 \rho) \sum_{n} 2 a_{n}|n|^{\theta}}\right)\left(\sum_{k} e^{\frac{1}{4}(-r+3 \rho) \sum_{n} k_{n}|n|^{\theta}}\right)^{2} \\
& \leq \frac{12}{r-3 \rho} \prod_{n \in \mathbb{Z}}\left(1-e^{\frac{1}{2}(-r+3 \rho)|n|^{\theta}}\right)^{-1} \prod_{n \in \mathbb{Z}}\left(1-e^{\frac{1}{4}(-r+3 \rho)|n|^{\theta}}\right)^{-2} \\
& \text { (in view of (7.12) } \\
& \leq \frac{12}{\rho} \prod_{n \in \mathbb{Z}}\left(1-e^{-\rho|n|^{\theta}}\right)^{-1} \prod_{n \in \mathbb{Z}}\left(1-e^{-\rho|n|^{\theta}}\right)^{-2} \\
& \text { (in view of } r>7 \rho \text { ) } \\
& \leq \frac{12}{\rho}\left(\frac{1}{\rho}\right)^{C_{1}(\theta) \rho^{-\frac{1}{\theta}}}
\end{aligned}
$$

where the last inequality is based on (7.16) and $C_{1}(\theta)$ is a positive constant depending on $\theta$ only.

Case 2. $|j|>n_{3}^{*}$, which implies $k_{j} \leq 2$.

Then one has

$$
\begin{aligned}
& \left|\sum_{a, k, k^{\prime}} k_{j} e^{\rho\left(\sum_{n}\left(2 a_{n}+k_{n}+k_{n}^{\prime}\right)|n|^{\theta}-2\left(n_{1}^{*}\right)^{\theta}\right)} e^{-r\left(\sum_{n}\left(2 a_{n}+k_{n}+k_{n}^{\prime}\right)|n|^{\theta}-2|j|^{\theta}\right)}\right| \\
\leq & \left.2\left|\sum_{a, k, k^{\prime}} e^{\rho \sum_{i \geq 3}\left(n_{i}^{*}\right)^{\theta}} e^{-r \sum_{i \geq 3}\left(n_{i}^{*}\right)^{\theta}}\right| \quad \text { in view of (2.8) and } k_{j} \leq 2\right) \\
(5.25)= & 2\left|\sum_{a, k, k^{\prime}} e^{(-r+\rho) \sum_{i \geq 3}\left(n_{i}^{*}\right)^{\theta}}\right| .
\end{aligned}
$$

If $\left\{n_{i}\right\}_{i \geq 1}$ is given, then $\left\{2 a_{n}+k_{n}+k_{n}^{\prime}\right\}_{n \in \mathbb{Z}}$ is specified, and hence $\left(a, k, k^{\prime}\right)$ is specified up to a factor of

$$
\prod_{n}\left(1+l_{n}^{2}\right)
$$

where

$$
l_{n}=\#\left\{j: n_{j}=n\right\} .
$$


Since $|j|>n_{3}^{*}$, then $j \in\left\{n_{1}, n_{2}\right\}$. Hence, if $\left(n_{i}\right)_{i \geq 3}$ and $j$ are given, then $n_{1}$ and $n_{2}$ are uniquely determined. Then, one has

$$
\begin{aligned}
& \text { (5.25) } \leq 2\left|\sum_{\left(n_{i}\right)_{i \geq 3}} \prod_{|n| \leq n_{1}^{*}}\left(1+l_{n}^{2}\right) e^{(-r+\rho) \sum_{i \geq 3}\left(n_{i}^{*}\right)^{\theta}}\right| \\
& \leq 10\left|\left(\sum_{\left(n_{i}\right)_{i \geq 3}} e^{-5 \rho \sum_{i \geq 3}\left(n_{i}^{*}\right)^{\theta}}\right) \sup _{\left(n_{i}\right)_{i \geq 3}}\left(\prod_{|n| \leq n_{1}^{*}}\left(1+l_{n}^{2}\right) e^{-\rho \sum_{i \geq 3}\left(n_{i}^{*}\right)^{\theta}}\right)\right| \\
& \left.\leq 10\left(\frac{1}{\rho}\right)^{C_{2}(\theta) \rho^{-\frac{1}{\theta}}} \sum_{\left(n_{i}\right)_{i \geq 3}} e^{-5 \rho \sum_{i \geq 3}\left(n_{i}^{*}\right)^{\theta}} \quad \text { (in view of (17.29) }\right) \\
& =10\left(\frac{1}{\rho}\right)^{C_{2}(\theta) \rho^{-\frac{1}{\theta}}} \sum_{\left(l_{n}\right)_{|n| \leq n_{3}^{*}}} e^{-5 \rho \sum_{|n| \leq n \frac{*}{3}} l_{n}|n|^{\theta}} \\
& \leq 10\left(\frac{1}{\rho}\right)^{C_{2}(\theta) \rho^{-\frac{1}{\theta}}} \prod_{n \in \mathbb{Z}}\left(1-e^{-5 \rho|n|^{\theta}}\right)^{-1} \quad \text { (in view of }(\sqrt{7.12}) \text { ) } \\
& \leq\left(\frac{1}{\rho}\right)^{C_{3}(\theta) \rho^{-\frac{1}{\theta}}} \quad \text { (in view of }(\sqrt{7.16}) \text { ), }
\end{aligned}
$$

where $C_{2}(\theta), C_{3}(\theta)$ are positive constants depending on $\theta$ only.

Hence, we finished the proof of (5.21).

By Lemma 5.2, we get

$$
\begin{aligned}
\sup _{\|q\|_{r, \infty}<1}\left\|X_{F_{s}}\right\|_{r, \infty} & \leq C(\rho, \theta)\left\|F_{s}\right\|_{\rho_{s}+2 \delta_{s}} \\
& \leq \frac{C(\rho, \theta)}{\gamma \delta_{s}^{2}} e^{C(\theta) \delta_{s}^{-\frac{5}{\theta}}}\left(\epsilon_{s}+\epsilon_{s}^{0.6}\right) \\
& \leq \epsilon_{s}^{0.55}
\end{aligned}
$$

where noting that $0<\epsilon_{0} \ll 1$ small enough and depending on $\rho, \theta$ only.

Since $\epsilon_{s}^{0.55} \ll \frac{1}{\pi^{2}(s+1)^{2}}=d_{s+1}-d_{s}$, we have $\Phi_{s+1}: D_{s+1} \rightarrow D_{s}$ with

$$
\left\|\Phi_{s+1}-i d\right\|_{r, \infty} \leq \sup _{q \in D_{s}}\left\|X_{F_{s}}\right\|_{r, \infty} \leq \epsilon_{s}^{0.55}<\epsilon_{s}^{0.5}
$$

which is the estimate (5.7). Moreover, from (5.26) we get

$$
\sup _{q \in D_{s}}\left\|D X_{F_{s}}-I\right\|_{r, \infty} \leq \frac{1}{d_{s}} \epsilon_{s}^{0.55} \ll \epsilon_{s}^{0.5}
$$

and thus the estimate (5.8) follows. 
THE STABILITY OF FULL DIMENSIONAL KAM TORI FOR NONLINEAR SCHRÖDINGER EQUATIO

Moreover, under the assumptions (5.4)-(5.6) at stage $s$, we get from (4.54), (4.55) and (4.56) that

$$
\begin{aligned}
& \left\|R_{0, s+1}\right\|_{\rho_{s+1}}^{+} \leq e^{\frac{3 s^{\frac{4}{\theta}+6}}{\tau^{\frac{2}{\theta}+3}}}\left(\epsilon_{0}^{\left(\frac{3}{2}\right)^{s}}+\epsilon_{0}^{0.9\left(\frac{3}{2}\right)^{s-1}}\right)\left(\epsilon_{0}^{\left(\frac{3}{2}\right)^{s}}+\epsilon_{0}^{1.8\left(\frac{3}{2}\right)^{s-1}}\right) \\
& =e^{\frac{3 s^{\frac{4}{\theta}+6}}{\tau^{\frac{2}{\theta}+3}}}\left(\epsilon_{0}^{2.2\left(\frac{3}{2}\right)^{s}}+\epsilon_{0}^{1.8\left(\frac{3}{2}\right)^{s}}+\epsilon_{0}^{1.6\left(\frac{3}{2}\right)^{s}}+\epsilon_{0}^{2\left(\frac{3}{2}\right)^{s}}\right) \\
& \leq 4 e^{\frac{3 s^{\frac{4}{\theta}+6}}{\tau^{\frac{2}{\theta}+3}}} \epsilon_{0}^{1.6\left(\frac{3}{2}\right)^{s}} \\
& <\epsilon_{0}^{1.5\left(\frac{3}{2}\right)^{s}} \text { for } 0<\epsilon_{0} \ll 1 \text { (depending on } \rho, \theta \text { only) } \\
& =\epsilon_{s+1} \text {, } \\
& \left\|R_{1, s+1}\right\|_{\rho_{s+1}}^{+} \leq e^{\frac{3 s^{\frac{4}{\theta}+6}}{\tau^{\frac{2}{\theta}+3}}}\left(\epsilon_{0}^{\left(\frac{3}{2}\right)^{s}}+\epsilon_{0}^{1.8\left(\frac{3}{2}\right)^{s-1}}\right) \\
& =e^{\frac{3 s^{\frac{4}{\theta}+6}}{\tau^{\frac{2}{\theta}+3}}}\left(\epsilon_{0}^{\left(\frac{3}{2}\right)^{s}}+\epsilon_{0}^{1.2\left(\frac{3}{2}\right)^{s}}\right) \\
& \leq 2 e^{\frac{3 s^{\frac{4}{\theta}}+6}{\tau^{\frac{2}{\theta}+3}}} \epsilon_{0}^{\left(\frac{3}{2}\right)^{s}} \\
& <\epsilon_{s+1}^{0.6} \text { for } 0<\epsilon_{0} \ll 1 \text { (depending on } \rho, \theta \text { only), }
\end{aligned}
$$

and

$$
\begin{aligned}
\left\|R_{2, s+1}\right\|_{\rho_{s+1}}^{+} & \leq\left\|R_{2, s}\right\|_{\rho_{s}}^{+}+e^{\frac{3 s^{\frac{4}{\theta}}+6}{\tau^{\frac{2}{\theta}+3}}}\left(\epsilon_{0}^{\left(\frac{3}{2}\right)^{s}}+\epsilon_{0}^{0.6\left(\frac{3}{2}\right)^{s}}\right) \\
& \leq\left(1+d_{s}\right) \epsilon_{0}+2 e^{\frac{3 s^{\frac{4}{\theta}}+6}{\tau^{\frac{2}{\theta}+3}}} \epsilon_{0}^{0.6\left(\frac{3}{2}\right)^{s}} \\
& \leq\left(1+d_{s+1}\right) \epsilon_{0} \text { for } 0<\epsilon_{0} \ll 1 \text { (depending on } \rho, \theta \text { only) }
\end{aligned}
$$

which are just the assumptions (5.4)-(5.6) at stage $s+1$.

Next, let $S=\mathcal{C}_{\frac{1}{10} \lambda_{s} \eta_{s}}\left(V_{s}\right)$ and if $V \in \mathcal{C}_{\frac{\eta_{s}}{2}}\left(V_{s}\right)$, by using Cauchy's estimate implies

$$
\begin{aligned}
\sum_{n \in \mathbb{Z}}\left|\frac{\partial \widetilde{V}_{m, s}}{\partial V_{n}}(V)\right| & \leq \frac{2}{\eta_{s}}\left\|\widetilde{V}_{s}\right\|_{\infty} \\
& \left.<10 \eta_{s}^{-1} \quad \text { (since }\left\|\widetilde{V}_{s}\right\|_{\infty} \leq 1\right) \text { for all } m,
\end{aligned}
$$

and let $X \in \mathcal{C}_{\frac{1}{10} \lambda_{s} \eta_{s}}\left(V_{s}\right)$, then

$$
\begin{aligned}
\left\|\widetilde{V}_{s}(X)-\omega\right\|_{\infty} & =\left\|\widetilde{V}_{s}(X)-\widetilde{V}_{s}\left(V_{s}\right)\right\|_{\infty} \\
& \leq \sup _{\mathcal{C}_{\frac{1}{10} \lambda_{s} \eta_{s}}\left(V_{s}\right)}\left\|\frac{\partial \widetilde{V}_{s}}{\partial V}\right\|_{l^{\infty} \rightarrow l^{\infty}}\left\|X-V_{s}\right\|_{\infty} \\
& \left.<10 \eta_{s}^{-1} \cdot \frac{1}{10} \lambda_{s} \eta_{s} \quad \text { (in view of (5.29)}\right) \\
& =\lambda_{s},
\end{aligned}
$$

that is

$$
\widetilde{V}_{s}\left(\mathcal{C}_{\frac{1}{10} \lambda_{s} \eta_{s}}\left(V_{s}\right)\right) \subseteq \mathcal{C}_{\lambda_{s}}(\omega)
$$


Recalling the estimates in section 4, we have

$$
\begin{aligned}
\left|B_{a 00}^{(m)}\right| & \leq|| R_{1, s+1}||_{\rho_{s+1}}^{+} e^{2 \rho_{s+1}\left(\sum_{n} a_{n}|n|^{\theta}+|m|^{\theta}-\left(n_{1}^{*}\right)^{\theta}\right)} \\
& <\epsilon_{0}^{0.6\left(\frac{3}{2}\right)^{s}} e^{2 \rho_{s+1}\left(\sum_{n} a_{n}|n|^{\theta}+|m|^{\theta}-\left(n_{1}^{*}\right)^{\theta}\right)} .
\end{aligned}
$$

Assuming further

$$
I_{n}(0) \leq e^{-2 r|n|^{\theta}}
$$

and

$$
\rho_{s}<\frac{1}{2} r, \forall s
$$

we obtain

$$
\begin{aligned}
\left|\sum_{a} B_{a 00}^{(m)} \mathcal{M}_{a 00}\right| & \leq \epsilon_{0}^{0.6\left(\frac{3}{2}\right)^{s}} \sum_{a} e^{2 \rho_{s+1}\left(\sum_{n} a_{n}|n|^{\theta}+|m|^{\theta}-\left(n_{1}^{*}\right)^{\theta}\right)} \prod_{n} I_{n}(0)^{a_{n}} \\
& \leq \epsilon_{0}^{0.6\left(\frac{3}{2}\right)^{s}} \sum_{a} e^{2 \rho_{s+1}\left(\sum_{n} a_{n}|n|^{\theta}\right)} \prod_{n} I_{n}(0)^{a_{n}} \\
& \left.\leq \epsilon_{0}^{0.6\left(\frac{3}{2}\right)^{s}} \sum_{a} e^{\sum_{n} 2 \rho_{s+1} a_{n}|n|^{\theta}-\sum_{n} 2 r a_{n}|n|^{\theta}} \text { (in view of (5.31) }\right) \\
& \left.\leq \epsilon_{0}^{0.6\left(\frac{3}{2}\right)^{s}} \sum_{a} e^{-r\left(\sum_{n} a_{n}|n|^{\theta}\right)} \text { (in view of (15.32)}\right) \\
& \leq \epsilon_{0}^{0.6\left(\frac{3}{2}\right)^{s}} \prod_{n}\left(1-e^{-r n^{\theta}}\right)^{-1} \text { (by Lemma 7.2) } \\
& \leq\left(\frac{1}{r}\right)^{C(\theta) r^{-\frac{1}{\theta}}} \epsilon_{0}^{0.6\left(\frac{3}{2}\right)^{s}} \text { (by Lemma 7.4). }
\end{aligned}
$$

By (5.33), we have

$$
\begin{aligned}
\left|\widetilde{V}_{m, s+1}-\widetilde{V}_{m, s}\right| & <\left(\frac{1}{r}\right)^{C(\theta) r^{-\frac{1}{\theta}}} \epsilon_{0}^{0.6\left(\frac{3}{2}\right)^{s}} \\
& <\epsilon_{s}^{0.5} \text { (for } \epsilon_{0} \text { small enough) }
\end{aligned}
$$

which verifies (5.9). Further applying Cauchy's estimate on $\mathcal{C}_{\lambda_{s} \eta_{s}}\left(V_{s}\right)$, one gets

$$
\begin{aligned}
\sum_{n \in \mathbb{Z}}\left|\frac{\partial \widetilde{V}_{m, s+1}}{\partial V_{n}}-\frac{\partial \widetilde{V}_{m, s}}{\partial V_{n}}\right| & \leq C(\theta) \frac{\left\|\widetilde{V}_{s+1}-\widetilde{V}_{s}\right\|_{\infty}}{\lambda_{s} \eta_{s}} \\
& \leq C(\theta) \frac{\epsilon_{s}^{0.5}}{\lambda_{s} \eta_{s}} \\
& \leq e^{C(\theta)\left(\ln \frac{1}{\epsilon_{s+1}}\right)^{\frac{4}{4+\theta}}-\frac{1}{3} \ln \frac{1}{\epsilon_{s+1}}}\left(\frac{1}{\eta_{s}}\right) \\
& \leq e^{-\frac{1}{4} \ln \frac{1}{\epsilon_{s+1}}}\left(\frac{1}{\eta_{s}}\right) \quad \text { (for } \epsilon_{0} \text { small enough) } \\
& =\frac{1}{\eta_{s}} \epsilon_{0}^{\frac{1}{4}\left(\frac{3}{2}\right)^{s+1}} .
\end{aligned}
$$

Since

$$
\eta_{s+1}=\frac{1}{20} \lambda_{s} \eta_{s}
$$


THE STABILITY OF FULL DIMENSIONAL KAM TORI FOR NONLINEAR SCHRÖDINGER EQUATIO\&Y

it follows that

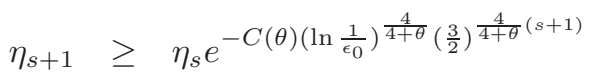

$$
\begin{aligned}
& \geq \eta_{s} e^{-C(\theta) \ln \frac{1}{\epsilon_{0}} \cdot\left(\frac{3}{2}\right)^{\frac{5}{5+\theta} s}} \quad \text { (for } \epsilon_{0} \text { small enough) } \\
& =\eta_{s} \epsilon_{0}^{C(\theta)\left(\frac{3}{2}\right)^{\frac{5 s}{5+\theta}}},
\end{aligned}
$$

and hence by iterating (5.36) implies

$$
\begin{aligned}
\eta_{s} & \geq \eta_{0} \epsilon_{0}^{C(\theta) \sum_{i=0}^{s-1}\left(\frac{3}{2}\right)^{\frac{5 i}{\theta+5}}} \\
& =\eta_{0} \epsilon_{0}(\theta) \frac{\left(\frac{3}{2}\right)^{\frac{5 s}{\theta+5}}-1}{\left(\frac{3}{2}\right)^{\frac{5}{\theta+5}}-1} \\
& >\epsilon_{0}^{C(\theta)\left(\frac{3}{2}\right)^{\frac{5 s}{\theta+5}}} \\
& \geq \epsilon_{0}^{\frac{1}{100}\left(\frac{3}{2}\right)^{s}} \quad \text { (for } \epsilon_{0} \text { small enough). }
\end{aligned}
$$

On $\mathcal{C}_{\frac{1}{10} \lambda_{s} \eta_{s}}\left(V_{s}\right)$, for any $m$, we deduce from (5.35), (5.37) and the assumption (5.3) that

$$
\begin{aligned}
\sum_{n \in \mathbb{Z}}\left|\frac{\partial \widetilde{V}_{m, s+1}}{\partial V_{n}}-\delta_{m n}\right| & \leq \sum_{n \in \mathbb{Z}}\left|\frac{\partial \widetilde{V}_{m, s+1}}{\partial V_{n}}-\frac{\partial \widetilde{V}_{m, s}}{\partial V_{n}}\right|+\sum_{n \in \mathbb{Z}}\left|\frac{\partial \widetilde{V}_{m, s}}{\partial V_{n}}-\delta_{m n}\right| \\
& \leq \epsilon_{0}^{\left(\frac{3}{8}-\frac{1}{100}\right)\left(\frac{3}{2}\right)^{s}}+d_{s} \epsilon_{0}^{\frac{1}{10}} \\
& <d_{s+1} \epsilon_{0}^{\frac{1}{10}}
\end{aligned}
$$

and consequently

$$
\left\|\frac{\partial \widetilde{V}_{s+1}}{\partial V}-I\right\|_{l^{\infty} \rightarrow l^{\infty}}<d_{s+1} \epsilon_{0}^{\frac{1}{10}},
$$

which verifies (5.3) for $s+1$.

Finally, we will freeze $\omega$ by invoking an inverse function theorem. Consider the following functional equation

$$
\widetilde{V}_{s+1}\left(V_{s+1}\right)=\omega, V_{s+1} \in \mathcal{C}_{\frac{1}{10} \lambda_{s} \eta_{s}}\left(V_{s}\right)
$$

from (5.38) and the standard inverse function theorem implies (5.39) having a solution $V_{s+1}$, which verifies (5.2) for $s+1$. Rewriting (5.39) as

$$
V_{s+1}-V_{s}=\left(I-\widetilde{V}_{s+1}\right)\left(V_{s+1}\right)-\left(I-\widetilde{V}_{s+1}\right)\left(V_{s}\right)+\left(\widetilde{V}_{s}-\widetilde{V}_{s+1}\right)\left(V_{s}\right),
$$

and by using (5.34) (5.38) implies

$$
\left\|V_{s+1}-V_{s}\right\|_{\infty} \leq\left(1+d_{s+1}\right) \epsilon_{0}^{\frac{1}{10}}\left\|V_{s+1}-V_{s}\right\|_{\infty}+\epsilon_{s}^{0.5}<2 \epsilon_{s}^{0.5} \ll \lambda_{s} \eta_{s}
$$

which verifies (5.10) and completes the proof of the iterative lemma.

We are now in a position to prove the convergence. To apply iterative lemma with $s=0$, set

$$
V_{0}=\omega, \quad \widetilde{V}_{0}=i d, \quad \eta_{0}=1-\sup _{n \in \mathbb{Z}}\left|\omega_{n}\right|, \quad \rho_{0}=\frac{r}{20}, \quad \epsilon_{0}=C \epsilon,
$$


and consequently (5.2)-(5.6) with $s=0$ are satisfied. Hence, the iterative lemma applies, and we obtain a decreasing sequence of domains $D_{s} \times \mathcal{C}_{\eta_{s}}\left(V_{s}\right)$ and a sequence of transformations

$$
\Phi^{s}=\Phi_{1} \circ \cdots \circ \Phi_{s}: \quad D_{s} \times \mathcal{C}_{\eta_{s}}\left(V_{s}\right) \rightarrow D_{0} \times \mathcal{C}_{\eta_{0}}\left(V_{0}\right)
$$

such that $H \circ \Phi^{s}=N_{s}+P_{s}$ for $s \geq 1$. Moreover, the estimates (5.7)-(5.10) hold. Thus we can show $V_{s}$ converge to a limit $V_{*}$ with the estimate

$$
\left\|V_{*}-\omega\right\|_{\infty} \leq \sum_{s=0}^{\infty} 2 \epsilon_{s}^{0.5}<\epsilon_{0}^{0.4}
$$

and $\Phi^{s}$ converge uniformly on $D_{*} \times\left\{V_{*}\right\}$, where $D_{*}=\left\{\left(q_{n}\right)_{n \in \mathbb{Z}}: \frac{2}{3} \leq\left|q_{n}\right| e^{r|n|^{\theta}} \leq\right.$ $\left.\frac{5}{6}\right\}$, to $\Phi: D_{*} \times\left\{V_{*}\right\} \rightarrow D_{0}$ with the estimates

$$
\begin{aligned}
& \|\Phi-i d\|_{r, \infty} \leq \epsilon_{s}^{0.4} \\
& \|D \Phi-I\|_{(r, \infty) \rightarrow(r, \infty)} \leq \epsilon_{s}^{0.4} .
\end{aligned}
$$

Hence

$$
H_{*}=H \circ \Phi=N_{*}+R_{2, *},
$$

where

$$
N_{*}=\sum_{n \in \mathbb{Z}}\left(n^{2}+\omega_{n}\right)\left|q_{n}\right|^{2}
$$

and

$$
\left\|R_{2, *}\right\|_{\frac{r}{10}}^{+} \leq \frac{7}{6} \epsilon_{0}
$$

By (5.21), the Hamiltonian vector field $X_{R_{2, *}}$ is a bounded map from $\mathfrak{H}_{r, \infty}$ into $\mathfrak{H}_{r, \infty}$. Taking

$$
I_{n}(0)=\frac{3}{4} e^{-2 r|n|^{\theta}}
$$

we get an invariant torus $\mathcal{T}$ with frequency $\left(n^{2}+\omega_{n}\right)_{n \in \mathbb{Z}}$ for $X_{H_{*}}$. Finally, by $X_{H} \circ \Phi=D \Phi \cdot X_{H_{*}}, \Phi(\mathcal{T})$ is the desired invariant torus for the NLS (1.3). Moreover, we deduce the torus $\Phi(\mathcal{T})$ is linearly stable from the fact that (5.42) is a normal form of order 2 around the invariant torus.

\section{Long time stability of FUll Dimensional KAM TORI}

In this section, we would like to study the long time stability of the invariant torus $\Phi(\mathcal{T})$. To this end, we will construct a normal form of order $M$ in the neighborhood of $\mathcal{T}=\left(I_{n}(0)=\frac{3}{4} e^{-2 r|n|^{\theta}}\right)_{n \in \mathbb{Z}}$ firstly (see (5.45)). Precisely, given a $\tau \ll \epsilon$, define the neighborhood of the torus $\mathcal{T}$ by

$$
\widetilde{D}(\tau)=\left\{q:\|J\|_{r, \infty}<\tau, \text { where } J=I-I(0)\right\}
$$

and one has the following theorem:

Theorem 6.1. Consider the normal form of order 2 (see 5.42)

$$
H_{*}=H \circ \Phi=N_{*}+R_{2, *} .
$$

If $\omega=\left(\omega_{n}\right)_{n \in \mathbb{Z}}$ is Diophantine, then there is a symplectic map

$$
\Psi: \widetilde{D}(\tau) \rightarrow \widetilde{D}(2 \tau)
$$


THE STABILITY OF FULL DIMENSIONAL KAM TORI FOR NONLINEAR SCHRÖDINGER EQUATION

such that

$$
H_{*} \circ \Psi=N_{*}+Z+Q
$$

where $Z$ is the integrable term depending on the variables $I$ and the remainder term $Q$ satisfies

$$
\sup _{\widetilde{D}(\tau)}\left\|X_{Q_{(M+1)}}\right\|_{r, \infty} \leq \tau^{\frac{1}{4}|\ln \tau|^{\frac{\theta}{10}}} .
$$

Firstly, we will construct a normal form of order 3 around the tori $\mathcal{T}$ based on a standard normal form procedure where noting $H_{*}$ is already a normal form of order 2. Firstly, rewrite $R_{2, *}$ as

$$
R_{2, *}=\sum_{j \geq 2} R_{j}
$$

with

$$
R_{j}=\sum_{|l|=j} J^{l} \sum_{a, k, k^{\prime}} R_{2, * ; a k k^{\prime}}^{(l)} \mathcal{M}_{a k k^{\prime}}
$$

Then we have the following lemma

Lemma 6.2. Consider the normal form of order 2 (see (5.42))

$$
H_{*}=H \circ \Phi=N_{*}+R_{2, *} .
$$

If $\omega=\left(\omega_{n}\right)_{n \in \mathbb{Z}}$ is Diophantine, then there exists a symplectic map $\Psi_{2}=\left.X_{F_{2}}^{t}\right|_{t=1}$ such that

$$
H_{3}:=H_{*} \circ \Psi_{2}=N_{*}+Z_{3}+Q_{3},
$$

where

$$
\begin{gathered}
Z_{3}=\sum_{|l|=2} J^{l} \sum_{\substack{a, k, k^{\prime} \\
k=k^{\prime}}} R_{2, * ; a k k^{\prime}}^{(l)} \mathcal{M}_{a k k^{\prime}} \\
Q_{3}=\sum_{j \geq 3} Q_{3 j}
\end{gathered}
$$

with

$$
Q_{3 j}=\sum_{|l|=j} J^{l} \sum_{a, k, k^{\prime}} Q_{3 ; a k k^{\prime}}^{(l)} \mathcal{M}_{a k k^{\prime}}
$$

Moreover, the following estimates hold:

and

$$
\left\|F_{2}\right\|_{\frac{r}{10}+\frac{r}{120}} \leq C_{1}(r, \theta, \gamma) \cdot\left\|R_{2, *}\right\|_{\frac{r}{10}+\frac{r}{240}},
$$

where $C_{1}(r, \theta, \gamma)$ and $C_{2}(r, \theta, \gamma)$ are positive constants depending on $r, \theta, \gamma$ only.

\section{Proof. Step 1. The derivative of the homological equation}

Let

$$
F_{2}=\sum_{|l|=2} J^{l} \sum_{a, k, k^{\prime}} F_{2 ; a k k^{\prime}}^{(l)} \mathcal{M}_{a k k^{\prime}}
$$

and let $\Psi_{2}=\left.X_{F_{2}}^{t}\right|_{t=1}$ be the time-1 map of the Hamiltonian vector field $X_{F_{2}}$. 
Using Taylor's formula,

$$
\begin{aligned}
H_{3}:= & \left.H_{*} \circ X_{F_{2}}^{t}\right|_{t=1} \\
= & \left(N_{*}+\left.R_{2, *} \circ X_{F_{2}}^{t}\right|_{t=1}\right. \\
= & N_{*}+\left\{N_{*}, F_{2}\right\}+\sum_{n \geq 2} \frac{1}{n !} \underbrace{\left\{\cdots\left\{N_{*}, F_{2}\right\}, F_{2}, \cdots, F_{2}\right\}}_{n-\text { fold }} \\
& +R_{2}+\sum_{n \geq 1} \frac{1}{n !} \underbrace{\left\{\cdots\left\{R_{2}, F_{2}\right\}, F_{2}, \cdots, F_{2}\right\}}_{n-\text { fold }} \\
& +\sum_{n \geq 0} \frac{1}{n !} \underbrace{\left.\left\{\cdots \sum_{j \geq 3} R_{j}, F_{2}\right\}, F_{2}, \cdots, F_{2}\right\}}_{n-\text { fold }} .
\end{aligned}
$$

Then we obtain the homological equation

$$
\left\{N_{*}, F_{2}\right\}+R_{2}=Z_{3},
$$

where $Z_{3}$ is given by (6.5).

If the homological equation (6.10) is solvable, then we define

$$
\begin{aligned}
Q_{3}= & \sum_{n \geq 2} \frac{1}{n !} \underbrace{\left\{\cdots\left\{N_{*}, F_{2}\right\}, F_{2}, \cdots, F_{2}\right\}}_{n-\text { fold }} \\
& +\sum_{n \geq 1} \frac{1}{n !} \underbrace{\left\{\cdots\left\{R_{2}, F_{2}\right\}, F_{2}, \cdots, F_{2}\right\}}_{n-\text { fold }} \\
& +\sum_{n \geq 0} \frac{1}{n !} \underbrace{\left\{\cdots\left\{\sum_{j \geq 3} R_{j}, F_{2}\right\}, F_{2}, \cdots, F_{2}\right\}}_{n-\text { fold }}
\end{aligned}
$$

and one has

$$
H_{3}=N_{*}+Z_{3}+Q_{3}
$$

Step 2. The solution of the homological equation (6.10). It is easy to show that the solution of the homological equation is given by

$$
F_{2 ; a k k^{\prime}}^{(l)}=\frac{R_{2, * ; a k k^{\prime}}^{(l)}}{\sum_{n}\left(k_{n}-k_{n}^{\prime}\right)\left(n^{2}+\omega_{n}\right)} .
$$

Moreover, the inequality (6.8) holds in view of the fact that $\omega$ is Diophantine and following the proof of Lemma 3.2 .

Step 3. Estimate the remainder terms $Q_{3}$.

To this end, we will estimate the norm of (6.11)-(6.12) respectively. Without loss of generality, we only consider the following term

$$
\frac{1}{n !} \underbrace{\left\{\cdots\left\{R_{2}, F_{2}\right\}, F_{2}, \cdots, F_{2}\right\}}_{n-\text { fold }},
$$


THE STABILITY OF FULL DIMENSIONAL KAM TORI FOR NONLINEAR SCHRÖDINGER EQUATIOA

which is in (6.11). By a direct calculation, one has

$$
\begin{aligned}
& \|\frac{1}{n !} \underbrace{\left\{\cdots\left\{R_{2}, F_{2}\right\}, F_{2}, \cdots, F_{2}\right\}}_{n \text {-fold }}\|_{\frac{r}{10}+\frac{r}{80}} \leq \frac{1}{n !}\left(C(r, \theta)\left\|F_{2}\right\|_{\frac{r}{10}}+\frac{r}{120}\right)^{n}\left(\frac{n}{r}\right)^{n}\left\|R_{2}\right\|_{\frac{r}{10}+\frac{r}{240}}
\end{aligned}
$$

(following the proof of (4.22) and $C(r, \theta)$ is a positive constant depending on $r$ and $\theta$ only)

$$
\leq \frac{1}{n !}\left(C(r, \theta) \cdot C_{1}(r, \theta, \gamma)\right)^{n}\left(\frac{n}{r}\right)^{n}\left\|R_{2}\right\|_{\frac{r}{10}+\frac{r}{240}}^{n+1}
$$

(in view of the first inequality in (6.8)

$$
\begin{aligned}
& \left.\leq\left(\frac{e}{r} \cdot C(r, \theta) \cdot C_{1}(r, \theta, \gamma)\right)^{n}\left\|R_{2}\right\|_{\frac{r}{10}+\frac{r}{240}}^{n+1} \quad \text { (in view of } n^{n} / n ! \leq e^{n}\right) \\
& \leq\left(C_{2}(r, \theta, \gamma)\right)^{n}\left\|R_{2, *}\right\|_{\frac{r}{10}+\frac{r}{240}}^{n+1},
\end{aligned}
$$

where

$$
C_{2}(r, \theta, \gamma)=\frac{e}{r} \cdot C(r, \theta) \cdot C_{1}(r, \theta, \gamma)
$$

depends on $r, \theta, \gamma$ and noting

$$
\left\|R_{2}\right\|_{\frac{r}{10}+\frac{r}{240}} \leq\left\|R_{2, *}\right\|_{\frac{r}{10}}+\frac{r}{240}
$$

Finally, note that the term (6.14) contains at least $(2+n) J^{\prime} s$ and we finish the proof of (6.9).

Remark 6.3. In view of (3.17), (5.44) and (6.8), one has

$$
\left\|F_{2}\right\|_{\frac{r}{10}+\frac{r}{120}} \leq \widetilde{C}(r, \theta, \gamma) \epsilon_{0},
$$

where $\widetilde{C}(r, \theta, \gamma)$ is a positive constant depending on $r, \theta, \gamma$ only. For convenience, we choose $\epsilon_{0}$ small enough at the beginning such that

$$
\widetilde{C}(r, \theta, \gamma) \epsilon_{0} \leq 1
$$

and one has

$$
2 C(\rho, \theta)_{\rho=\frac{r}{10}+\frac{r}{120}}\left\|F_{2}\right\|_{\frac{r}{10}+\frac{r}{120}} \leq 1,
$$

where the positive constant $C(\rho, \theta)$ is given in (5.21). Similarly, one has

$$
\left\|Q_{3 j}\right\|_{\frac{r}{10}+\frac{r}{80}} \leq 1
$$

where by choosing $\epsilon_{0}$ smaller depending on $r, \theta, \gamma$ only. Furthermore, one has

$$
\left\|Q_{3}\right\|_{\frac{r}{10}+\frac{r}{80}} \leq \sup _{j}\left\|Q_{3 j}\right\|_{\frac{r}{10}+\frac{r}{80}} \leq 1 .
$$

Given a large $M>0$, now we will construct a normal form of order $M$ around the torus $\mathcal{T}$. To this end, we give an iterative lemma first. Take

$$
\delta=\frac{r}{80 M} .
$$

For $s \geq 3$, denote

$$
\rho_{s}=\frac{r}{10}+\frac{r}{80}+2(s-3) \delta .
$$


In view of the following two constants

$$
C_{1}(\delta, \theta, \gamma)=\frac{e^{3}}{\gamma} \cdot e^{C(\theta) \delta^{-\frac{5}{\theta}}}
$$

and

$$
C_{2}(\delta, \theta)=\frac{1}{\delta}\left(\frac{1}{\delta}\right)^{C(\theta) \delta^{-\frac{1}{\theta}}},
$$

which are given in Lemma 3.2 (see (3.18)) and Lemma 4.1 (see (4.2) ) respectively, define

$$
C(\delta, \theta, \gamma)=\frac{e}{\delta} \cdot C_{1}(\delta, \theta, \gamma) \cdot C_{2}(\delta, \theta)
$$

Lemma 6.4. Consider the normal form of order $s(s \geq 3)$

$$
H_{s}=N_{*}+Z_{s}+Q_{s}
$$

where

$$
\begin{gathered}
Z_{s}=\sum_{2 \leq j \leq s-1} Z_{s j}, \\
Q_{s}=\sum_{j \geq s} Q_{s j}
\end{gathered}
$$

with

$$
Z_{s j}=\sum_{|l|=j} J^{l} \sum_{\substack{a, k, k^{\prime} \\ k=k^{\prime}}} Z_{s ; a k k^{\prime}}^{(l)} \mathcal{M}_{a k k^{\prime}}
$$

and

$$
Q_{s j}=\sum_{|l|=j} J^{l} \sum_{a, k, k^{\prime}} Q_{s ; a k k^{\prime}}^{(l)} \mathcal{M}_{a k k^{\prime}}
$$

When $s \geq 3$, suppose $Z_{s j}$ and $Q_{s j}$ satisfy the following estimates

$$
\left\|Z_{s j}\right\|_{\rho_{s}} \leq(C(\delta, \theta, \gamma))^{(s-3) j}
$$

and

$$
\left\|Q_{s j}\right\|_{\rho_{s}} \leq(C(\delta, \theta, \gamma))^{(s-3) j} .
$$

Then there exists a symplectic map $\Psi_{s}=\left.X_{F_{s}}^{t}\right|_{t=1}$ such that

$$
H_{s+1}:=H_{s} \circ \Psi_{s}=N_{*}+Z_{s+1}+Q_{s+1},
$$

where

$$
\begin{aligned}
Z_{s+1} & =\sum_{2 \leq j \leq s} Z_{(s+1) j}, \\
Q_{s+1} & =\sum_{j \geq s+1} Q_{(s+1) j},
\end{aligned}
$$

with

$$
Z_{(s+1) j}=\sum_{|l|=j} J^{l} \sum_{\substack{a, k, k^{\prime} \\ k=k^{\prime}}} Z_{s+1 ; a k k^{\prime}}^{(l)} \mathcal{M}_{a k k^{\prime}}
$$


THE STABILITY OF FULL DIMENSIONAL KAM TORI FOR NONLINEAR SCHRÖDINGER EQUATION

and

$$
Q_{(s+1) j}=\sum_{|l|=j} J^{l} \sum_{a, k, k^{\prime}} Q_{s+1 ; a k k^{\prime}}^{(l)} \mathcal{M}_{a k k^{\prime}}
$$

Moreover, the following estimates hold:

$$
\begin{gathered}
\left\|F_{s}\right\|_{\rho_{s+1}} \leq C_{1}(\delta, \theta, \gamma) \cdot(C(\delta, \theta, \gamma))^{(s-3) s}, \\
\left\|Z_{(s+1) j}\right\|_{\rho_{s+1}} \leq(C(\delta, \theta, \gamma))^{(s-2) j},
\end{gathered}
$$

and

$$
\left\|Q_{(s+1) j}\right\|_{\rho_{s+1}} \leq(C(\delta, \theta, \gamma))^{(s-2) j} .
$$

Proof. The details of the proof will be given in Appendix.

\section{Proof of Theorem 6.1.}

Proof. In view of Lemma 6.2 and Lemma 6.4, we define

$$
\Psi:=\Psi_{2} \circ \cdots \circ \Psi_{M} .
$$

Then one has

$$
H_{*} \circ \Psi=N_{*}+Z_{M+1}+Q_{M+1},
$$

and $Z_{M+1}$ and $Q_{M+1}$ satisfies the estimates (6.34) and (6.35) with $s=M$ respectively.

For fixed $0<\tau \ll 1$, we choose

$$
M=\frac{1}{3}|\ln \tau|^{\theta / 10} .
$$

Now we will estimate the norm of the symplectic map $\Psi$ and the remainder term $Q_{M+1}$ respectively.

In view of (6.16), (i.e. $\left.\delta=\frac{r}{80 M}\right),(6.18)$ and (6.19), one has

$$
C_{1}(\delta, \theta, \gamma)=\frac{e^{3}}{\gamma} \cdot e^{C(\theta)\left(\frac{r}{80 M}\right)^{-\frac{5}{\theta}}} \leq e^{M^{\frac{6}{\theta}}}
$$

and

$$
C_{2}(\delta, \theta)=\frac{80 M}{r} \cdot\left(\frac{80 M}{r}\right)^{C(\theta)\left(\frac{r}{80 M}\right)^{-\frac{1}{\theta}}} \leq M^{M^{\frac{2}{\theta}}},
$$

where letting $M$ large enough depending on $\gamma, r, \theta$.

For $s \geq 3$ in view of (6.33), one has

$$
\left\|F_{s}\right\|_{\rho_{s+1}} \leq e^{M^{\frac{6}{\theta}}} \cdot\left(M^{M^{\frac{2}{\theta}}}\right)^{(s-3) s} \leq e^{M^{\frac{10}{\theta}}},
$$

Based on (5.21) in Lemma 5.2 and (6.41), one has

$$
\sup _{\widetilde{D}(\tau)}\left\|X_{F_{s}}\right\|_{r, \infty} \leq C(r, \theta) \cdot e^{M^{10 / \theta}} \tau^{s-1},
$$

where noting that $F_{s}$ contains $s J^{\prime} s$. Hence, in view of (6.38) one has

$$
\left\|\Psi_{s}-i d\right\|_{r, \infty} \leq C(r, \theta) \tau^{s-\frac{4}{3}} .
$$

For $s=2$ and in view of (6.15), one has

$$
\left\|\Psi_{2}-i d\right\|_{r, \infty} \leq \frac{1}{2} \tau
$$


Based on (6.43) and (6.44), we have

$$
\|\Psi-i d\|_{r, \infty} \leq \tau
$$

Now we would like to estimate the remainder term $Q_{M+1}$. In view of (6.16) and (6.17), one has

$$
\rho_{M+1} \leq \frac{11 r}{80} .
$$

Moreover, based on (6.35) for $s=M$, we have

$$
\left\|Q_{(M+1) j}\right\|_{\frac{11 r}{80}} \leq(C(\delta, \theta, \gamma))^{(M-2) j} .
$$

Following the proof of (6.42), one has

$$
\sup _{\widetilde{D}(\tau)}\left\|X_{Q_{(M+1) j}}\right\|_{r, \infty} \leq C(r, \theta) \tau^{j-\frac{3}{2}},
$$

where noting that $Q_{(M+1) j}$ contains $j J^{\prime} s$. Furthermore, we finish the proof of (6.3) by noting $M=\frac{1}{3}|\ln \tau|^{\frac{\theta}{10}}$. Until now, we construct a normal form of order $\frac{1}{3}|\ln \tau|^{\frac{\theta}{10}}$ around the torus $\mathcal{T}$. Following the proof of Corollary 2.16 in [6, it is a standard way to obtain the long time stability of the torus $\mathcal{T}$, i.e. we finish the proof of (1.11) in Theorem 1.1.

\section{APPENDix}

Lemma 7.1. Let $\theta \in(0,1)$ and $k_{n}, k_{n}^{\prime} \in \mathbb{N},\left|\widetilde{V}_{n}\right| \leq 2$ for $\forall n \in \mathbb{Z}$. Assume further

$$
\left|\sum_{n \in \mathbb{Z}}\left(k_{n}-k_{n}^{\prime}\right)\left(n^{2}+\widetilde{V}_{n}\right)\right| \leq 1
$$

and

$$
\sum_{n \in \mathbb{Z}}\left(k_{n}-k_{n}^{\prime}\right) n=0
$$

Then one has

$$
\sum_{n \in \mathbb{Z}}\left|k_{n}-k_{n}^{\prime}\right||n|^{\theta / 2} \leq 3 \cdot 6^{\theta / 2} \sum_{i \geq 3}\left|n_{i}\right|^{\theta},
$$

where $\left(n_{i}\right)_{i \geq 1},\left|n_{1}\right| \geq\left|n_{2}\right| \geq\left|n_{3}\right| \geq \cdots$, denote the system $\left\{n: n\right.$ is repeated $k_{n}+k_{n}^{\prime}$ times\}.

Proof. From the definition of $\left(n_{i}\right)_{i \geq 1}$ and (7.2), there exist $\left(\mu_{i}\right)_{i \geq 1}$ with $\mu_{i} \in\{ \pm 1\}$ such that

$$
\sum_{n \in \mathbb{Z}}\left(k_{n}-k_{n}^{\prime}\right) n^{2}=\sum_{i \geq 1} \mu_{i} n_{i}^{2}
$$

and

$$
\sum_{n \in \mathbb{Z}}\left(k_{n}-k_{n}^{\prime}\right) n=\sum_{i \geq 1} \mu_{i} n_{i}=0 .
$$

In view of (7.1), (7.4) and $\left|\widetilde{V}_{n}\right| \leq 2$, one has

$$
\left|\sum_{i \geq 1} \mu_{i} n_{i}^{2}\right| \leq\left|\sum_{n \in \mathbb{Z}}\left(k_{n}-k_{n}^{\prime}\right) \widetilde{V}_{n}\right|+1 \leq 2 \sum_{n \in \mathbb{Z}}\left(k_{n}+k_{n}^{\prime}\right)+1,
$$


THE STABILITY OF FULL DIMENSIONAL KAM TORI FOR NONLINEAR SCHRÖDINGER EQUATIOßף

which implies

$$
\left|n_{1}^{2}+\left(\frac{\mu_{2}}{\mu_{1}}\right) n_{2}^{2}\right| \leq 2 \sum_{i \geq 1} 1+\sum_{i \geq 3} n_{i}^{2}+1 \leq \sum_{i \geq 3}\left(2+n_{i}^{2}\right)+3 .
$$

In the other hand, by (7.5), we obtain

$$
\left|n_{1}+\left(\frac{\mu_{2}}{\mu_{1}}\right) n_{2}\right| \leq \sum_{i \geq 3}\left|n_{i}\right|
$$

To prove the inequality (17.3), we will distinguish two cases:

Case. 1. $\frac{\mu_{2}}{\mu_{1}}=-1$.

Case. 1.1. $n_{1}=n_{2}$.

Then it is to show that

$$
\sum_{n \in \mathbb{Z}}\left|k_{n}-k_{n}^{\prime}\right||n|^{\theta / 2} \leq \sum_{i \geq 3}\left|n_{i}\right|^{\theta / 2} \leq 3 \cdot 6^{\theta / 2} \sum_{i \geq 3}\left|n_{i}\right|^{\theta} .
$$

Case. 1.2. $n_{1} \neq n_{2}$.

Then one has

$$
\begin{aligned}
\left|n_{1}-n_{2}\right|+\left|n_{1}+n_{2}\right| & \leq\left|n_{1}-n_{2}\right|+\left|n_{1}^{2}-n_{2}^{2}\right| \\
& \left.\leq \sum_{i \geq 3}\left|n_{i}\right|+\sum_{i \geq 3}\left(2+n_{i}^{2}\right)+3 \quad \text { (in view of (7.6) and (7.7) }\right) \\
& \leq 6 \sum_{i \geq 3}\left|n_{i}\right|^{2} .
\end{aligned}
$$

Hence

$$
\max \left\{\left|n_{1}\right|,\left|n_{2}\right|\right\} \leq \max \left\{\left|n_{1}-n_{2}\right|,\left|n_{1}+n_{2}\right|\right\} \leq 6 \sum_{i \geq 3}\left|n_{i}\right|^{2},
$$

where the last inequality is based on (7.8). For $j=1,2$, one has

$$
\left|n_{j}\right|^{\theta / 2} \leq 6^{\theta / 2}\left(\sum_{i \geq 3}\left|n_{i}\right|^{2}\right)^{\theta / 2} \leq 6^{\theta / 2} \sum_{i \geq 3}\left|n_{i}\right|^{\theta},
$$

where the last inequality is based on the fact that the function $|x|^{\theta / 2}$ is a concave function for $0<\theta<1$. Therefore,

$$
\left|n_{1}\right|^{\theta / 2}+\left|n_{2}\right|^{\theta / 2} \leq 2 \cdot 6^{\theta / 2} \sum_{i \geq 3}\left|n_{i}\right|^{\theta} .
$$

Now one has

$$
\begin{aligned}
\sum_{n \in \mathbb{Z}}\left|k_{n}-k_{n}^{\prime}\right||n|^{\theta / 2} & \leq \sum_{n \in \mathbb{Z}}\left(k_{n}+k_{n}^{\prime}\right)|n|^{\theta / 2} \\
& =\sum_{i \geq 1}\left|n_{i}\right|^{\theta / 2} \\
& \leq\left(\left|n_{1}\right|^{\theta / 2}+\left|n_{2}\right|^{\theta / 2}\right)+\sum_{i \geq 3}\left|n_{i}\right|^{\theta} \\
& \leq\left(2 \cdot 6^{\theta / 2}+1\right) \sum_{i \geq 3}\left|n_{i}\right|^{\theta} \quad \text { (in view of }(\underline{7.9}) \text { ) } \\
& \leq 3 \cdot 6^{\theta / 2} \sum_{i \geq 3}\left|n_{i}\right|^{\theta} .
\end{aligned}
$$


Case. 2. $\frac{\mu_{2}}{\mu_{1}}=1$.

In view of (7.6), one has

$$
n_{1}^{2}+n_{2}^{2} \leq 5 \sum_{i \geq 3}\left|n_{i}\right|^{2}
$$

which implies

$$
\left|n_{j}\right|^{\theta / 2} \leq 5^{\theta / 2}\left(\sum_{i \geq 3}\left|n_{i}\right|^{2}\right)^{\theta / 2} \leq 5^{\theta / 2} \sum_{i \geq 3}\left|n_{i}\right|^{\theta} \quad(j=1,2) .
$$

Therefore,

$$
\left|n_{1}\right|^{\theta / 2}+\left|n_{2}\right|^{\theta / 2} \leq 2 \cdot 5^{\theta / 2} \sum_{i \geq 3}\left|n_{i}\right|^{\theta} .
$$

Following the proof of (7.10), we have

$$
\sum_{n \in \mathbb{Z}}\left|k_{n}-k_{n}^{\prime}\right||n|^{\theta / 2} \leq 3 \cdot 6^{\theta / 2} \sum_{i \geq 3}\left|n_{i}\right|^{\theta} .
$$

Lemma 7.2. Assuming $\theta, \delta \in(0,1)$, then we have the following inequality

$$
\sum_{a \in \mathbb{N}^{\mathbb{Z}}} e^{-\delta \sum_{n \in \mathbb{Z}} a_{n}|n|^{\theta}} \leq \prod_{n \in \mathbb{Z}} \frac{1}{1-e^{-\delta|n|^{\theta}}},
$$

where $|0|:=1$

Proof. $\sum_{a \in \mathbb{N} \mathbb{Z}} e^{-\delta \sum_{n \in \mathbb{Z}} a_{n}|n|^{\theta}} \leq \prod_{n \in \mathbb{Z}}\left(\sum_{a_{n} \in \mathbb{N}} e^{-\delta a_{n}|n|^{\theta}}\right)=\prod_{n \in \mathbb{Z}} \frac{1}{1-e^{-\delta|n|^{\theta}}}$.

Lemma 7.3. Assuming $\theta, \delta \in(0,1)$, then we have

$$
\sum_{n \geq 1} e^{-\delta n^{\theta}} \leq e^{\frac{(\theta-1)}{\theta}} \cdot \frac{2}{\theta}\left(\frac{2(1-\theta)}{\theta}\right)^{\frac{1}{\theta}-1} \delta^{-\frac{1}{\theta}} .
$$

Proof. Obviously, we have

$$
\sum_{n \geq 1} e^{-\delta n^{\theta}} \leq \int_{0}^{+\infty} e^{-\delta x^{\theta}} \mathrm{d} x=\frac{1}{\theta} \int_{0}^{+\infty} e^{-\delta x} x^{\frac{1}{\theta}-1} \mathrm{~d} x .
$$

Let $f(x)=e^{-\frac{1}{2} \delta x} x^{\frac{1}{\theta}-1}$, and it is easy to prove

$$
\max _{x \geq 0} f(x)=f\left(\frac{2(1-\theta)}{\delta \theta}\right)=e^{\frac{\theta-1}{\theta}}\left(\frac{2(1-\theta)}{\delta \theta}\right)^{\frac{1}{\theta}-1} .
$$

Consequently

$$
\begin{aligned}
\text { (17.14) } & \leq \frac{1}{\theta} \cdot e^{\frac{\theta-1}{\theta}} \cdot\left(\frac{2(1-\theta)}{\delta \theta}\right)^{\frac{1}{\theta}-1} \int_{0}^{+\infty} e^{-\frac{\delta}{2} x} \mathrm{~d} x \\
& \leq e^{\frac{(\theta-1)}{\theta}} \cdot \frac{2}{\theta}\left(\frac{2(1-\theta)}{\theta}\right)^{\frac{1}{\theta}-1} \delta^{-\frac{1}{\theta}}
\end{aligned}
$$

which finishes the proof of (7.13). 
THE STABILITY OF FULL DIMENSIONAL KAM TORI FOR NONLINEAR SCHRÖDINGER EQUATIOA

Lemma 7.4. Assuming $\theta \in(0,1), \delta \in\left(0, \frac{1}{e}\right)$, then we have

$$
\prod_{n \in \mathbb{Z}} \frac{1}{1-e^{-\delta|n|^{\theta}}} \leq\left(\frac{1}{\delta}\right)^{C(\theta) \delta^{-\frac{1}{\theta}}},
$$

where $|0|:=1$ and $C(\theta)$ is a positive constant depending on $\theta$ only.

Proof. We write

$$
\begin{aligned}
\prod_{n \in \mathbb{Z}} \frac{1}{1-e^{-\delta|n|^{\theta}}} & =\left(\frac{1}{1-e^{-\delta}}\right) \cdot \prod_{n \geq 1}\left(\frac{1}{1-e^{-\delta n^{\theta}}}\right)^{2} \\
& =\left(\frac{1}{1-e^{-\delta}}\right) \cdot \prod_{1 \leq n \leq N_{\theta}}\left(\frac{1}{1-e^{-\delta n^{\theta}}}\right)^{2} \prod_{n>N_{\theta}}\left(\frac{1}{1-e^{-\delta n^{\theta}}}\right)^{2},
\end{aligned}
$$

where

$$
N_{\theta}=(\ln (3+2 \sqrt{2}))^{\frac{1}{\theta}} \delta^{-\frac{1}{\theta}}
$$

For $\delta \in\left(0, \frac{1}{e}\right)$, one has

$$
\frac{1}{1-e^{-\delta}} \leq \frac{1}{\delta^{2}}
$$

and then

$$
\begin{aligned}
\left(\frac{1}{1-e^{-\delta}}\right) \cdot \prod_{1 \leq n \leq N_{\theta}}\left(\frac{1}{1-e^{-\delta n^{\theta}}}\right)^{2} & \leq\left(\frac{1}{1-e^{-\delta}}\right) \cdot \prod_{1 \leq n \leq N_{\theta}}\left(\frac{1}{1-e^{-\delta}}\right)^{2} \\
& =\left(\frac{1}{1-e^{-\delta}}\right)^{2 N_{\theta}+1} \\
& \leq\left(\frac{1}{\delta}\right)^{4 N_{\theta}+2} \quad \text { in view of (7.19)) } \\
& \leq\left(\frac{1}{\delta}\right)^{6(\ln (3+2 \sqrt{2}))^{\frac{1}{\theta}} \delta^{-\frac{1}{\theta}}} \quad \text { (in view of }(7.18) \text { ). }
\end{aligned}
$$

When $n>N_{\theta}$, one has

$$
\delta n^{\theta}>\ln (3+2 \sqrt{2})
$$

Note that if $x \in(0,3-2 \sqrt{2})$, we have

$$
\ln \left(\frac{1}{1-x}\right) \leq \sqrt{x}
$$

which implies

$$
\ln \left(\frac{1}{1-e^{-\delta x}}\right) \leq e^{-\frac{1}{2} \delta x} \text { for } \delta x>\ln (3+2 \sqrt{2})
$$


Hence

$$
\begin{aligned}
\prod_{n>N_{\theta}}\left(\frac{1}{1-e^{-\delta n^{\theta}}}\right)^{2} & =e^{\sum_{n>N_{\theta}} 2 \ln \left(\frac{1}{1-e^{-\delta n^{\theta}}}\right)} \\
& \leq e^{\left.\sum_{n>N_{\theta}} 2 e^{-\frac{1}{2} \delta n^{\theta}} \quad \text { (in view of (7.21) and (7.22) }\right)} \\
& \leq e^{C_{1}(\theta) \delta^{-\frac{1}{\theta}}},
\end{aligned}
$$

where the last inequality is based on Lemma 7.3 and

$$
C_{1}(\theta)=2^{\frac{1}{\theta}} e^{\frac{(\theta-1)}{\theta}} \cdot \frac{4}{\theta}\left(\frac{2(1-\theta)}{\theta}\right)^{\frac{1}{\theta}-1} .
$$

Recalling $\delta \in\left(0, \frac{1}{e}\right)$, then the estimate (7.16) follows from (7.17), (7.20) and (7.23), where

$$
C(\theta)=C_{1}(\theta)+6(\ln (3+2 \sqrt{2}))^{\frac{1}{\theta}} .
$$

Lemma 7.5. Assuming $f_{p}(x)=x^{p} e^{-\delta x}(p=1,2)$, then we have

$$
\max _{x \geq 0} f_{p}(x) \leq \frac{1}{\delta^{p}} \text {. }
$$

Proof. Since $f_{p}(x)=x^{p} e^{-\delta x}$, we have

$$
f_{p}^{\prime}(x)=p x^{p-1} e^{-\delta x}-\delta x^{p} e^{-\delta x}=\left(p x^{p-1}-\delta x^{p}\right) e^{-\delta x} .
$$

Hence we get

$$
f_{p}^{\prime}\left(\frac{p}{\delta}\right)=0
$$

and it is easy to see

$$
\max _{x \geq 0} f_{p}(x)=f_{p}\left(\frac{p}{\delta}\right)=\frac{p^{p}}{\delta^{p}} e^{-p} \leq \frac{1}{\delta^{p}} \quad(p=1,2) .
$$

Lemma 7.6. Assuming $\theta, \delta \in(0,1)$ and $a=\left(a_{n}\right)_{n \in \mathbb{Z}} \in \mathbb{N}^{\mathbb{Z}}$, then we have

$$
\prod_{n \in \mathbb{Z}}\left(1+a_{n}^{p}\right) e^{-2 \delta a_{n}|n|^{\theta}} \leq\left(\frac{1}{\delta}\right)^{3 p \delta^{-\frac{1}{\theta}}},
$$

where $p=1,2$ and $|0|:=1$.

Proof. Firstly, we note

$$
\prod_{n \in \mathbb{Z}}\left(1+a_{n}^{p}\right) e^{-2 \delta a_{n}|n|^{\theta}}=\prod_{n \in \mathbb{Z}: a_{n} \geq 1}\left(1+a_{n}^{p}\right) e^{-2 \delta a_{n}|n|^{\theta}}
$$

and we can assume $a_{n} \geq 1$ for $\forall n \in \mathbb{Z}$ in what follows. Thus one has

$$
\begin{aligned}
\left(1+a_{n}^{p}\right) e^{-2 \delta a_{n}|n|^{\theta}} & \leq 2 a_{n}^{p} e^{-2 \delta a_{n}|n|^{\theta}} \\
& \leq \frac{1}{|n|^{p \theta}} \cdot\left(2^{\frac{1}{p}} a_{n}|n|^{\theta}\right)^{p} e^{-\delta 2^{\frac{1}{p}} a_{n}|n|^{\theta}} \\
& \leq \frac{1}{|n|^{p \theta}} \cdot \frac{1}{\delta^{p}}
\end{aligned}
$$


THE STABILITY OF FULL DIMENSIONAL KAM TORI FOR NONLINEAR SCHRÖDINGER EQUATION where the last inequality is based on (7.24). Hence, if $|n| \geq \delta^{-1 / \theta}$, one has

$$
\left(1+a_{n}^{p}\right) e^{-2 \delta a_{n}|n|^{\theta}} \leq 1 .
$$

Therefore,

$$
\begin{aligned}
\prod_{n \in \mathbb{Z}}\left(1+a_{n}^{p}\right) e^{-2 \delta a_{n}|n|^{\theta}} & =\left(\prod_{\substack{1 \leq|n|<\delta^{-1 / \theta} \\
(\text { noting that }|0|:=1)}}\left(1+a_{n}^{p}\right) e^{-2 \delta a_{n}|n|^{\theta}}\right)\left(\prod_{|n| \geq \delta^{-1 / \theta}}\left(1+a_{n}^{p}\right) e^{-2 \delta a_{n}|n|^{\theta}}\right) \\
\leq & \prod_{1 \leq|n|<\delta^{-1 / \theta}}\left(1+a_{n}^{p}\right) e^{-2 \delta a_{n}|n|^{\theta}} \quad \text { (in view of }(\underline{7.28)}) \\
\leq & \left.\prod_{1 \leq|n|<\delta^{-1 / \theta}} \frac{1}{|n|^{p \theta} \delta^{p}} \quad \text { in view of }(\underline{7.27})\right) \\
\leq & \left(\frac{1}{\delta}\right)^{2 p \delta^{-\frac{1}{\theta}}+p} \\
\leq & \left(\frac{1}{\delta}\right)^{3 p \delta^{-\frac{1}{\theta}}} .
\end{aligned}
$$

Lemma 7.7. Let $a, k, k^{\prime} \in \mathbb{N}^{\mathbb{Z}}, \theta \in(0,1)$, and $0<\delta \ll 1$ (depending only on $\theta$ ). Let further $\left(n_{i}\right)_{i \geq 1},\left|n_{1}\right| \geq\left|n_{2}\right| \geq\left|n_{3}\right| \geq \cdots$, denote the system $\{|n|: n$ is repeated $2 a_{n}+k_{n}+k_{n}^{\prime}$ times\}. Then we have

$$
\prod_{|m| \leq\left|n_{1}\right|}\left(1+l_{m}^{2}\right) e^{-\delta \sum_{|m| \leq\left|n_{3}\right|} l_{m}|m|^{\theta}} \leq\left(\frac{1}{\delta}\right)^{C(\theta) \delta^{-\frac{1}{\theta}}},
$$

where

$$
l_{n}=\#\left\{j: n=n_{j}\right\},
$$

and $C(\theta)$ is a positive constant depending only on $\theta$.

Proof. To prove (7.29), we distinguish three cases:

Case 1. $\left|n_{1}\right|=\left|n_{2}\right|=\left|n_{3}\right|$. In this case, we have

$$
\begin{aligned}
\prod_{|m| \leq\left|n_{1}\right|}\left(1+l_{m}^{2}\right) e^{-\delta \sum_{|m| \leq\left|n_{3}\right|} l_{m}|m|^{\theta}} & =\prod_{|m| \leq\left|n_{1}\right|}\left(1+l_{m}^{2}\right) e^{-\delta \sum_{i \geq 3}\left|n_{i}\right|^{\theta}} \\
& \leq \prod_{|m| \leq\left|n_{1}\right|}\left(1+l_{m}^{2}\right) e^{-\frac{1}{3} \delta \sum_{i \geq 1}\left|n_{i}\right|^{\theta}} \\
& =\prod_{|m| \leq\left|n_{1}\right|}\left(\left(1+l_{m}^{2}\right) e^{-\frac{1}{3} \delta l_{m}|m|^{\theta}}\right) \\
& \left.\leq\left(\frac{6}{\delta}\right)^{6\left(\frac{6}{\delta}\right)^{-\frac{1}{\theta}}} \quad \text { (in view of }(\underline{7.26})\right) \\
& \leq\left(\frac{1}{\delta}\right)^{C_{1}(\theta) \delta^{-\frac{1}{\theta}}},
\end{aligned}
$$


where the last inequality relies on $0<\delta \ll 1$ and $C_{1}(\theta)$ is a positive constant depending on $\theta$ only.

Case 2. $\left|n_{1}\right|>\left|n_{2}\right|=\left|n_{3}\right|$. In this case, $l_{n_{1}}=1$. Hence, we have

$$
\begin{aligned}
\prod_{|m| \leq\left|n_{1}\right|}\left(1+l_{m}^{2}\right) e^{-\delta \sum_{|m| \leq\left|n_{3}\right|} l_{m}|m|^{\theta}} & =2 \cdot \prod_{|m| \leq\left|n_{2}\right|}\left(1+l_{m}^{2}\right) e^{-\delta \sum_{i \geq 3}\left|n_{i}\right|^{\theta}} \\
& \leq 2 \cdot \prod_{|m| \leq\left|n_{2}\right|}\left(1+l_{m}^{2}\right) e^{-\frac{1}{2} \delta \sum_{i \geq 2}\left|n_{i}\right|^{\theta}} \\
& =2 \cdot \prod_{|m| \leq\left|n_{2}\right|}\left(\left(1+l_{m}^{2}\right) e^{-\frac{1}{2} \delta l_{m}|m|^{\theta}}\right) \\
& \leq\left(\frac{1}{\delta}\right)^{C_{2}(\theta) \delta^{-\frac{1}{\theta}}},
\end{aligned}
$$

where the last inequality follows form the proof of (7.30) and $C_{2}(\theta)$ is a positive constant depending on $\theta$ only.

Case 3. $\left|n_{1}\right| \geq\left|n_{2}\right|>\left|n_{3}\right|$. In this case, $l_{m} \leq 2$ for $m \in\left\{n_{1}, n_{2}\right\}$. Thus, we have

$$
\begin{aligned}
\prod_{|m| \leq\left|n_{1}\right|}\left(1+l_{m}^{2}\right) e^{-\delta \sum_{|m| \leq\left|n_{3}\right|} l_{m}|m|^{\theta}} & =\prod_{m \in\left\{n_{1}, n_{2}\right\}}\left(1+l_{m}^{2}\right) \prod_{|m| \leq\left|n_{3}\right|}\left(\left(1+l_{m}^{2}\right) e^{-\delta l_{m}|m|^{\theta}}\right) \\
& \leq 5 \cdot \prod_{|m| \leq\left|n_{3}\right|}\left(\left(1+l_{m}^{2}\right) e^{-\delta l_{m}|m|^{\theta}}\right) \\
(7.32) & \leq\left(\frac{1}{\delta}\right)^{C_{3}(\theta) \delta^{-\frac{1}{\theta}}},
\end{aligned}
$$

where the last inequality follows form the proof of (7.30) and $C_{3}(\theta)$ is a positive constant depending on $\theta$ only.

In view of (7.30)-(7.32), we finished the proof of (7.29).

\section{The proof of Lemma 3.1}

Proof. Firstly, we will prove the inequality (3.16). Write $\mathcal{M}_{a k k^{\prime}}$ in the form of

$$
\mathcal{M}_{a k k^{\prime}}=\mathcal{M}_{a b l l^{\prime}}=\prod_{n} I_{n}(0)^{a_{n}} I_{n}^{b_{n}} q_{n}^{l_{n}} \bar{q}_{n}^{l_{n}^{\prime}}
$$

where

$$
b_{n}=k_{n} \wedge k_{n}^{\prime}, \quad l_{n}=k_{n}-b_{n}, \quad l_{n}^{\prime}=k_{n}^{\prime}-b_{n}^{\prime}
$$

and $l_{n} l_{n}^{\prime}=0$ for all $n$.

Express the term

$$
\prod_{n} I_{n}^{b_{n}}=\prod_{n}\left(I_{n}(0)+J_{n}\right)^{b_{n}}
$$

by the monomials of the form

$$
\begin{gathered}
\prod_{n} I_{n}(0)^{b_{n}} \\
\sum_{m, b_{m} \geq 1}\left(I_{m}(0)^{b_{m}-1} J_{m}\right)\left(\sum_{n \neq m} \prod_{n} I_{n}(0)^{b_{n}}\right),
\end{gathered}
$$


THE STABILITY OF FULL DIMENSIONAL KAM TORI FOR NONLINEAR SCHRÖDINGER EQUATIOA

$$
\sum_{\substack{m, b_{m} \geq 2 \\ r \leq b_{m}-2}}\left(\sum_{n<m} \prod_{n} I_{n}(0)^{b_{n}}\right)\left(b_{m}\left(b_{m}-1\right) I_{m}(0)^{r} J_{m}^{2} I_{m}^{b_{m}-r-2}\right)\left(\sum_{n>m} \prod_{n} I_{n}^{b_{n}}\right),
$$

and

$$
\begin{gathered}
\sum_{\substack{m_{1}<m_{2}, b_{m_{1}}, b_{m_{2}} \geq 1 \\
r \leq b_{m_{2}}-1}}\left(\sum_{n<m_{1}} \prod_{n} I_{n}(0)^{b_{n}}\right)\left(b_{m_{1}} I_{m_{1}}(0)^{b_{m_{1}}-1} J_{m_{1}}\right) \\
\times\left(\sum_{m_{1}<n<m_{2}} \prod_{n} I_{n}^{b_{n}}\right)\left(b_{m_{1}} I_{m_{1}}(0)^{r} J_{m_{1}} I_{m_{1}}^{b_{m_{1}}-r-1}\right)\left(\sum_{n>m_{2}} \prod_{n} I_{n}^{b_{n}}\right) .
\end{gathered}
$$

Now we will estimate the bounds for the coefficients respectively. Consider the term $\mathcal{M}_{a k k^{\prime}}=\prod_{n} I_{n}(0)^{a_{n}} q_{n}^{k_{n}} \bar{q}_{n}^{k_{n}^{\prime}}$ with fixed $a, k, k^{\prime}$ satisfying $k_{n} k_{n}^{\prime}=0$ for all $n$. It is easy to see that $\mathcal{M}_{a k k^{\prime}}$ comes from some parts of the terms $\mathcal{M}_{\alpha \kappa \kappa^{\prime}}$ with no assumption for $\kappa$ and $\kappa^{\prime}$. For any given $n$ one has

$$
I_{n}(0)^{a_{n}} q_{n}^{k_{n}} \bar{q}_{n}^{k_{n}^{\prime}}=\sum_{\beta_{n}=k_{n} \wedge k_{n}^{\prime}} I_{n}(0)^{\alpha_{n}+\beta_{n}} q_{n}^{\kappa_{n}-\beta_{n}} \bar{q}_{n}^{\kappa_{n}^{\prime}-\beta_{n}} .
$$

Hence,

$$
\alpha_{n}+\beta_{n}=a_{n},
$$

and

$$
\kappa_{n}-\beta_{n}=k_{n}, \quad \kappa_{n}^{\prime}-\beta_{n}=k_{n}^{\prime} .
$$

Therefore, if $0 \leq \alpha_{n} \leq a_{n}$ is chosen, so $\beta_{n}, k_{n}, k_{n}^{\prime}$ are determined. On the other hand,

$$
\begin{aligned}
\left|B_{\alpha \kappa \kappa^{\prime}}\right| \leq & \|R\|_{\rho} e^{\rho\left(\sum_{n}\left(2 \alpha_{n}+\kappa_{n}+\kappa_{n}^{\prime}\right)|n|^{\theta}-2\left(n_{1}^{*}\right)^{\theta}\right)} \\
& (\text { in view of }(2.12)) \\
= & \|R\|_{\rho} e^{\rho\left(\sum_{n}\left(2 \alpha_{n}+\left(k_{n}+a_{n}-\alpha_{n}\right)+\left(k_{n}^{\prime}+a_{n}-\alpha_{n}\right)\right)|n|^{\theta}-2\left(n_{1}^{*}\right)^{\theta}\right)} \\
& (\text { in view of }(\mathbf{7 . 3 3}) \text { and }(\mathbf{7 . 3 4})) \\
= & \|R\|_{\rho} e^{\rho\left(\sum_{n}\left(2 a_{n}+k_{n}+k_{n}^{\prime}\right)|n|^{\theta}-2\left(n_{1}^{*}\right)^{\theta}\right)} .
\end{aligned}
$$

Hence,

$$
\left|B_{a k k^{\prime}}\right| \leq\|R\|_{\rho} \prod_{n}\left(1+a_{n}\right) e^{\rho\left(\sum_{n}\left(2 a_{n}+k_{n}+k_{n}^{\prime}\right)|n|^{\theta}-2\left(n_{1}^{*}\right)^{\theta}\right)} .
$$

Similarly,

$$
\begin{aligned}
\left|B_{a k k^{\prime}}^{(m)}\right| \leq & \|R\|_{\rho}\left(\prod_{n \neq m}\left(1+a_{n}\right)\right)\left(1+a_{m}\right)^{2} e^{\rho\left(\sum_{n}\left(2 a_{n}+k_{n}+k_{n}^{\prime}\right)|n|^{\theta}+2|m|^{\theta}-2\left(n_{1}^{*}\right)^{\theta}\right),} \\
\left|B_{a k k^{\prime}}^{(m, m)}\right| \leq & \|R\|_{\rho}\left(\prod_{n \neq m}\left(1+a_{n}\right)\right)\left(1+a_{m}\right)^{3} e^{\rho\left(\sum_{n}\left(2 a_{n}+k_{n}+k_{n}^{\prime}\right)|n|^{\theta}+4|m|^{\theta}-2\left(n_{1}^{*}\right)^{\theta}\right),} \\
\left|B_{a k k^{\prime}}^{\left(m_{1}, m_{2}\right)}\right| \leq & || R \|_{\rho}\left(\prod_{n<m_{1}}\left(1+a_{n}\right)\right)\left(1+a_{m_{1}}\right)^{2}\left(\prod_{m_{1}<n<m_{2}}\left(1+a_{n}\right)\right) \\
& \times\left(1+a_{m_{2}}\right)^{2} e^{\rho\left(\sum_{n}\left(2 a_{n}+k_{n}+k_{n}^{\prime}\right)|n|^{\theta}+2\left|m_{1}\right|^{\theta}+2\left|m_{2}\right|^{\theta}-2\left(n_{1}^{*}\right)^{\theta}\right) .}
\end{aligned}
$$


In view of (3.13) and (7.35), we have

$$
\left\|R_{0}\right\|_{\rho+\delta}^{+} \leq\|R\|_{\rho} \prod_{n}\left(1+a_{n}\right) e^{-\delta\left(\sum_{n}\left(2 a_{n}+k_{n}+k_{n}^{\prime}\right)|n|^{\theta}-2\left(n_{1}^{*}\right)^{\theta}\right)} .
$$

Now we will show that

$$
\prod_{n}\left(1+a_{n}\right) e^{-\delta\left(\sum_{n}\left(2 a_{n}+k_{n}+k_{n}^{\prime}\right)|n|^{\theta}-2\left(n_{1}^{*}\right)^{\theta}\right)} \leq\left(\frac{1}{\delta}\right)^{C(\theta) \delta^{-\frac{1}{\theta}}}
$$

where $C(\theta)$ is a positive constant depending only on $\theta$.

Case 1. $n_{1}^{*}=n_{3}^{*}$. Then one has

$$
\begin{aligned}
\text { (7.37) } & =\prod_{n}\left(1+a_{n}\right) e^{-\delta \sum_{i \geq 3}\left|n_{i}\right|^{\theta}} \\
& \leq \prod_{n}\left(1+a_{n}\right) e^{-\frac{\delta}{3} \sum_{i \geq 1}\left|n_{i}\right|^{\theta}} \\
& =\prod_{n}\left(1+a_{n}\right) e^{-\frac{\delta}{3} \sum_{n}\left(2 a_{n}+k_{n}+k_{n}^{\prime}\right)|n|^{\theta}} \\
& \leq \prod_{n}\left(\left(1+a_{n}\right) e^{-\frac{2 \delta}{3} a_{n}|n|^{\theta}}\right) \\
& \left.\leq\left(\frac{1}{\delta}\right)^{C(\theta) \delta^{-\frac{1}{\theta}}} \quad \text { (in view of (17.26) }\right) .
\end{aligned}
$$

Case 2. $n_{1}^{*}>n_{2}^{*}=n_{3}^{*}$. In this case, $a_{n}=1$ for $n=n_{1}$. Then we have

$$
\begin{aligned}
\text { (7.37) } & =\left(1+a_{n_{1}}\right)\left(\prod_{|n| \leq n_{2}^{*}}\left(1+a_{n}\right) e^{-\left(2-2^{\theta}\right) \delta \sum_{i \geq 3}\left(n_{i}^{*}\right)^{\theta}}\right) \\
& \leq 2 \cdot \prod_{|n| \leq n_{2}^{*}}\left(1+a_{n}\right) e^{-\frac{1}{2}\left(2-2^{\theta}\right) \delta \sum_{i \geq 2}\left(n_{i}^{*}\right)^{\theta}} \\
& =2 \cdot \prod_{|n| \leq n_{2}^{*}}\left(1+a_{n}\right) e^{-\frac{1}{2}\left(2-2^{\theta}\right) \delta \sum_{|n| \leq n 2}\left(2 a_{n}+k_{n}+k_{n}^{\prime}\right)|n|^{\theta}} \\
& 2 \cdot \prod_{|n| \leq n_{2}^{*}}\left(\left(1+a_{n}\right) e^{-\left(2-2^{\theta}\right) \delta a_{n}|n|^{\theta}}\right) \\
& \left.\leq\left(\frac{1}{\delta}\right)^{C(\theta) \delta^{-\frac{1}{\theta}}} \quad \text { (in view of (17.26) }\right) .
\end{aligned}
$$


THE STABILITY OF FULL DIMENSIONAL KAM TORI FOR NONLINEAR SCHRÖDINGER EQUATIOAY

Case 3. $n_{2}^{*}>n_{3}^{*}$. In this case, $a_{n} \leq 2$ for $n \in\left\{n_{1}, n_{2}\right\}$. Hence

$$
\begin{aligned}
\text { (7.37) } & \leq\left(\prod_{n \in\left\{n_{1}, n_{2}\right\}}\left(1+a_{n}\right)\right)\left(\prod_{|n| \leq n_{3}^{*}}\left(1+a_{n}\right) e^{-\delta \sum_{i \geq 3}\left(n_{i}^{*}\right)^{\theta}}\right) \\
& \leq 2^{2} \cdot \prod_{|n| \leq n_{3}^{*}}\left(1+a_{n}\right) e^{-\delta \sum_{|n| \leq n_{3}^{*}}\left(2 a_{n}+k_{n}+k_{n}^{\prime}\right)|n|^{\theta}} \\
& \leq 2^{2} \cdot \prod_{|n| \leq n_{3}^{*}}\left(\left(1+a_{n}\right) e^{-2 \delta a_{n}|n|^{\theta}}\right) \\
& \left.\leq\left(\frac{1}{\delta}\right)^{C(\theta) \delta^{-\frac{1}{\theta}}} \quad \text { (in view of } \underline{(7.26)}\right) .
\end{aligned}
$$

We finished the proof of (7.37).

Similarly, one has

$$
\left\|R_{1}\right\|_{\rho+\delta}^{+},\left\|R_{2}\right\|_{\rho+\delta}^{+} \leq\left(\frac{1}{\delta}\right)^{C(\theta) \delta^{-\frac{1}{\theta}}}
$$

and hence

$$
\|R\|_{\rho+\delta}^{+} \leq\left(\frac{1}{\delta}\right)^{C(\theta) \delta^{-\frac{1}{\theta}}}\|R\|_{\rho} .
$$

On the other hand, the coefficient of $\mathcal{M}_{a b l l^{\prime}}$ increases by at most a factor $\left(\sum_{n} a_{n}+b_{n}\right)^{2}$, then

$$
\begin{aligned}
\|R\|_{\rho+\delta} & \leq\|R\|_{\rho}^{+}\left(\sum_{n} a_{n}+b_{n}\right)^{2} e^{-\delta\left(\sum_{n}\left(2 a_{n}+k_{n}+k_{n}^{\prime}\right)|n|^{\theta}-2\left(n_{1}^{*}\right)^{\theta}\right)} \\
& \left.\leq\|R\|_{\rho}^{+}\left(2 \sum_{i \geq 3}\left(n_{i}^{*}\right)^{\theta}\right)^{2} e^{-\delta\left(2-2^{\theta}\right) \sum_{i \geq 3}\left(n_{i}^{*}\right)^{\theta}} \quad \text { (in view of (4.11) }\right) \\
& \leq \frac{16}{\left(2-2^{\theta}\right)^{2} \delta^{2}}\|R\|_{\rho}^{+}
\end{aligned}
$$

where the last inequality is based on Lemma 7.5 with $p=2$.

\section{The proof of Lemma 6.4}

\section{Proof. Step 1. The derivative of the homological equation}

Let

$$
F_{s}=\sum_{|l|=s} J^{l} \sum_{a, k, k^{\prime}} F_{s ; a k k^{\prime}}^{(l)} \mathcal{M}_{a k k^{\prime}}
$$

and let $\Psi_{s}=\left.X_{F_{s}}^{t}\right|_{t=1}$ be the time-1 map of the Hamiltonian vector field $X_{F_{s}}$. 
Using Taylor's formula,

$$
\begin{aligned}
H_{s+1}:= & \left.H_{s} \circ X_{F_{s}}^{t}\right|_{t=1} \\
= & \left.N_{*}+Z_{s}+Q_{s}\right)\left.\circ X_{F_{s}}^{t}\right|_{t=1} \\
= & N_{*}+\left\{N_{*}, F_{s}\right\}+\sum_{n \geq 2} \frac{1}{n !} \underbrace{\left\{\cdots\left\{N_{*}, F_{s}\right\}, F_{s}, \cdots, F_{s}\right\}}_{n-\text { fold }} \\
& +Q_{s s}+\sum_{n \geq 1} \frac{1}{n !} \underbrace{\left\{\cdots\left\{Q_{s s}, F_{s}\right\}, F_{s}, \cdots, F_{s}\right\}}_{n-\text { fold }} \\
& +\sum_{n \geq 0} \frac{1}{n !} \underbrace{\left\{\cdots\left\{Z_{s}+Q_{s}-Q_{s s}, F_{s}\right\}, F_{s}, \cdots, F_{s}\right\}}_{n-\text { fold }} .
\end{aligned}
$$

Now we obtain the homological equation

$$
\left\{N_{*}, F_{s}\right\}+Q_{s s}=Z_{s s},
$$

where

$$
Z_{s s}=\sum_{|l|=s} J^{l} \sum_{\substack{a, k, k^{\prime} \\ k=k^{\prime}}} Q_{s ; a k k^{\prime}}^{(l)} \mathcal{M}_{a k k^{\prime}}
$$

If the homological equation (7.39) is solvable, then we define

$$
Z_{s+1}=Z_{s}+Z_{s s}
$$

and

$$
\begin{aligned}
Q_{s+1}= & \sum_{n \geq 2} \frac{1}{n !} \underbrace{\left\{\cdots\left\{N_{*}, F_{s}\right\}, F_{s}, \cdots, F_{s}\right\}}_{n-\text { fold }} \\
& +\sum_{n \geq 1} \frac{1}{n !} \underbrace{\left\{\cdots\left\{Q_{s s}, F_{s}\right\}, F_{s}, \cdots, F_{s}\right\}}_{n-\text { fold }} \\
& +\sum_{n \geq 0} \frac{1}{n !} \underbrace{\left\{\cdots\left\{Z_{s}+Q_{s}-Q_{s s}, F_{s}\right\}, F_{s}, \cdots, F_{s}\right\}}_{n \text {-fold }},
\end{aligned}
$$

and one has

$$
H_{s+1}=N_{*}+Z_{s+1}+Q_{s+1}
$$

Step 2. The solution of the homological equation (7.39). It is easy to show that the solution of the homological equation is given by

$$
F_{s ; a k k^{\prime}}^{(l)}=\frac{Q_{s ; a k k^{\prime}}^{(l)}}{\sum_{n}\left(k_{n}-k_{n}^{\prime}\right)\left(n^{2}+\omega_{n}\right)} .
$$

In view of the fact that $\omega$ is Diophantine and following the proof of Lemma 3.2. one has

$$
\begin{aligned}
\left\|F_{s}\right\|_{\rho_{s}+\delta} & \leq C_{1}(\delta, \theta, \gamma) \cdot\left\|Q_{s s}\right\|_{\rho_{s}} \\
& \leq C_{1}(\delta, \theta, \gamma)(C(\delta, \theta, \gamma))^{(s-2) s}
\end{aligned}
$$

where the last inequality is based on (6.27) for $j=s$.

Step 3. Estimate the remainder terms $Z_{s+1}$ and $Q_{s+1}$. 
THE STABILITY OF FULL DIMENSIONAL KAM TORI FOR NONLINEAR SCHRÖDINGER EQUATION

Following the notation as (6.29), rewrite $Z_{s+1}$ as

$$
Z_{s+1}=\sum_{3 \leq j \leq s} Z_{(s+1) j}
$$

where

$$
Z_{(s+1) j}=\sum_{|l|=j} J^{l} \sum_{\substack{a, k, k^{\prime} \\ k=k^{\prime}}} Z_{s+1 ; a k k^{\prime}}^{(l)} \mathcal{M}_{a k k^{\prime}}
$$

In view of (6.29) and (7.40), one has

$$
Z_{(s+1) j}=Z_{s j}, \quad 3 \leq j \leq s .
$$

For $3 \leq j \leq s-1$, one has

$$
\begin{aligned}
\left\|Z_{(s+1) j}\right\|_{\rho_{s+1}} & =\left\|Z_{s j}\right\|_{\rho_{s+1}} \\
& \leq\left\|Z_{s j}\right\|_{\rho_{s}} \\
& \leq(C(\delta, \theta, \gamma))^{(s-2) j} \\
& \leq(C(\delta, \theta, \gamma))^{(s-1) j}
\end{aligned}
$$

In view of (7.44) and (7.45), we finish the proof of When $j=s$, one has

$$
\begin{aligned}
\left\|Z_{(s+1) s}\right\|_{\rho_{s+1}} & =\left\|Q_{s s}\right\|_{\rho_{s+1}} \\
& \leq\left\|Q_{s s}\right\|_{\rho_{s}} \\
& \leq(C(\delta, \theta, \gamma))^{(s-2) j} \\
& \leq(C(\delta, \theta, \gamma))^{(s-1) j}
\end{aligned}
$$

In view of (7.44) and (7.45), we finish the proof of (6.34).

Now we would like to estimate the norm of (7.41)-(7.42). Without loss of generality, we only consider the following term

$$
\frac{1}{n !} \underbrace{\left\{\cdots\left\{Q_{s s}, F_{s}\right\}, F_{s}, \cdots, F_{s}\right\}}_{n-\text { fold }},
$$

which is in (7.41) and contains at least $s+n(s-1) J^{\prime} s$. Then 


$$
\begin{aligned}
\|\frac{1}{n !} \underbrace{\left\{\cdots\left\{Q_{s s}, F_{s}\right\}, F_{s}, \cdots, F_{s}\right\}}_{n-\text { fold }}\|_{\rho_{s+1}} & =\|\frac{1}{n !} \underbrace{\left\{\cdots\left\{Q_{s s}, F_{s}\right\}, F_{s}, \cdots, F_{s}\right\}}_{n-\text { fold }}\|_{\rho_{s}+2 \delta} \\
& \leq \frac{1}{n !}\left(C_{2}(\delta, \theta)\left\|F_{s}\right\|_{\rho_{s}+\delta}\right)^{n}\left(\frac{n}{\delta}\right)^{n}\left\|Q_{s s}\right\|_{\rho_{s}+\delta}
\end{aligned}
$$

(following the proof of (4.22) )

$$
\leq \frac{1}{n !}\left(C_{2}(\delta, \theta) \cdot C_{1}(\delta, \theta, \gamma)\right)^{n}\left(\frac{n}{\delta}\right)^{n}\left\|Q_{s s}\right\|_{\rho_{s}}^{n+1}
$$

(in view of the first inequality in (7.43))

$$
\leq\left(\frac{e}{\delta} \cdot C_{2}(\delta, \theta) \cdot C_{1}(\delta, \theta, \gamma)\right)^{n}\left\|Q_{s s}\right\|_{\rho_{s}}^{n+1}
$$

$$
\begin{aligned}
& \left(\text { in view of } n^{n} / n ! \leq e^{n}\right) \\
\leq & (C(\delta, \theta, \gamma))^{n}\left((C(\delta, \theta, \gamma))^{(s-2) s}\right)^{n+1} \\
& (\text { in view of } \sqrt{6.20} \text { and }(\underline{6.27}) \text { for } j=s) \\
= & (C(\delta, \theta, \gamma))^{(s-2) s(n+1)+n} \\
\leq & (C(\delta, \theta, \gamma))^{(s-1)(s+n(s-1))},
\end{aligned}
$$

where the last inequality is based on

$$
(s-2) s(n+1)+n \leq(s-1)(s+n(s-1))
$$

for $s \geq 2$ and any $n \geq 0$. Hence, we finish the proof of (6.35).

\section{REFERENCES}

1. V. I. Arnold, Small denominators, 1: Mappings of the circumference onto itself, AMS Translations, 46 (1965), 213-288 (Russian original published in 1961).

2. P. Baldi, M. Berti and R. Montalto, KAM for quasi-linear and fully nonlinear forced perturbations of Airy equation, Math. Ann. 359 (2014), no. 1-2, 471-536.

3. P. Baldi, M. Berti and R. Montalto, KAM for autonomous quasi-linear perturbations of KdV, Ann. Inst. H. Poincare Anal. Non Lineaire, 33 (2016), no. 6, 1589-1638.

4. D. Bambusi and A. Giorgilli, Exponential stability of states close to resonance in infinitedimensional Hamiltonian systems, J. Statist. Phys. 71 (1993), no. 3-4, 569-606.

5. D. Bambusi, Birkhoff normal form for some nonlinear PDEs, Comm. Math. Phys. 234 (2003), no. 2, 253-285.

6. D. Bambusi and B. Grébert, Birkhoff normal form for partial differential equations with tame modulus Duke Math. J. 135 (2006), no. 3, 507-567.

7. D. Bambusi, J. M. Delort, B. Grébert and J. Szeftel, Almost global existence for Hamiltonian semilinear Klein-Gordon equations with small Cauchy data on Zoll manifolds, Comm. Pure Appl. Math. 60 (2007), no. 11, 1665-1690.

8. D. Bambusi, A Birkhoff normal form theorem for some semilinear PDEs, Hamiltonian dynamical systems and applications. (2008), 213-247.

9. D. Bambusi and N. N. Nekhoroshev, A property of exponential stability in nonlinear wave equations near the fundamental linear mode, Phys. D. 122 (1998), no. 1-4, 73-104.

10. G. Benettin, J. Fröhlich and A. Giorgili, A Nekhoroshev-type theorem for Hamiltonian systems with infinitely many degrees of freedom, Comm. Math. Phys. 119 (1988), no. 1, 95-108.

11. M. Berti and L. Biasco, Branching of Cantor manifolds of elliptic tori and applications to PDEs, Comm. Math. Phys., 305 (2011), no. 3, 741-796.

12. J. Bourgain, Construction of approximative and almost periodic solutions of perturbed linear Schrödinger and wave equations, Geom. Funct. Anal. 6 (1996), no. 2, 201-230.

13. J. Bourgain, On Melnikov's persistence problem, Math. Res. Lett. 4 (1997), no. 4, 445-458. 
THE STABILITY OF FULL DIMENSIONAL KAM TORI FOR NONLINEAR SCHRÖDINGER EQUATIOA9

14. J. Bourgain, Quasi-periodic solutions of Hamiltonian perturbations of $2 D$ linear Schrödinger equation, Ann. of Math. 148 (1998), no. 2, 363-439.

15. J. Bourgain, On diffusion in high-dimensional Hamiltonian systems and PDE, J. Anal. Math. 80 (2000), 1-35.

16. J. Bourgain, Remark on stability and diffusion in high-dimensional Hamiltonian systems and partial differential equations, Ergodic Theory Dynam. Systems 24 (2004), no. 5, 1331-1357.

17. J. Bourgain, Recent progress on quasi-periodic lattice Schrödinger operators and Hamiltonian PDEs Russian Math. Surveys 59:2 (2004), 231-246.

18. J. Bourgain, Green function estimates for lattice Schrödinger operators and applications, Annals of Mathematics Studies, Princeton University Press (2005).

19. J. Bourgain, On invariant tori of full dimension for $1 D$ periodic $N L S$, J. Funct. Anal., 229 (2005), no. 1, 62-94.

20. H. Cong, J. Liu and X. Yuan, Stability of KAM tori for nonlinear Schrödinger equation, Mem. Amer. Math. Soc, 239 (2016), no. 1134.

21. H. Cong, M. Gao and J. Liu, Long time stability of KAM tori for nonlinear wave equation, J. Differential Equations, 258 (2015), no. 8, 2823-2846.

22. W. Craig. and C. Wayne. Newton's method and periodic solutions of nonlinear wave equation, Comm. Pure. Appl. Math. 46 (1993), no. 11, 1409-1498.

23. J. M. Delort, Long-time Sobolev stability for small solutions of quasi-linear Klein-Gordon equations on the circle, Trans. Amer. Math. Soc. 361 (2009), no. 8, 4299-4365.

24. J. M. Delort and J. Szeftel, Long-time existence for small data nonlinear Klein-Gordon equations on tori and spheres, Int. Math. Res. Not. 37 (2004), no. 37, 1897-1966.

25. L. H. Eliasson and S. B. Kuksin KAM for the nonlinear Schrödinger equation, Ann. of Math. 172 (2010), no. 1, 371-435.

26. L. H. Eliasson, Perturbations of stable invariant tori for Hamiltonian systems, Ann. Scuola Norm. Sup. Pisa Cl. Sci. 15 (1988), no. 1, 115-147.

27. E. Faou and B. Grébert, A Nekhoroshev-type theorem for the nonlinear Schrödinger equation on the torus Anal. PDE. 6 (2013), no. 6, 1243-1262.

28. R. Feola and M. Procesi, Quasi-periodic solutions for fully nonlinear forced reversible Schrodinger equations, J. Differential Equations. 259 (2015), no. 7, 3389-3447.

29. B. Grébert, R. Imekraz and É. Paturel, Normal forms for semilinear quantum harmonic oscillators Comm. Math. Phys. 291 (2009), no. 3, 763-798.

30. A. N. Kolmogorov, On the conservation of conditionally periodic motions under small perturbations of the hamiltonian, Dokl. Akad. Nauk, SSSR, 98 (1954), 527-530.

31. S. B. Kuksin, Hamiltonian perturbations of infinite-dimensional linear systems with an imaginary spectrum, Funct. Anal. Appl. 21 (1987), 192-205.

32. S. B. Kuksin, Nearly integrable infinite-dimensional Hamiltonian systems, Springer-Verlag, Berlin 1993.

33. S. B. Kuksin and J. Pöschel, Invariant Cantor manifolds of quasi-periodic oscillations for a nonlinear Schrödinger equation, Ann. of Math. 143 (1996), no. 1, 149-179.

34. S. B. Kuksin, Analysis of Hamiltonian PDEs, Oxford Univ. Press, Oxford, 2000

35. S. B. Kuksin, Fifteen Years of KAM for PDE geometry, topology, and mathematical physics, Amer. Math. Soc. Transl. 2: 212 (2004), 237-258.

36. J. Liu and X. Yuan, A KAM Theorem for Hamiltonian Partial Differential Equations with Unbounded Perturbations, Commun. Math. Phys., 307 (2011), no. 3, 629-673.

37. A. Morbidelli and A. Giorgilli, Superexponential Stability of KAM Tori, J. Statist. Phys. 78 (1995), no. 5-6, 1607-1617.

38. J. Moser, On invariant curves of area-preserving mappings of an annulus, Nachr. Akad. Wiss. Göttingen, Math. Phys. Kl, (1962), 1-20.

39. N. Nekhoroshev, An exponential estimate of the time of stability of nearly-integrable Hamiltonian systems, (Russian) Uspehi Mat. Nauk 32 (1977), no. 6, 5C66.

40. T. Kappeler and J. Pöschel, KdVEGKAM, Springer-Verlag, Berlin Heidelberg, 2003

41. J. Pöschel, Small divisors with spatial structure in infinite dimensional Hamiltonian systems, Comm. Math. Phys. 127 (1990), no. 2, 351-393.

42. J. Pöschel, On Nekhoroshev's Estimate at an Elliptic Equilibrium, Internat. Math. Res. Notices 4 (1999), no. 4, 203C215.

43. J. Pöschel, On the construction of almost periodic solutions for nonlinear Schrödinger equations, Ergodic Theory Dynam. Systems, 5 (2002), no. 5, 1537-1549. 
44. C. E. Wayne, Periodic and quasi-periodic solutions of nonlinear wave equations via KAM theory, Comm. Math. Phys. 127 (1990), no. 3, 479-528.

45. X. Yuan and J. Zhang, Long time stability of Hamiltonian partial differential equations, SIAM J. Math. Anal. 46 (2014), no. 5, 3176-3222.

46. J. Zhang, M. Gao and X. Yuan, KAM tori for reversible partial differential equations, Nonlinearity 24 (2011), no. 4, 1198-1228.

School of Mathematical Sciences, Dalian University of Technology, Dalian, LiaonING 116024, CHINA

E-mail address: conghongzi@dlut.edu.cn

School of Mathematical Sciences, Sichuan University, Chengdu, Sichuan 610065, CHINA

E-mail address: liujj@fudan.edu.cn

School of Mathematical Sciences, Fudan University, Shanghai 200433, China

E-mail address: yunfengshi13@fudan.edu.cn

School of Mathematical Sciences, Fudan University, Shanghai 200433, China

E-mail address: xpyuan@fudan.edu.cn 